
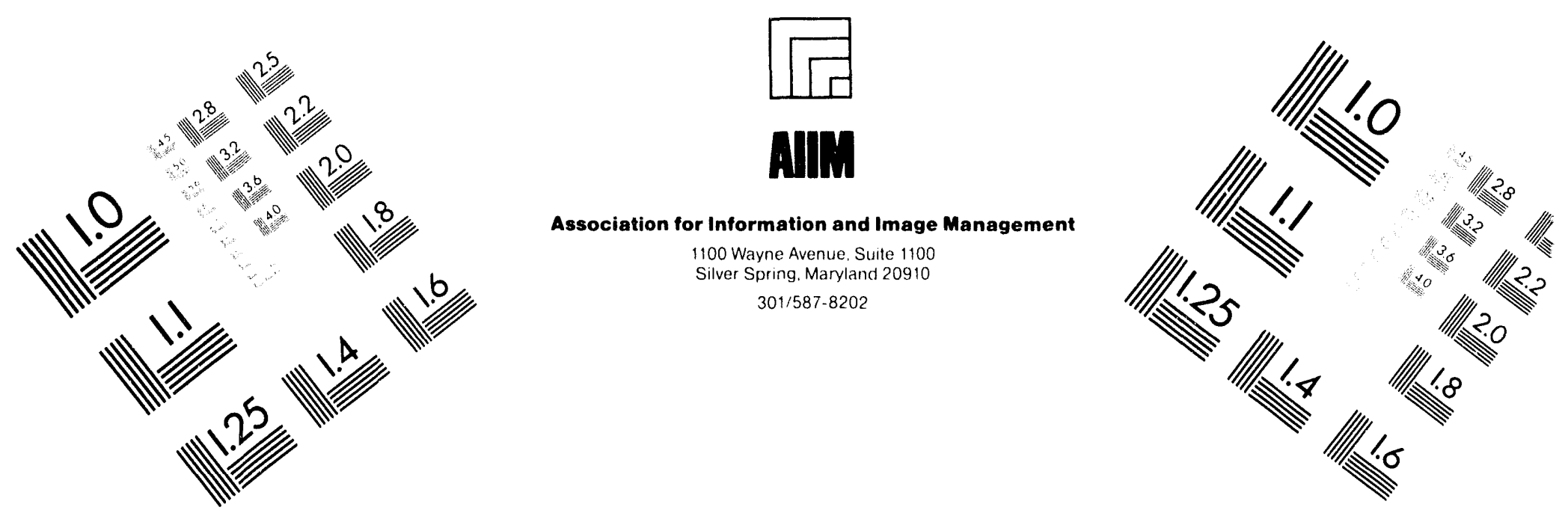

\title{
Centimeter
}

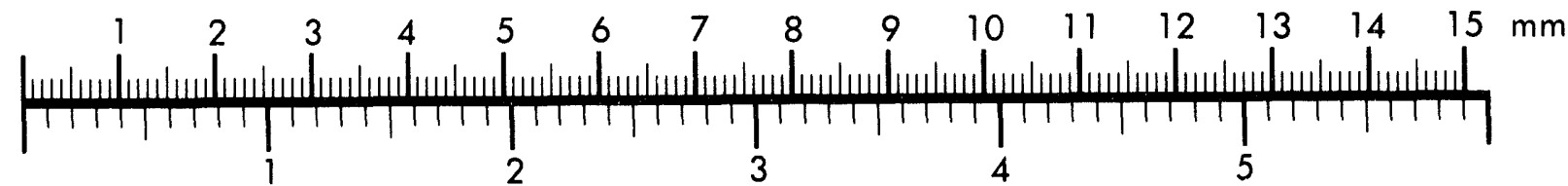
Inches
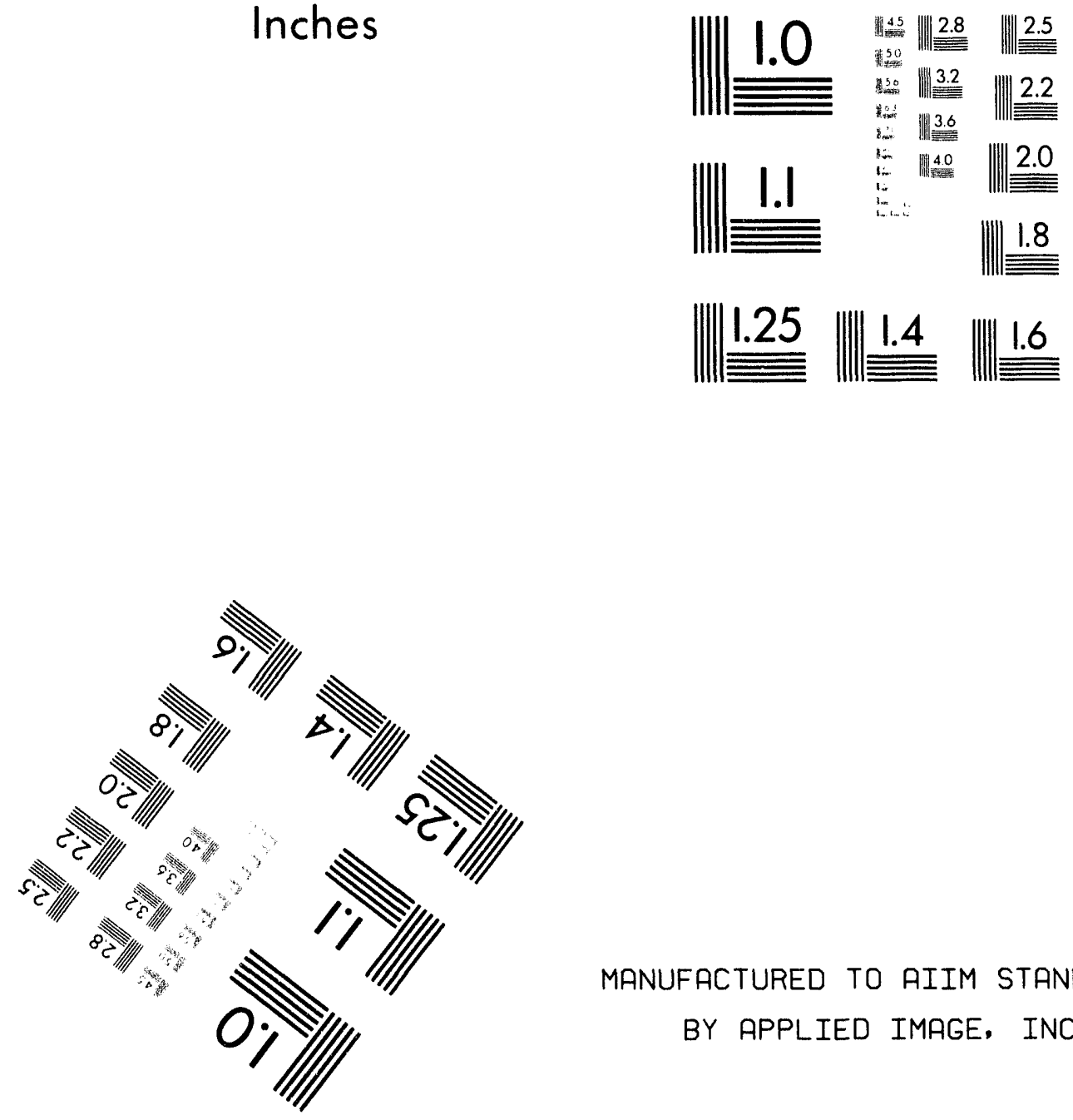

MANUFACTURED TO AIIM STANDARDS

BY APPLIED IMAGE, INC.

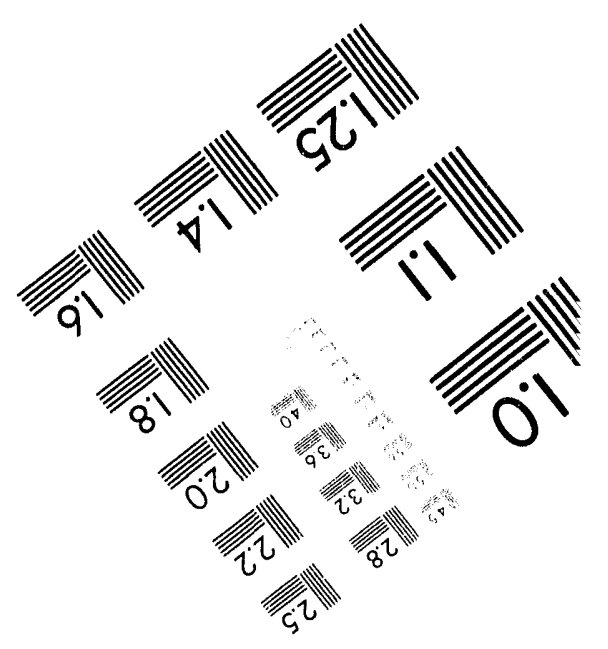



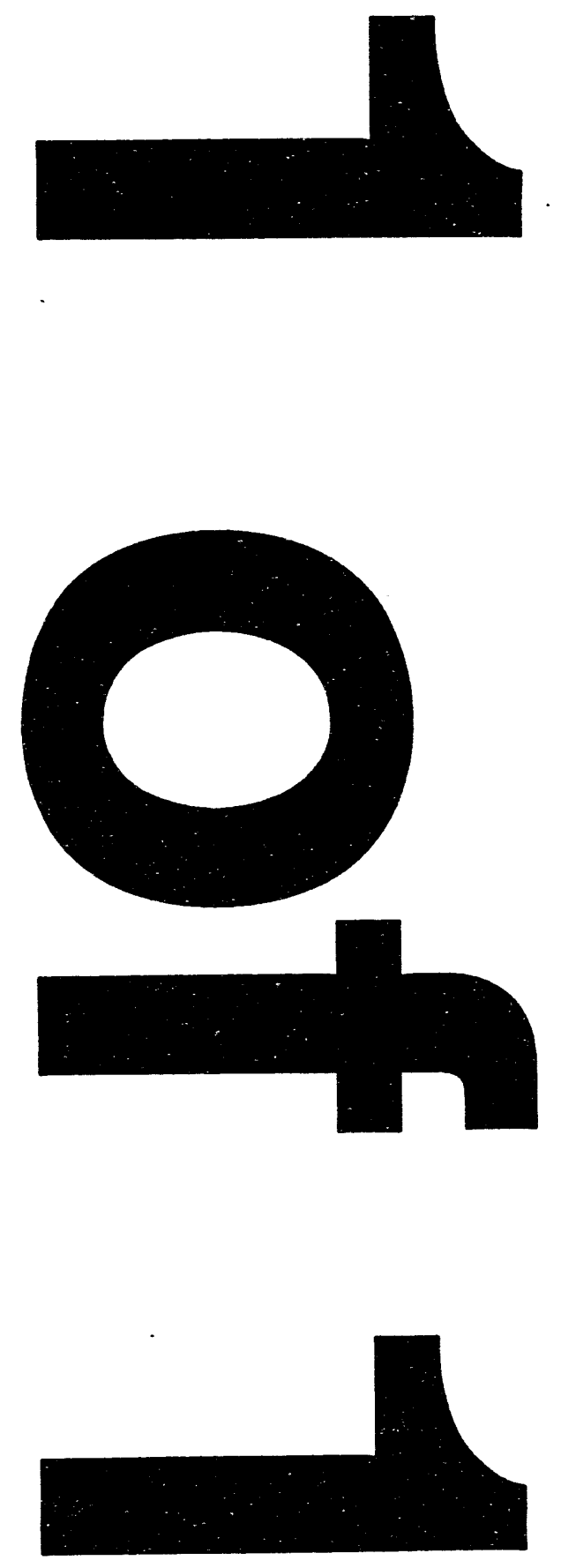


\section{BENCH-SCALE TESTING OF THE MULTI-GRAVITY SEPARATOR IN COMBINATION WITH MICROCEL}

Contract No: DE-AC22-92PC92205

Fifth Quarterly Report

Reporting Period:

October 1, 1993 to December 31, 1993

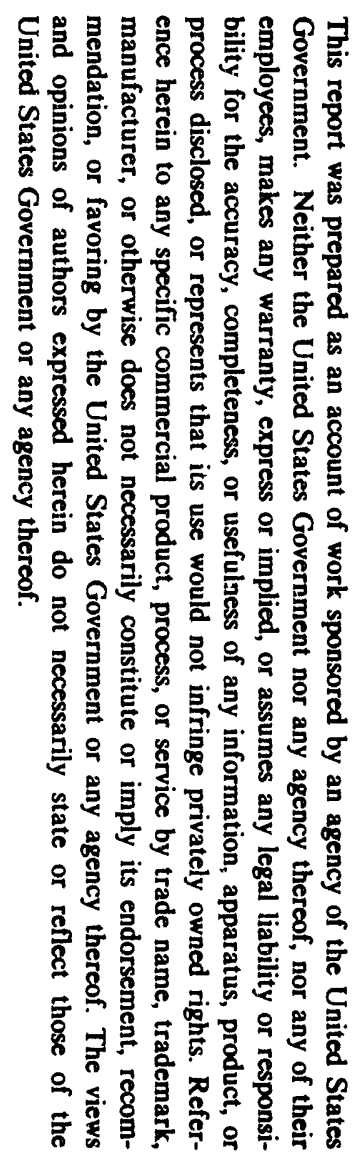

\section{Prime Contractor:}

Center for Coal and Minerals Processing Virginia Polytechnic Institute and State University Blacksburg, Virginia 24061-0258

\section{Subcontractors:}

Carpco, Inc.

Roberts \& Schaefer Company

Consolidation Coal Company

Kerr-McGee Coal Corporation

\section{Prepared for:}

Pittsburgh Energy Technology Center

U.S. Department of Energy

P.O. Box 10940

Pittsburgh, Pennsylvania 15236

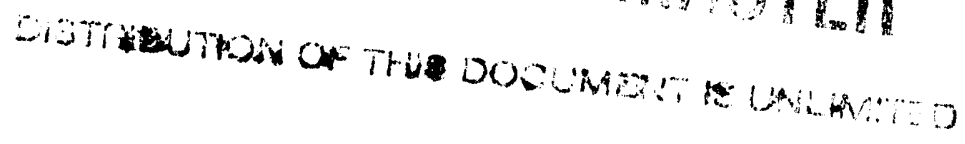

Contracting Officer's Representative: Carl Maronde

U.S./DOE Patent Clearance is not required prior to the publication of this document. 


\section{TABLE OF CONTENTS}

TABLE OF CONTENTS $\ldots \ldots \ldots \ldots \ldots \ldots \ldots \ldots \ldots$

1.0 Introduction and Summary $\ldots \ldots \ldots \ldots \ldots \ldots$

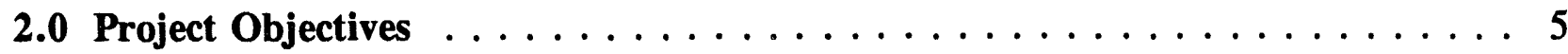

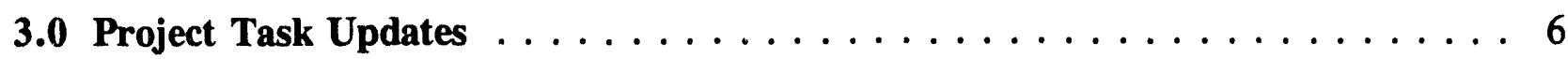

3.1 Task $1.0-$ Project Planning $\ldots \ldots \ldots \ldots$

3.2 Task 4.0 -Sample Characterization $\ldots \ldots \ldots \ldots$

Subtask 4.1 - Preliminary Characterization ............... 6

Subtask 4.2 - Washability Characterization ............... 6

Subtask 4.3 - Release Analysis Characterization . . . . . . . . . . . 6

3.3 Task $9.0-$ MGS Scale-Up $\ldots \ldots \ldots \ldots$

Subtask 9.1 - Development of Scale-Up Criteria . . . . . . . . . . . . 6

Subtask 9.2 - Scale-Up Validation . . . . . . . . . . . . . . . 7

3.4 Task 10.0 - Detailed Testing $\ldots \ldots \ldots \ldots \ldots \ldots$

Subtask 10.1 - Microcel Testing (Independent) . . . . . . . . . . 8

Subtask 10.2 - MGS Testing (Independent) . . . . . . . . . . . . 8

Subtask 10.3 - Combined Microcel and MGS Testing . . . . . . . . . 9

Subtask 10.4 - Combined Microcel/MGS/WOC Testing . . . . . . . . 10

Subtask 10.5 - Long Duration Testing . . . . . . . . . . . . . . . . 10

3.5 Task 11.0 - Decommissioning $\ldots \ldots \ldots \ldots$

3.6 Task 12.0 -Sample Analysis $\ldots \ldots \ldots \ldots \ldots$

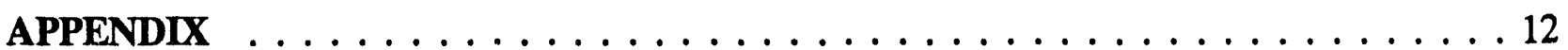

Tables PITT-1 to PITT-22 and ILL-1 to ILL-22 . . . . . . . . . . . . . . 12

Figures PITT-1 to PITT-8 and ILL-1 to ILL-8 . . . . . . . . . . . . . . . 12 


\section{LIST OF FIGURES}

Table 1. Status of major project tasks as of December $31,1993 \ldots \ldots \ldots$

Table 2. Test numbering system $\ldots \ldots \ldots \ldots \ldots \ldots \ldots \ldots \ldots \ldots \ldots \ldots \ldots \ldots \ldots$ 


\subsection{Introduction and Summary}

During the quarter ending December, 31, 1993, the independent, combined and long duration testing were completed for both the Pittsburgh \#8 coal and the Illinois \#6 coal. Overall, the project is on schedule and the bulk of the critical work, from a timing perspective, is complete. Table 1 summarizes the status of major project tasks as of December 31, 1993.

Preliminary results provide strong evidence that combining the Microcel flotation column with the Multi-Gravity Separator has a synergistic effect. Overall ash and pyritic sulfur rejections of $75 \%$, at a $90 \%$ combustible recovery, were consistently achieved on the Pittsburgh \#8 seam coal. On the Illinois \#6 coal, pyritic sulfur rejections over $75 \%$ and combustible recoveries of over $85 \%$ were achieved. These results are discussed in this report. Although further analysis is taking place, it is very evident from the results presented herein that a well-designed and -operated flotation column performs well for ash rejection but not as well for pyrite rejection. It is equally evident that a good fine gravity separator can reject pyrite from coal but perform more poorly for ash rejection. The concept of combining the best of both units into one circuit has therefore been successfully tested in this project.

Table 1. Status of major project tasks as of December 31, 1993.

\begin{tabular}{||l|l||}
\hline \multicolumn{1}{|c|}{ MAJOR TASKS } & \multicolumn{1}{|c|}{ STATUS } \\
\hline \hline Task 1 - Project Planning & Ongoing \\
Task 2 - CPPRF Modifications & Complete \\
Task 3 - Sample Acquisition & Complete \\
Task 4 - Sample Characterization & Ongoing \\
Task 5 - Flowsheet Development and Engineering Design & Complete \\
Task 6 - Procurement and Fabrication & Complete \\
Task 7 - Process Module Installation & Complete \\
Task 8 - Shakedown Testing & Complete \\
Task 9 - MGS Scale-Up & Ongoing \\
Task 10 - Detailed Testing & Ongoing \\
Task 11 - Decommissioning & Planned \\
Task 12 - Sample Analysis & Ongoing \\
Task 13 - Final Report & Planned \\
\hline
\end{tabular}




\subsection{Project Objectives}

The primary objective of the proposed work is to design, install, and operate an advanced fine coal processing circuit combining the Microcel $^{\mathrm{TM}}$ and Multi-Gravity Separator (MGS) technologies. Both of these processes have specific advantages as stand-alone units. For example, the Microcel column effectively removes ash-bearing mineral matter, while the MGS efficiently removes coal-pyrite composites. By combining both unit operations into a single processing circuit, synergistic advantages can be gained. As a result, this circuit arrangement has the potential to improve coal quality beyond that achieved using the individual technologies.

In addition to the primary objective, secondary objectives of the proposed test program include:

- Circuit Optimization: The performance of each unit operation, individually and combined, will be optimized by conducting parametric studies as a function of key operating variables. The goal of this work is to maximize the rejections of pyritic sulfur and ash while maintaining a high energy recovery.

- Process Variability: The steady-state performance of the optimized processing circuit will be studied (i) by conducting several long-duration test runs over a period of several days and (ii) by testing coal samples from other sources specified by the participating coal companies.

- Process Evaluation: Detailed technical and economic evaluations will be conducted to examine the feasibility of the proposed concept for fine coal cleaning on an industrial scale. This evaluation will include a projected cost-benefit analysis and a review of all test data, engineering analyses, scale-up procedures, and process deficiencies.

The test work is being conducted at the Pittsburgh Energy Technology Center's Coal Preparation Process Research Facility (CPPRF) located in Pittsburgh, Pennsylvania. The CPPRF is a state-of-the-art pilot-scale facility for coal preparation research and testing. The Emerging Technology (ET) section of the pilot plant is being used for testing the combined Microcel and MGS circuit. The ET area, and subsequently installed mezzanine, is adjacent to the pilot plant and was established for testing new and emerging technologies in coal preparation. This facility is ideally suited for pilot-scale test work due to the availability of all necessary ancillary facilities (i.e., bulk solids handling, preparation, and waste disposal). In addition, the necessary environmental, safety and health aspects related to the handling and disposal of waste are already in place. 


\subsection{Project Task Updates}

Those tasks shown as completed in previous quarterly reports will not be mentioned herein (i.e. Tasks $2,3,5,6,7,8$ ).

\subsection{Task 1.0 - Project Planning}

All project planning is complete and with this report all reporting will be current. All required plans and reports have been submitted. Topical reports on sample characterization and on the ET Area Modification are forthcoming.

\subsection{Task 4.0 - Sample Characterization}

\section{Subtask 4.1 - Preliminary Characterization}

Utilizing a hammermill, -28 mesh and -65 mesh samples were produced independently. The size-by-size sulfur and ash analyses for each of the two seams and each of the two feed sizes were given in the Second Quarterly Report.

\section{Subtask 4.2 - Washability Characterization}

Since all efforts were concentrated on the detailed testing at the CPPRF, no washability type characterizations were performed this quarter. As discussed in the Fourth Quarterly Report, initial high-G centrifuge washability results were not satisfactory. Further washability characterizations will include high-G centrifuge separations by an outside group skilled in such work.

\section{Subtask 4.3 - Release Analysis Characterization}

Release analysis results for both coals were presented in the Second Quarterly Report and will be included in the Preliminary Characterization Topical Report along with a complete summary of all the characterization data..

\subsection{Task 9.0 - MGS Scale-Up}

\section{Subtask 9.1 - Development of Scale-Up Criteria}

Development of a process model for the MGS requires identification of those operating parameters which affect performance. Detailed testing results will be used to identify these relevant operating parameters which will be applied to the scale-up in future quarters. 


\section{Subtask 9.2 - Scale-Up Validation}

After completion of the detailed testing, the criteria required for scale-up modeling will be reviewed and quantified to the extent possible.

\subsection{Task 10.0 - Detailed Testing}

Tests using the Microcel flotation column and the Multi-Gravity Separator (MGS) unit were conducted with independent raw coal feeds. After the major control parameters were determined, testing began on the combined circuit in which the column product reported to the MGS as feed. The optimum operating settings were then used for a long duration run of the combined circuit. The entire process was then repeated for the second coal.

Detailed testing by subtask is described below. Although the chronological sequence was to complete the independent unit testing, the combined units testing and long duration testing on the Pittsburgh \#8 coal before proceeding to the identical sequence of unit testing on the Illinois \#6 coai. This section gives results based on sample analysis for ash, total sulfur, and pyritic sulfur. Due to the excellent correlation of ash and BTU, the ash analyses were utilized for performance decisions in lieu of BTU. This allowed the BTU analyses to be delayed in order to keep the project on schedule. BTU results will be included in the final report. A systematic approach was used in assigning test numbers as shown in the following table.

Table 2. Test numbering system.

\begin{tabular}{||l|l|l||}
\hline \multicolumn{1}{|c|}{$\begin{array}{c}\text { TEST NUMBER } \\
\text { RANGES }\end{array}$} & \multicolumn{1}{c|}{ CIRCUIT } & \multicolumn{1}{c|}{ COAL SEAM } \\
\hline $101-199$ & Microcel Only & Pittsburgh \#8 \\
\hline $201-299$ & MGS Only & Pittsburgh \#8 \\
\hline $301-399$ & Microcel Conc. to MGS & Pittsburgh \#8 \\
\hline PITT-LD & Microcel Conc. to MGS & Pittsburgh \#8 \\
\hline $401-499$ & Microcel Only & Illinois \#6 \\
\hline $501-599$ & MGS Only & Illinois \#6 \\
\hline $601-699$ & Microcel Conc. to MGS & Illinois \#6 \\
\hline ILL-LD & Microcel Conc. to MGS & Illinois \#6 \\
\hline
\end{tabular}




\section{Subtask 10.1 - Microcel Testing (Independent)}

\section{Pittsburgh \#8 Coal}

The Microcel test matrix for the Pittsburgh $\# 8$ coal, Table PITT-1 of the Appendix, consisted of a three-level Box-Behnken test matrix containing 3 feed sizes, 3 feed rates, and 3 frother dosages. This resulted in 15 tests with 2 more added, to provide a check on repeatability for a total of 17 tests in this series. Table PITT-2 gives the analyses of test numbers $101-117$, while Microcel performance results are found in Table PITT-3. The performance in terms of ash, sulfur, and pyritic rejection versus combustible recovery is shown in Figure PITT-1.

\section{Illinois \#6 Coal}

The Microcel test matrix for the Illinois \#6 coal, Table ILL-1, involved 9 tests in which collector and frother dosages were varied. The 3 settings for each included a low, medium, and high dosage. Analyses of tests numbers 401 - 409 are given in Table ILL-2, while the performance results are found in Table ILL-3. Figure ILL-1 displays the performance in terms of ash, sulfur, and pyritic rejection versus combustible recovery. Results of all 9 tests were very similar with the optimums having combustible recoveries of 86 to $88 \%$, ash rejections in the low 80's and pyritic sulfur rejections in the low 60 's.

\section{Subtask 10.2 - MGS Testing (Independent)}

\section{Pittsburgh \#8 Coal}

Raw Pittsburgh \#8 coal was used as feed for independent MGS tests. This series of tests utilized a 5-level test matrix (Table PITT-4) which included grind size, drum speed, wash water, percent solids feed, and feed rate as variables. Testing these five variables over three ranges yielded 43 test results, test numbers 201-243. Tables PITT-5 to PITT-8 give the analytical results while Figure PITT-2 graphically illustrates the ash, sulfur, and pyritic rejections versus combustible recovery. Comparing Figure PITT-2 to PITT-1 shows that even over a broad range of variables the Microcel is clearly best at ash rejection and the MGS performs best at pyrite rejection.

\section{Illinois \#6 Coal}

The MGS testing involved a 4 level test matrix in which drum speed, wash water, percent solids in feed, and feed rate were varied. As before, each parameter was varied over 3 ranges: low, medium and high. As a result, 27 tests were conducted and assigned tests numbers 501-527. The MGS constant and variable conditions are listed in Table ILL-4.

Results of the analyses are given in Table ILL-5 to ILL-7. Performance data are found in Table ILL-8 while Figure ILL-2 details graphically the rejections versus 
combustible recovery for the MGS unit operating on raw Illinois \#6 feed. A comparison of Figure ILL-2 with Figure ILL-1 illustrates that, even with many parameters being adjusted over a broad range, the Microcel excels at ash rejection whereas the MGS rejects pyrite much better than ash. Ash rejection for the MGS is noticeably lower than the column throughout the range of settings.

\section{Subtask 10.3 - Combined Microcel and MGS Testing}

\section{Pittsburgh \#8 Coal}

Combined Microcel and MGS testing allowed the Microcel column product to feed the MGS directly via a holding sump. The intermediate sump absorbed any possible surges as well as allowing the MGS feed rate to be independently controlled. The well-mixed sump ran with a constant overflow which ensured a constant feed rate to the MGS. A 4-level test matrix with a low, medium and high value for each variable was also used for this series of tests. Column feed rate was the only variable used for the Microcel column while the MGS variables were drum speed, wash water, and MGS feed rate. These conditions are listed in Table PITT-9 for test numbers 301-327.

Samples were taken independently around the Microcel and MGS, which allowed for complete evaluation of each unit as well as the total circuit. Analyses and results are found in Tables PITT-10 through PITT-15. Ash, sulfur, and pyritic rejections versus combustible recoveries for the Microcel are graphed in Figure PITT-3. The MGS and combined circuit graphs are in Figures PITT-4 and PITT-5, respectively. Once again the Microcel plot shows that it is best at ash rejection while the MGS plot shows advantages in pyrite rejection. The combined results plotted in Figure PITT-5, however, show that the best of both can be obtained with the combination of Microcel and MGS. The synergy of combining the two units is well illustrated when comparing Figures PITT-3, -4 , and -5 . The excellent ash and pyrite rejections are obtained while maintaining a high recovery. It is important to remember when viewing these plots, that the scatter in the data is due to intentionally varying several control parameters. Even with these variations, the performance remains within relatively close patterns.

\section{Illinois \#6 Coal}

Microcel conditions were held constant for the combined testing of the Illinois \#6 coal. All four variables of the 4-level matrix were applied to the MGS. As listed in Table ILL-9, these were drum speed, wash water, shake amplitude, and MGS feed rate.

Results of this series of tests are found in Tables ILL-10 through ILL-15. The ash, sulfur, and pyrite rejections versus combustible recoveries for the Microcel are shown graphically in Figure ILL-3. The MGS results are graphed in Figure ILL-4 and the combined circuit results are shown in Figure ILL-5. As with the Pittsburgh \#8 seam, the Illinois \#6 results show that the combination of Microcel flotation and MGS provide the best of both for rejection of ash and pyrite. 


\section{Subtask 10.4 - Combined Microcel/MGS/WOC Testing}

Three tests were conducted with the combined circuit including a small water-only cyclone. The Microcel concentrate was pumped to the cyclone from which the overflow was taken as a final product and the underflow was fed to the MGS for pyrite removal. The object of this test series was to reduce the loading on the MGS unit. If successful, this would increase the capacity of the total circuit for a given MGS size. This testing was performed just prior to the end of the quarter and the results will be detailed in subsequent reports.

\section{Subtask 10.5 - Long Duration Testing}

\section{Pittsburgh \#8 Coal}

From the results of the combined testing the best tests were identified based on ash and pyrite rejections. The operating parameters used in those tests provided the basis for the selection of operating parameters to be used for the long duration testing. Long duration testing involved holding all control parameters constant for a continuous run of over 10 hours. These operating parameters are given in Table PITT-16.

The results as listed in Tables PITT-17 to PITT-22 indicate the consistency with which the system performed. Figures PITT-6 to PITT-8 show graphically the ash, sulfur, and pyrite rejection for the Microcel, MGS, and combined circuit. As before, the synergism resulted in the best of both units being brought together. For the combined circuit, ash and pyrite rejections of over $75 \%$ were obtained at a $90 \%$ combustibles recovery.

\section{Illinois \#6 Coal}

As discussed above, the best results of the combined testing were used to select the operating parameters to be held constant during the long duration testing of the Illinois \#6 coal. The parameters given in Table ILL-16 produced the results listed in Tables ILL-17 to ILL-22. Figures ILL-6 to ILL-8 show graphically the ash, sulfur, and pyrite rejection for the Microcel, MGS, and combined circuit. The synergistic effect is again evident in the performance graphs. For the overall circuit, ash and pyrite rejections of over $75 \%$ were obtained at a combustibles recovery of over $85 \%$. Although the Illinois \#6 had a much higher feed ash than the Pittsburgh \#8 coal, and was definitely more difficult to clean, the results were very close to those of the Pittsburgh \#8 coal.

\subsection{Task 11.0 - Decommissioning}

Decommissioning will begin during the middle of January, 1994, or as soon as the near-term testing is complete, and will last no more than three weeks. As stated in the Decommissioning Plan, all equipment added during this project will be removed and the CPPRF will be restored to the condition found prior to the beginning of this project. 


\subsection{Task 12.0 - Sample Analysis}

Sample analysis is being conducted as required on the detailed testing. Samples are being analyzed initially for \% solids, ash, total sulfur, and pyritic sulfur. Selected samples will be further analyzed for heating value, sizing, and washability analysis. 


\section{APPENDIX}

Tables PITT-1 to PITT-22 and ILL-1 to ILL-22

Figures PITT-1 to PITT-8 and ILL-1 to ILL-8 
Microcel/MGS Independent Testing - Pittsburgh \#8

Microcel Conditions Held Constant

Collector Dosage (Ibs/ton):

Wash Water (Ipm):

Percent Solids (W/W):

Air Flow Rate (SCFM):

\section{1}

40.13

11-12

9.01

\section{Microcel Variables}

\begin{tabular}{|c|c|c|c|}
\hline $\begin{array}{c}\text { Test } \\
\text { Number }\end{array}$ & $\begin{array}{c}\text { Feed } \\
\text { Size } \\
\text { (microns) } \\
\end{array}$ & $\begin{array}{c}\text { Feed } \\
\text { Rate } \\
\text { (lbs/hr) }\end{array}$ & $\begin{array}{l}\text { Frother } \\
\text { Dosage } \\
\text { (ml/min) }\end{array}$ \\
\hline & & & \\
\hline 101 & 300 & 300 & 1.00 \\
\hline 102 & 300 & 400 & 0.50 \\
\hline 103 & 300 & 500 & 1.00 \\
\hline 104 & 300 & 400 & 1.50 \\
\hline 105 & 200 & 400 & 1.00 \\
\hline 106 & 200 & 500 & 0.50 \\
\hline 107 & 200 & 500 & 1.50 \\
\hline 108 & 200 & 400 & 1.00 \\
\hline 109 & 100 & 300 & 1.00 \\
\hline 110 & 100 & 400 & 0.50 \\
\hline 111 & 100 & 500 & 1.00 \\
\hline 113 & 200 & 300 & 1.50 \\
\hline 114 & 200 & 400 & 1.00 \\
\hline 116 & 200 & 300 & 1.50 \\
\hline 117 & 200 & 400 & 1.00 \\
\hline
\end{tabular}


Microcel/MGS Independent Tests Results - Pittsburgh \#8

Microcel - Ash

\begin{tabular}{|c|c|c|c|c|c|c|c|c|}
\hline $\begin{array}{c}\text { Test } \\
\text { Number }\end{array}$ & $\begin{array}{l}\text { Moasured } \\
\text { Column } \\
\text { Feed } \\
\text { Ash } \\
\%\end{array}$ & $\begin{array}{l}\text { Adjusted } \\
\text { Column } \\
\text { Feed } \\
\text { Ash } \\
\% \\
\end{array}$ & $\begin{array}{c}\text { Measured } \\
\text { Column } \\
\text { Product } \\
\text { Ash } \\
\%\end{array}$ & $\begin{array}{c}\text { Adjusted } \\
\text { Column } \\
\text { Product } \\
\text { Ash } \\
\% \\
\end{array}$ & $\begin{array}{c}\text { Moasured } \\
\text { Column } \\
\text { Reject } \\
\text { Ash } \\
\%\end{array}$ & $\begin{array}{c}\text { Adjusted } \\
\text { Column } \\
\text { Reject } \\
\text { Ash } \\
\%\end{array}$ & $\begin{array}{l}\text { Measured } \\
\text { Column } \\
\text { Ash } \\
\text { Yield } \\
\%\end{array}$ & $\begin{array}{l}\text { Adjusted } \\
\text { Column } \\
\text { Ash } \\
\text { Yield } \\
\% \\
\end{array}$ \\
\hline & & & & & & & & \\
\hline 101 & 20.67 & 20.67 & 12.84 & 12.84 & 78.25 & 78.25 & 88.03 & 88.03 \\
\hline 102 & 18.57 & 18.57 & 7.63 & 7.63 & 63.42 & 63.42 & 80.39 & 80.39 \\
\hline 103 & 21.25 & 21.25 & 8.97 & 8.97 & 74.65 & 74.65 & 81.30 & 81.30 \\
\hline 104 & 21.45 & 21.45 & 11.97 & 11.97 & 80.10 & 80.10 & 86.09 & 86.09 \\
\hline 105 & 20.30 & 20.30 & 9.14 & 9.14 & 78.77 & 78.77 & 83.97 & 83.97 \\
\hline 106 & 19.37 & 19.40 & 6.59 & 6.59 & 45.74 & 45.69 & 67.36 & 67.24 \\
\hline 107 & 19.57 & 19.57 & 9.31 & 9.31 & 76.06 & 76.06 & 84.63 & 84.63 \\
\hline 108 & 19.64 & 19.64 & 9.30 & 9.30 & 78.29 & 78.29 & 85.01 & 85.01 \\
\hline 109 & 19.82 & 19.82 & 10.16 & 10.16 & 72.89 & 72.89 & 84.60 & 84.60 \\
\hline 110 & 20.60 & 20.60 & 10.68 & 10.68 & 75.33 & 75.33 & 84.66 & 84.66 \\
\hline 111 & 20.66 & 20.65 & 11.04 & 11.04 & 75.50 & 75.52 & 85.08 & 85.10 \\
\hline 113 & 20.99 & 20.99 & 18.32 & 18.32 & 77.73 & 77.73 & 95.51 & 95.51 \\
\hline 114 & 20.62 & 20.70 & 9.58 & 9.57 & 68.75 & 68.59 & 81.34 & 81.14 \\
\hline 116 & 18.94 & 18.94 & 11.24 & 11.24 & 76.98 & 76.98 & 88.29 & 88.29 \\
\hline 117 & 14.19 & 14.23 & 7.03 & 7.02 & 64.47 & 64.36 & 87.53 & 87.43 \\
\hline
\end{tabular}

Microcel - Total Sulfur

\begin{tabular}{|c|c|c|c|c|c|c|}
\hline $\begin{array}{c}\text { Test } \\
\text { Number }\end{array}$ & $\begin{array}{c}\text { Measured } \\
\text { Column } \\
\text { Feed } \\
\text { T. Sulfur } \\
\%\end{array}$ & $\begin{array}{l}\text { Adjusted } \\
\text { Column } \\
\text { Foed } \\
\text { T. Sulfur } \\
\%\end{array}$ & \begin{tabular}{|c|} 
Measured \\
Column \\
Product \\
T. Sulfur \\
$\%$ \\
\end{tabular} & $\begin{array}{l}\text { Adjusted } \\
\text { Column } \\
\text { Product } \\
\text { T. Sulfur } \\
\%\end{array}$ & $\begin{array}{c}\text { Measured } \\
\text { Column } \\
\text { Reject } \\
\text { T. Sulfur } \\
\%\end{array}$ & $\begin{array}{c}\text { Adjusted } \\
\text { Column } \\
\text { Reject } \\
\text { T. Sulfur } \\
\%\end{array}$ \\
\hline & & & & & & \\
\hline 101 & 3.14 & 3.04 & 2.88 & 2.95 & 3.64 & 3.66 \\
\hline 102 & 2.31 & 2.51 & 2.78 & 2.54 & 2.44 & 2.40 \\
\hline 103 & 3.56 & 3.26 & 3.14 & 3.33 & 2.95 & 2.99 \\
\hline 104 & 2.96 & 3.05 & 3.11 & 3.03 & 3.21 & 3.20 \\
\hline 105 & 2.96 & 2.96 & 2.67 & 2.67 & 4.49 & 4.49 \\
\hline 106 & 3.37 & 2.93 & 2.61 & 2.79 & 3.09 & 3.21 \\
\hline 107 & 2.92 & 2.79 & 2.65 & 2.74 & 2.99 & 3.01 \\
\hline 108 & 2.79 & 2.82 & 2.80 & 2.78 & 3.04 & 3.04 \\
\hline 109 & 2.78 & 2.78 & 2.77 & 2.77 & 2.81 & 2.81 \\
\hline 110 & 2.85 & 2.78 & 2.83 & 2.89 & 2.18 & 2.19 \\
\hline 111 & 2.68 & 2.90 & 2.74 & 2.54 & 5.10 & 4.98 \\
\hline 113 & 2.68 & 2.76 & 2.74 & 2.66 & 5.10 & 5.09 \\
\hline 114 & 3.29 & 3.45 & 3.42 & 3.28 & 4.21 & 4.16 \\
\hline 116 & 2.83 & 3.00 & 3.16 & 2.97 & 3.24 & 3.21 \\
\hline 117 & 2.30 & 2.22 & 2.19 & 2.26 & 1.94 & 1.95 \\
\hline
\end{tabular}

Microcel - Pyritic Sulfur

\begin{tabular}{|c|c|c|c|c|c|c|}
\hline $\begin{array}{c}\text { Test } \\
\text { Number }\end{array}$ & $\begin{array}{c}\text { Measured } \\
\text { Column } \\
\text { Feed } \\
\text { Pyritic } s \\
\% \\
\end{array}$ & $\begin{array}{l}\text { Adjusted } \\
\text { Column } \\
\text { Feed } \\
\text { Pyritic } \mathbf{S} \\
\% \\
\end{array}$ & $\begin{array}{c}\text { Measured } \\
\text { Column } \\
\text { Product } \\
\text { Pyrtic S } \\
\% \\
\end{array}$ & $\begin{array}{c}\text { Adjustod } \\
\text { Column } \\
\text { Product } \\
\text { Pyritic } 8 \\
\% \\
\end{array}$ & $\begin{array}{c}\text { Measured } \\
\text { Column } \\
\text { Reject } \\
\text { Pyritlc } \mathbf{3} \\
\% \\
\end{array}$ & $\begin{array}{c}\text { Adjusted } \\
\text { Column } \\
\text { Roject } \\
\text { Pyritic S } \\
\% \\
\end{array}$ \\
\hline 101 & 155 & 156 & 138 & 1.37 & 298 & 2.97 \\
\hline 102 & 0.91 & 0.95 & 0.81 & 0.78 & 1.66 & 1.63 \\
\hline 103 & 1.64 & 1.56 & 1.32 & 1.36 & 2.39 & 2.42 \\
\hline 104 & 1.50 & 1.66 & 1.62 & 1.46 & 2.99 & 2.90 \\
\hline 105 & 1.53 & 1.55 & 1.20 & 1.19 & 3.45 & 3.43 \\
\hline 106 & 0.96 & 0.91 & 0.40 & 0.41 & 1.90 & 1.96 \\
\hline 107 & 1.40 & 1.34 & 1.15 & 1.18 & 2.22 & 2.24 \\
\hline 108 & 1.43 & 1.44 & 1.31 & 1.30 & 2.22 & 2.22 \\
\hline 109 & 0.83 & 0.88 & 0.69 & 0.66 & 2.09 & 2.05 \\
\hline 110 & 1.00 & 1.05 & 0.99 & 0.95 & 1.58 & 1.56 \\
\hline 111 & 1.13 & 1.04 & 0.43 & 0.44 & 4.23 & 4.43 \\
\hline 113 & 0.75 & 0.86 & 1.05 & 0.84 & 1.52 & 1.50 \\
\hline 114 & 1.66 & 1.07 & 0.47 & 0.51 & 3.11 & 3.50 \\
\hline 116 & 0.93 & 0.87 & 0.61 & 0.63 & 2.62 & 2.67 \\
\hline 117 & 0.59 & 0.41 & 0.26 & 0.29 & 1.18 & 1.27 \\
\hline
\end{tabular}




\section{Microcel/MGS Independent Tests Results - Pittsburgh \#8}

Microcel Performance

\begin{tabular}{||r||r|r|r||r|r||r|r||}
\hline $\begin{array}{c}\text { Test } \\
\text { Number }\end{array}$ & \multicolumn{1}{|c|}{$\begin{array}{c}\text { Comb. } \\
\text { Recovery }\end{array}$} & $\begin{array}{c}\text { Ash } \\
\text { Rejection }\end{array}$ & $\begin{array}{c}\text { Ash } \\
\text { Separation } \\
\text { Efficiency }\end{array}$ & $\begin{array}{r}\text { T. Sulfur } \\
\text { Rejection }\end{array}$ & $\begin{array}{c}\text { T. Sulfur } \\
\text { Separation } \\
\text { Efficiency }\end{array}$ & $\begin{array}{c}\text { Pyritic S } \\
\text { Rejection }\end{array}$ & $\begin{array}{c}\text { Pyritic S } \\
\text { Separation } \\
\text { Efficiency }\end{array}$ \\
\hline 101 & 96.72 & 45.32 & 42.04 & 14.58 & 11.29 & 22.69 & 19.41 \\
\hline 102 & 91.19 & 66.97 & 58.16 & 18.65 & 9.84 & 33.99 & 25.19 \\
\hline 103 & 93.98 & 65.68 & 59.66 & 16.95 & 10.93 & 29.12 & 23.10 \\
\hline 104 & 96.47 & 51.96 & 48.44 & 14.48 & 10.95 & 24.29 & 20.76 \\
\hline 105 & 95.73 & 62.19 & 57.92 & 24.25 & 19.99 & 35.53 & 31.26 \\
\hline 106 & 77.92 & 77.16 & 55.08 & 35.97 & 13.90 & 69.71 & 47.63 \\
\hline 107 & 95.42 & 59.74 & 55.16 & 16.89 & 12.31 & 25.48 & 20.90 \\
\hline 108 & 95.95 & 59.74 & 55.70 & 16.19 & 12.14 & 23.25 & 19.20 \\
\hline 109 & 94.79 & 56.63 & 51.43 & 15.70 & 10.50 & 36.55 & 31.34 \\
\hline 110 & 95.23 & 56.11 & 51.34 & 11.99 & 7.23 & 23.41 & 18.64 \\
\hline 111 & 95.40 & 54.51 & 49.91 & 25.47 & 20.87 & 64.00 & 59.40 \\
\hline 113 & 98.73 & 16.64 & 15.38 & 7.95 & 6.69 & 6.72 & 5.45 \\
\hline 114 & 92.53 & 62.49 & 55.02 & 22.86 & 15.39 & 61.32 & 53.86 \\
\hline 116 & 96.67 & 47.61 & 44.28 & 12.60 & 9.27 & 36.07 & 32.74 \\
\hline 117 & 94.78 & 56.87 & 51.65 & 11.00 & 5.77 & 38.16 & 32.94 \\
\hline
\end{tabular}




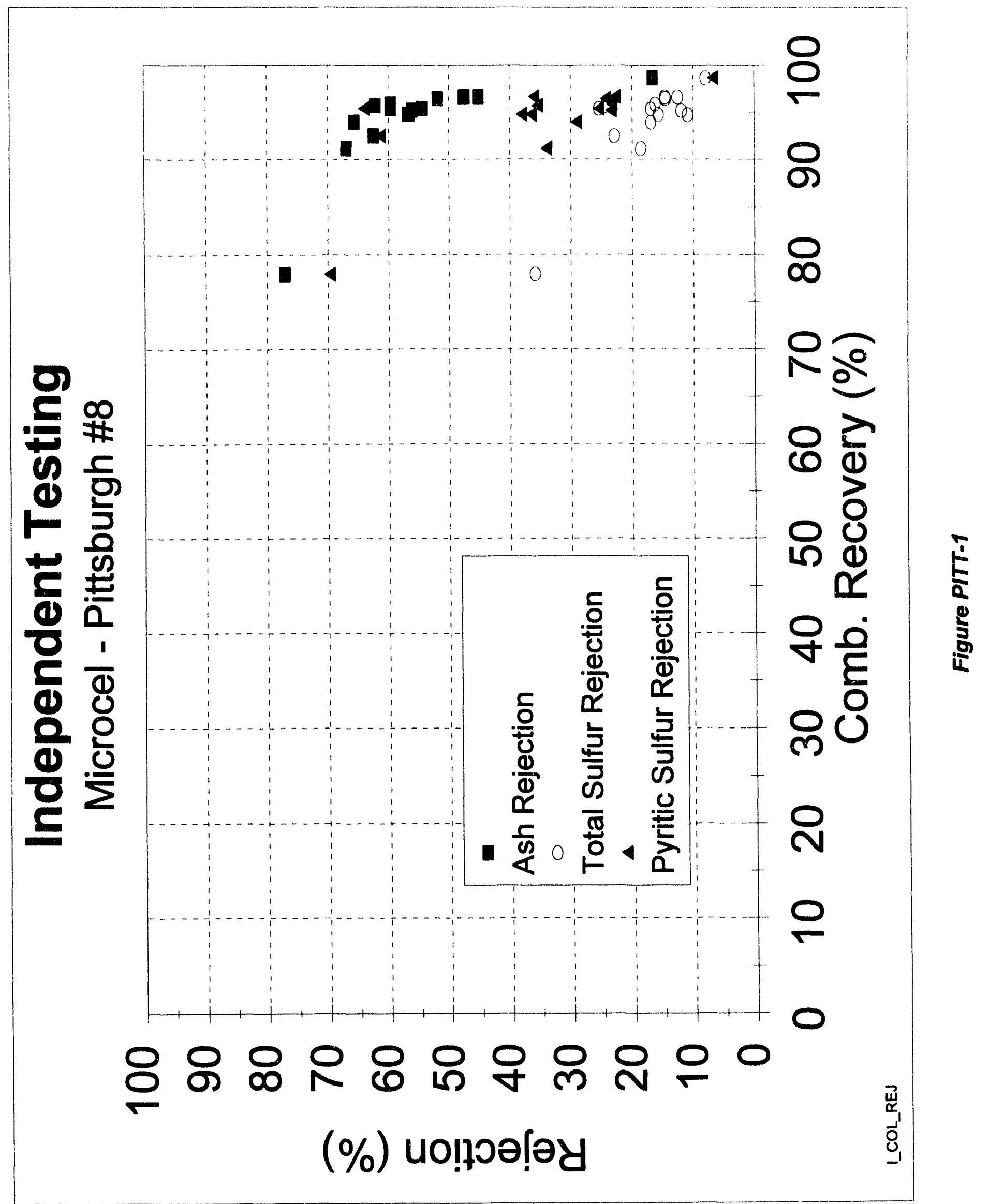


Microcel/MGS Independent Testing - Pittsburgh \#8

MGS Conditions Held Constant

Shake Amplitude (mm):

Shake Frequency (cps):

15

Tilt Angle (degrees):

MGS Variables

\begin{tabular}{|c|c|c|c|c|c|}
\hline $\begin{array}{c}\text { Test } \\
\text { Number }\end{array}$ & $\begin{array}{c}\text { Grind } \\
\text { Size } \\
\text { (microns) }\end{array}$ & $\begin{array}{c}\text { Drum } \\
\text { Speed } \\
\text { rpm }\end{array}$ & $\begin{array}{l}\text { Wash } \\
\text { Water } \\
\text { Ipm }\end{array}$ & $\begin{array}{l}\text { Percent } \\
\text { Feed } \\
\text { Solids } \\
\text { w/w }\end{array}$ & $\begin{array}{l}\text { Feed } \\
\text { Rate } \\
\text { lbs/hr }\end{array}$ \\
\hline & & & & & \\
\hline 201 & 200 & 280 & 1.02 & 35 & 110 \\
\hline 202 & 200 & 280 & 1.02 & 35 & 235 \\
\hline 203 & 200 & 241 & 1.48 & 26 & 169 \\
\hline 204 & 200 & 280 & 0.53 & 25 & 273 \\
\hline 205 & 200 & 240 & 0.53 & 25 & 159 \\
\hline 206 & 200 & 280 & 0.53 & 25 & 100 \\
\hline 207 & 200 & 280 & 0.95 & 26 & 200 \\
\hline 208 & 200 & 321 & 1.10 & 15 & 173 \\
\hline 209 & 200 & 280 & 0.91 & 16 & 261 \\
\hline 210 & 300 & 281 & 0.95 & 35 & 216 \\
\hline 211 & 300 & 280 & 0.98 & 25 & 260 \\
\hline 212 & 300 & 280 & 1.51 & 26 & 215 \\
\hline 213 & 300 & 280 & 0.91 & 25 & 110 \\
\hline 214 & 300 & 321 & 0.95 & 25 & 217 \\
\hline 215 & 300 & 281 & 0.45 & 26 & 215 \\
\hline 216 & 300 & 241 & 1.02 & 25 & 217 \\
\hline 217 & 300 & 280 & 1.02 & 14 & 239 \\
\hline 218 & 200 & 282 & 0.49 & 35 & 210 \\
\hline 219 & 200 & 280 & 1.51 & 35 & 216 \\
\hline 220 & 200 & 320 & 0.98 & 25 & 334 \\
\hline 221 & 200 & 240 & 0.98 & 24 & 347 \\
\hline 222 & 200 & 280 & 0.98 & 26 & 164 \\
\hline 223 & 200 & 240 & 0.98 & 26 & 137 \\
\hline 224 & 200 & 281 & 1.51 & 25 & 313 \\
\hline 225 & 200 & 281 & 1.48 & 16 & 200 \\
\hline 226 & 200 & 242 & 0.98 & 15 & 212 \\
\hline 227 & 100 & 284 & 0.98 & 36 & 197 \\
\hline 228 & 100 & 282 & 0.91 & 25 & 100 \\
\hline 229 & 100 & 280 & 0.98 & 26 & 321 \\
\hline 230 & 100 & 320 & 0.98 & 26 & 198 \\
\hline 231 & 100 & 280 & 0.49 & 25 & 204 \\
\hline 232 & 100 & 240 & 0.98 & 25 & 208 \\
\hline 233 & 100 & 280 & 1.48 & 26 & 200 \\
\hline 234 & 100 & 280 & 0.98 & 15 & 205 \\
\hline 235 & 200 & 320 & 0.95 & 35 & 208 \\
\hline 236 & 200 & 241 & 0.95 & 35 & 223 \\
\hline 237 & 200 & 320 & 0.98 & 25 & 128 \\
\hline 238 & 200 & 280 & 0.98 & 26 & 176 \\
\hline 239 & 200 & 321 & 0.53 & 26 & 186 \\
\hline 240 & 200 & 321 & 1.51 & 25 & 146 \\
\hline 241 & 200 & 280 & 1.59 & 25 & 103 \\
\hline 242 & 200 & 280 & 0.42 & 15 & 236 \\
\hline 243 & 200 & 280 & 0.91 & 16 & 119 \\
\hline
\end{tabular}




\title{
Microcel/MGS Independent Tests Results - Pittsburgh \#8
}

\author{
MGS - Ash
}

\begin{tabular}{|c|c|c|c|c|c|c|c|c|}
\hline $\begin{array}{c}\text { Test } \\
\text { Number } \\
\end{array}$ & $\begin{array}{c}\text { Measured } \\
\text { MGS } \\
\text { Feed } \\
\text { Ash } \\
\% \\
\end{array}$ & $\begin{array}{c}\text { Adjusted } \\
\text { MGS } \\
\text { Feed } \\
\text { Ash } \\
\% \\
\end{array}$ & $\begin{array}{c}\text { Measured } \\
\text { MGS } \\
\text { Lights } \\
\text { Ash } \\
\% \\
\end{array}$ & $\begin{array}{c}\text { Adjusted } \\
\text { MGS } \\
\text { Lights } \\
\text { Ash } \\
\% \\
\end{array}$ & $\begin{array}{c}\text { Measured } \\
\text { MGS } \\
\text { Heavies } \\
\text { Ash } \\
\% \\
\end{array}$ & $\begin{array}{c}\text { Adjusted } \\
\text { MGS } \\
\text { Heavies } \\
\text { Ash } \\
\% \\
\end{array}$ & $\begin{array}{c}\text { Measured } \\
\text { MGS } \\
\text { Ash } \\
\text { Yield } \\
\% \\
\end{array}$ & $\begin{array}{c}\text { Adjusted } \\
\text { MGS } \\
\text { Ash } \\
\text { Yiold } \\
\%\end{array}$ \\
\hline & & & & & & & & \\
\hline 201 & 18.40 & 18.42 & 12.52 & 12.52 & 63.86 & 63.84 & \begin{tabular}{|c|}
88.55 \\
\end{tabular} & 88.50 \\
\hline 202 & 19.36 & 19.34 & 12.57 & 12.58 & 57.80 & 57.90 & 84.99 & 85.08 \\
\hline 203 & 17.49 & $17.5 \mathrm{e}$ & 16.13 & 16.06 & 73.80 & 73.76 & 97.64 & 97.37 \\
\hline 204 & 20.84 & 20.80 & 19.31 & 14.33 & 54.04 & 54.08 & 95.59 & 83.72 \\
\hline 205 & 21.41 & 21.45 & 19.63 & 19.60 & 76.10 & 76.08 & 96.85 & 96.72 \\
\hline 206 & 23.02 & 22.97 & 15.00 & 15.02 & 73.45 & 73.52 & 86.28 & 86.41 \\
\hline 207 & 21.29 & 21.24 & 15.76 & 15.79 & 74.51 & 74.57 & 90.59 & 90.73 \\
\hline 208 & 18.14 & 18.18 & 13.92 & 13.90 & 65.58 & 65.53 & 91.83 & 91.71 \\
\hline 209 & 17.71 & 17.77 & 14.13 & 14.10 & 44.80 & 44.75 & 88.33 & 88.03 \\
\hline 210 & 19.65 & 19.66 & 12.23 & 12.23 & 49.77 & 49.68 & 80.23 & 80.16 \\
\hline 211 & 19.92 & 19.96 & 19.15 & 19.11 & 73.80 & 73.79 & 98.59 & 98.45 \\
\hline 212 & 20.80 & 20.77 & 13.70 & 13.71 & 59.04 & 59.08 & 84.34 & 84.44 \\
\hline 213 & 21.23 & 21.20 & 13.79 & 13.80 & 61.81 & 61.85 & 84.51 & 84.60 \\
\hline 214 & 19.80 & 19.79 & 12.43 & 12.43 & 41.03 & 41.04 & 74.23 & 74.27 \\
\hline 215 & 20.94 & 20.92 & 13.93 & 13.94 & 51.80 & 51.82 & 81.49 & 81.57 \\
\hline 216 & 20.54 & 20.50 & 18.58 & 18.61 & 74.54 & 74.56 & 96.50 & 96.62 \\
\hline 217 & 20.06 & 20.06 & 17.44 & 17.44 & 70.40 & 70.40 & 95.05 & 95.05 \\
\hline 248 & 20.52 & 20.52 & 12.57 & 12.57 & 48.26 & 48.26 & 77.72 & 77.72 \\
\hline 219 & 20.23 & 20.21 & 13.00 & 13.00 & 56.13 & 56.15 & 83.24 & 83.29 \\
\hline 220 & 20.31 & 20.28 & 12.61 & 12.62 & 46.60 & 46.64 & 77.35 & 77.48 \\
\hline 221 & 20.68 & 20.67 & 17.63 & 17.64 & 69.14 & 69.15 & 94.08 & 94.12 \\
\hline 222 & 21.51 & 21.48 & 14.22 & 14.23 & 56.77 & 56.81 & 82.87 & 82.97 \\
\hline 223 & 24.72 & 24.12 & 22.97 & 23.48 & 70.14 & 70.21 & 96.29 & 98.63 \\
\hline 224 & 19.73 & 19.73 & 14.05 & 14.05 & 56.52 & 56.52 & 86.63 & 86.63 \\
\hline 225 & 19.14 & 19.13 & 16.07 & 16.09 & 70.74 & 70.75 & 94.38 & 94.44 \\
\hline 226 & 20.06 & 20.06 & 19.30 & 19.30 & 72.52 & 72.52 & 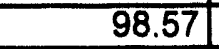 & 98.57 \\
\hline 227 & 19.69 & 19.70 & 13.50 & 13.50 & 55.22 & 55.21 & 85.16 & 85.14 \\
\hline 228 & 20.70 & 20.69 & 14.73 & 14.74 & 69.12 & 69.14 & 89.02 & 89.06 \\
\hline 229 & 20.89 & 20.88 & 16.71 & 16.72 & 57.92 & 57.93 & 89.86 & 89.91 \\
\hline 230 & 20.87 & 20.86 & 14.54 & 14.54 & 51.24 & 51.25 & 82.75 & 82.78 \\
\hline 231 & 20.24 & 20.28 & 15.40 & 15.42 & 57.05 & 57.01 & 88.38 & 88.31 \\
\hline 232 & 20.19 & 20.18 & 17.42 & 17.43 & 75.38 & 75.39 & 95.22 & 95.26 \\
\hline 233 & 19.62 & 19.61 & 14.64 & 14.64 & 64.25 & 64.26 & 89.96 & 89.98 \\
\hline 234 & 19.75 & 19.76 & 16.43 & 16.42 & 66.11 & 66.10 & 93.32 & 93.28 \\
\hline 235 & 29.22 & 29.15 & 11.31 & 11.31 & 39.65 & 39.73 & 36.80 & 37.23 \\
\hline 236 & 20.15 & 20.18 & 15.99 & 15.97 & 72.73 & 72.70 & 92.67 & 92.58 \\
\hline 237 & 22.30 & 22.25 & 14.08 & 14.10 & 65.96 & 66.03 & 84.16 & 84.31 \\
\hline 238 & 21.00 & 20.98 & 15.07 & 15.07 & 65.01 & 65.03 & 88.13 & 88.17 \\
\hline 239 & 21.01 & 21.01 & 12.46 & 12.46 & 47.61 & 47.61 & 75.68 & 75.68 \\
\hline 240 & 21.00 & 21.00 & 14.09 & 14.09 & 63.50 & 63.49 & 86.01 & 86.01 \\
\hline 241 & 24.47 & 24.52 & 17.77 & 17.75 & 70.92 & 70.87 & 87.39 & 87.26 \\
\hline 242 & 20.99 & 21.07 & 17.30 & 17.25 & 65.34 & 65.28 & 92.32 & 92.05 \\
\hline 243 & 19.75 & 19.79 & 13.47 & 13.45 & 65.16 & 65.11 & 87.85 & 87.73 \\
\hline
\end{tabular}




\section{Microcel/MGS Independent Tests Results - Pittsburgh \#8}

MGS - Total Sulfur

\begin{tabular}{|c|c|c|c|c|c|c|}
\hline $\begin{array}{c}\text { Test } \\
\text { Number }\end{array}$ & $\begin{array}{c}\text { Measured } \\
\text { MGS } \\
\text { Feed } \\
\text { T. Sulfur } \\
\% \\
\end{array}$ & $\begin{array}{c}\text { Adjusted } \\
\text { MGS } \\
\text { Foed } \\
\text { T. Sulfur } \\
\% \\
\end{array}$ & $\begin{array}{c}\text { Measured } \\
\text { MGS } \\
\text { Lights } \\
\text { T. Sulfur } \\
\% \\
\end{array}$ & $\begin{array}{c}\text { Adjusted } \\
\text { MGS } \\
\text { Lights } \\
\text { T. Sulfur } \\
\% \\
\end{array}$ & $\begin{array}{c}\text { Measured } \\
\text { MGS } \\
\text { Heavies } \\
\text { T. Sulfur } \\
\% \\
\end{array}$ & $\begin{array}{c}\text { Adjusted } \\
\text { MGS } \\
\text { Heavies } \\
\text { T. Sulfur } \\
\% \\
\end{array}$ \\
\hline & & & & & & \\
\hline 201 & 2.72 & 2.60 & 1.76 & 1.81 & 8.56 & 8.70 \\
\hline 202 & 2.83 & 2.90 & 1.75 & 1.73 & 9.71 & 9.59 \\
\hline 203 & 2.73 & 2.73 & 2.03 & 2.03 & 28.57 & 28.67 \\
\hline 204 & 2.74 & 2.86 & 1.80 & 1.76 & 8.71 & 8.52 \\
\hline 205 & 2.89 & 2.83 & 2.04 & 2.07 & 25.40 & 25.54 \\
\hline 206 & 2.98 & 3.18 & 1.81 & 1.75 & 12.73 & 12.25 \\
\hline 207 & 2.86 & 2.92 & 1.90 & 1.87 & 13.30 & 13.17 \\
\hline 208 & 2.82 & 2.66 & 1.85 & 1.91 & 10.89 & 10.99 \\
\hline 209 & 2.48 & 2.48 & 1.88 & 1.88 & 6.95 & 6.95 \\
\hline 210 & 2.96 & 2.88 & 1.79 & 1.81 & 7.06 & 7.16 \\
\hline 211 & 2.89 & 2.79 & 2.41 & 2.48 & 22.15 & 22.24 \\
\hline 212 & 2.96 & 3.00 & 1.93 & 1.92 & 8.91 & 8.86 \\
\hline 213 & 3.11 & 3.08 & 2.04 & 2.05 & 8.72 & 8.75 \\
\hline 214 & 3.09 & 3.00 & 1.83 & 1.85 & 6.21 & 6.31 \\
\hline 215 & 3.04 & 3.00 & 1.97 & 1.98 & 7.48 & 7.52 \\
\hline 216 & 3.05 & 3.09 & 2.37 & 2.35 & 24.42 & 24.34 \\
\hline 217 & 3.56 & 3.03 & 2.10 & 2.28 & 16.91 & 17.50 \\
\hline 218 & 3.04 & 2.96 & 1.82 & 1.84 & 6.76 & 6.85 \\
\hline 219 & 2.89 & 2.98 & 1.89 & 1.86 & 8.69 & 8.56 \\
\hline 220 & 2.76 & 2.91 & 1.88 & 1.83 & 6.87 & 6.66 \\
\hline 221 & 2.79 & 2.80 & 2.05 & 2.05 & 14.76 & 14.75 \\
\hline 222 & 2.69 & 2.77 & 1.81 & 1.78 & 7.66 & 7.56 \\
\hline 223 & 3.38 & 3.01 & 2.42 & 2.61 & 31.61 & 32.05 \\
\hline 224 & 2.98 & 2.91 & 1.85 & 1.87 & 9.57 & 9.66 \\
\hline 225 & 2.65 & 2.65 & 1.96 & 1.96 & 14.32 & 14.32 \\
\hline 226 & 2.87 & 2.87 & 2.43 & 2.43 & 32.87 & 32.88 \\
\hline 227 & 2.76 & 2.67 & 1.68 & 1.71 & 8.08 & 8.19 \\
\hline 228 & 2.85 & 2.77 & 1.76 & 1.79 & 10.66 & 10.78 \\
\hline 229 & 2.77 & 2.76 & 1.87 & 1.87 & 10.66 & 10.72 \\
\hline 230 & 2.78 & 2.77 & 1.76 & 1.76 & 7.62 & 7.63 \\
\hline 231 & 2.92 & 2.70 & 1.79 & 1.86 & 8.78 & 9.01 \\
\hline 232 & 2.90 & 2.86 & 1.95 & 1.97 & 20.80 & 20.89 \\
\hline 233 & 2.76 & 2.69 & 1.78 & 1.81 & 10.50 & 10.60 \\
\hline 234 & 2.82 & 2.77 & 1.93 & 1.95 & 14.08 & 14.16 \\
\hline 235 & 4.46 & 4.25 & 1.68 & 1.69 & 5.55 & 5.76 \\
\hline 236 & 2.95 & 2.92 & 1.95 & 1.96 & 14.85 & 14.90 \\
\hline 237 & 2.99 & 3.06 & 1.85 & 1.83 & 9.79 & 9.67 \\
\hline 238 & 2.96 & 3.00 & 1.91 & 1.89 & 11.39 & 11.31 \\
\hline 239 & 3.05 & 3.00 & 1.79 & 1.80 & 6.65 & 6.71 \\
\hline 240 & 3.05 & 3.03 & 1.87 & 1.88 & 10.07 & 10.10 \\
\hline 241 & 3.55 & 3.43 & 2.00 & 2.03 & 12.79 & 12.99 \\
\hline 242 & 2.99 & 2.89 & 1.98 & 2.02 & 12.81 & 12.96 \\
\hline 243 & 2.77 & 2.76 & 1.84 & 1.85 & 9.25 & 9.27 \\
\hline
\end{tabular}




\section{Microcel/MGS Independent Tests Results - Pittsburgh \#8}

MGS - Pyritic Sulfur

\begin{tabular}{|c|c|c|c|c|c|c|}
\hline $\begin{array}{c}\text { Test } \\
\text { Number }\end{array}$ & $\begin{array}{c}\text { Moasured } \\
\text { MGS } \\
\text { Feed } \\
\text { Pyritic S } \\
\%\end{array}$ & $\begin{array}{c}\text { Adjusted } \\
\text { MGS } \\
\text { Feed } \\
\text { Pyritic S } \\
\%\end{array}$ & $\begin{array}{c}\text { Measured } \\
\text { MGS } \\
\text { Lights } \\
\text { Pyritic S } \\
\%\end{array}$ & $\begin{array}{l}\text { Adjusted } \\
\text { MGS } \\
\text { Lights } \\
\text { Pyritic S } \\
\%\end{array}$ & $\begin{array}{c}\text { Measured } \\
\text { MGS } \\
\text { Heavies } \\
\text { Pyritic S } \\
\%\end{array}$ & $\begin{array}{c}\text { Adjusted } \\
\text { MGS } \\
\text { Heavles } \\
\text { Pyritic S } \\
\%\end{array}$ \\
\hline & & & & & & \\
\hline 201 & \begin{tabular}{|l|}
1.26 \\
\end{tabular} & 1.29 & 0.45 & 0.45 & 7.84 & 7.73 \\
\hline 202 & 1.35 & 1.41 & 0.43 & 0.42 & 7.29 & 7.03 \\
\hline 203 & 1.24 & 1.39 & 0.73 & 0.68 & 30.11 & 27.76 \\
\hline 204 & 1.42 & 1.51 & 0.50 & 0.49 & 7.17 & 6.78 \\
\hline 205 & 1.58 & 1.53 & 0.73 & 0.74 & 24.26 & 24.68 \\
\hline 206 & 1.30 & 1.71 & 0.54 & 0.50 & 12.24 & 9.35 \\
\hline 207 & 1.38 & 1.55 & 0.56 & 0.54 & 12.74 & 11.43 \\
\hline 208 & 1.45 & 1.35 & 0.50 & 0.51 & 10.15 & 10.57 \\
\hline 209 & 1.17 & 1.21 & 0.59 & 0.58 & 5.93 & 5.81 \\
\hline 210 & 1.53 & 1.51 & 0.47 & 0.47 & 5.63 & 5.69 \\
\hline 211 & 1.18 & 1.14 & 0.81 & 0.83 & 21.00 & 21.18 \\
\hline 212 & 1.26 & 1.40 & 0.46 & 0.44 & 7.33 & 6.59 \\
\hline 213 & 1.17 & 1.34 & 0.47 & 0.45 & 7.22 & 6.23 \\
\hline 214 & 1.18 & 1.30 & 0.36 & 0.35 & 4.51 & 4.05 \\
\hline 215 & 1.42 & 1.53 & 0.51 & 0.50 & 6.53 & 6.10 \\
\hline 216 & 1.34 & 1.43 & 0.75 & 0.72 & 22.40 & 21.59 \\
\hline 217 & 1.34 & 1.42 & 0.76 & 0.74 & 14.92 & 14.45 \\
\hline 218 & 1.44 & 1.51 & 0.46 & 0.45 & 5.38 & 5.17 \\
\hline 219 & 1.41 & 1.40 & 0.48 & 0.48 & 6.36 & 6.25 \\
\hline 220 & 1.36 & 1.43 & 0.48 & 0.47 & 4.93 & 4.72 \\
\hline 221 & 1.47 & 1.51 & 0.76 & 0.75 & 13.93 & 13.71 \\
\hline 222 & 1.44 & 1.55 & 0.57 & 0.56 & 6.85 & 6.41 \\
\hline 223 & 1.89 & 1.69 & 1.19 & 1.27 & 32.56 & 31.75 \\
\hline 224 & 1.29 & 1.36 & 0.43 & 0.42 & 7.72 & 7.40 \\
\hline 225 & 1.08 & 1.14 & 0.52 & 0.51 & 12.36 & 11.91 \\
\hline 226 & 1.14 & 1.14 & 0.77 & 0.77 & 27.05 & 27.02 \\
\hline 227 & 1.27 & 1.31 & 0.40 & 0.40 & 6.72 & 6.55 \\
\hline 228 & 1.07 & 1.20 & 0.36 & 0.35 & 9.20 & 8.15 \\
\hline 229 & 1.19 & 1.26 & 0.48 & 0.47 & 8.67 & 8.30 \\
\hline 230 & 1.44 & 1.49 & 0.48 & 0.48 & 6.57 & 6.38 \\
\hline 231 & 1.24 & 1.20 & 0.42 & 0.42 & 6.94 & 7.08 \\
\hline 232 & 1.28 & 1.34 & 0.56 & 0.55 & 17.88 & 17.28 \\
\hline 233 & 1.28 & 1.37 & 0.51 & 0.50 & 9.68 & 9.18 \\
\hline 234 & 0.98 & 1.13 & 0.48 & 0.45 & 12.02 & 10.54 \\
\hline 235 & 3.62 & 2.96 & 0.37 & 0.37 & 3.99 & 4.49 \\
\hline 236 & 1.32 & 1.48 & 0.64 & 0.60 & 13.76 & 12.45 \\
\hline 237 & 1.06 & 1.23 & 0.31 & 0.30 & 7.69 & 6.26 \\
\hline 238 & 1.62 & 1.68 & 0.61 & 0.60 & 9.94 & 9.69 \\
\hline 239 & 1.27 & 1.31 & 0.30 & 0.30 & 4.61 & 4.47 \\
\hline 240 & 1.76 & 1.76 & 0.57 & 0.57 & 9.02 & 9.04 \\
\hline 241 & 2.24 & 2.12 & 0.70 & 0.71 & 12.18 & 11.79 \\
\hline 242 & 1.53 & 1.61 & 0.70 & 0.68 & 11.40 & 12.35 \\
\hline 243 & 1.28 & 1.38 & 0.56 & 0.54 & 7.80 & 7.35 \\
\hline
\end{tabular}




\section{Microcel/MGS Independent Tests Results - Pittsburgh \#8}

MGS - Performance

\begin{tabular}{|c|c|c|c|c|c|c|c|}
\hline $\begin{array}{c}\text { Test } \\
\text { Number }\end{array}$ & $\begin{array}{l}\text { Comb. } \\
\text { Recovery }\end{array}$ & \begin{tabular}{c|} 
Ash \\
Rejection \\
$\%$ \\
\end{tabular} & $\begin{array}{c}\text { Ash } \\
\text { Soparation } \\
\text { Efficlency } \\
\% \\
\end{array}$ & $\begin{array}{l}\text { T.Sulfur } \\
\text { Rejection }\end{array}$ & $\begin{array}{c}\text { T.Sulfur } \\
\text { Soparation } \\
\text { Efficioncy } \\
\% \\
\end{array}$ & $\begin{array}{l}\text { Pyritic S } \\
\text { Rejection }\end{array}$ & $\begin{array}{c}\text { Pyritic S } \\
\text { Separation } \\
\text { Efficioncy } \\
\% \\
\end{array}$ \\
\hline & & & & & & & \\
\hline 201 & 94.90 & 39.84 & 34.75 & 38.39 & 33.29 & 69.13 & 64.03 \\
\hline 202 & 92.21 & 44.66 & 36.87 & 49.24 & 41.46 & 74.66 & 66.87 \\
\hline 203 & 99.16 & 11.05 & 10.21 & 27.60 & 26.76 & 52.37 & 51.53 \\
\hline 204 & 90.56 & 42.32 & 32.88 & 48.48 & 39.04 & 72.83 & 63.39 \\
\hline 205 & 99.00 & 11.62 & 10.62 & 29.25 & 28.25 & 53.22 & 52.22 \\
\hline 206 & 95.33 & 43.50 & 38.83 & 52.45 & 47.78 & 74.73 & 70.06 \\
\hline 207 & 97.01 & 32.55 & 29.56 & 41.90 & 38.90 & 68.39 & 65.40 \\
\hline 208 & 96.51 & 29.88 & 26.39 & 34.15 & 30.66 & 65.35 & 61.86 \\
\hline 209 & 91.95 & 30.15 & 22.11 & 33.27 & 25.23 & 57.81 & 49.76 \\
\hline 210 & 87.57 & 50.13 & 37.71 & 49.62 & 37.20 & 75.05 & 62.62 \\
\hline 211 & 99.49 & 5.75 & 5.24 & 12.49 & 11.98 & 28.32 & 27.82 \\
\hline 212 & 91.96 & 44.26 & 36.23 & 45.96 & 37.92 & 73.46 & 65.43 \\
\hline 213 & 92.54 & 44.93 & 37.47 & 43.69 & 36.24 & 71.59 & 64.13 \\
\hline 214 & 81.09 & 53.35 & 34.44 & 54.20 & 35.29 & 80.00 & 61.09 \\
\hline 215 & 88.77 & 45.64 & 34.42 & 46.16 & 34.94 & 73.34 & 62.12 \\
\hline 216 & 98.92 & 12.29 & 11.21 & 26.52 & 25.44 & 51.35 & 50.27 \\
\hline 217 & 98.17 & 17.36 & 15.53 & 28.48 & 26.64 & 50.47 & 48.63 \\
\hline 218 & 85.50 & 52.39 & 37.89 & 51.68 & 37.18 & 76.84 & 62.34 \\
\hline 219 & 90.82 & 46.42 & 37.24 & 48.01 & 38.83 & 71.44 & 62.26 \\
\hline 220 & 84.93 & 51.78 & 36.71 & 51.27 & 36.20 & 74.53 & 59.46 \\
\hline 221 & 97.71 & 19.68 & 17.39 & 31.09 & 28.80 & 53.25 & 50.97 \\
\hline 222 & 90.63 & 45.03 & 35.67 & 46.68 & 37.32 & 70.02 & 60.66 \\
\hline 223 & 99.46 & 3.99 & 3.45 & 14.48 & 13.94 & 25.88 & 25.34 \\
\hline 224 & 92.76 & 38.31 & 31.07 & 44.33 & 37.09 & 73.25 & 66.00 \\
\hline 225 & 97.99 & 20.57 & 18.56 & 30.15 & 28.14 & 57.75 & 55.74 \\
\hline 226 & 99.51 & $\frac{5.16}{5.16}$ & 4.67 & 16.54 & 16.05 & 33.42 & 32.93 \\
\hline 227 & 91.71 & 41.66 & 33.37 & 45.48 & 37.18 & 74.00 & 65.71 \\
\hline 228 & 95.74 & 36.55 & 32.29 & 42.45 & 38.19 & 74.02 & 69.77 \\
\hline 229 & 94.63 & 28.01 & 22.64 & 39.09 & 33.72 & 66.46 & 61.10 \\
\hline 230 & 89.39 & 42.30 & 31.69 & 47.40 & 36.80 & 73.33 & 62.73 \\
\hline 231 & 93.70 & 32.85 & 26.55 & 39.16 & 32.86 & 69.09 & 62.79 \\
\hline 232 & 98.54 & 17.73 & 16.26 & 34.39 & 32.92 & 60.90 & 59.44 \\
\hline 233 & 95.55 & 32.82 & 28.37 & 39.45 & 35.00 & 67.16 & 62.71 \\
\hline 234 & 97.16 & 22.49 & 19.65 & 34.34 & 31.50 & 62.85 & 60.01 \\
\hline 235 & 46.60 & 85.56 & 32.16 & 85.20 & 31.80 & 95.35 & 41.95 \\
\hline 236 & 97.46 & 26.74 & 24.20 & 37.86 & 35.32 & 62.47 & 59.93 \\
\hline 237 & 93.14 & 46.57 & 39.72 & 49.58 & 42.72 & 79.44 & 72.58 \\
\hline 238 & 94.76 & 36.67 & 31.43 & 44.45 & 39.22 & 68.51 & 63.28 \\
\hline 239 & 83.87 & 55.12 & 38.99 & 54.59 & 38.46 & 82.67 & 66.54 \\
\hline 240 & 93.54 & 42.29 & 35.83 & 46.63 & 40.17 & 72.14 & 65.68 \\
\hline 241 & 95.08 & 36.84 & 31.92 & 48.36 & 43.44 & 70.78 & 65.86 \\
\hline 242 & 96.50 & 24.64 & 21.14 & 35.66 & 32.16 & 61.12 & 57.62 \\
\hline 243 & 94.66 & 40.38 & 35.04 & 41.20 & 35.86 & 65.67 & 60.33 \\
\hline
\end{tabular}




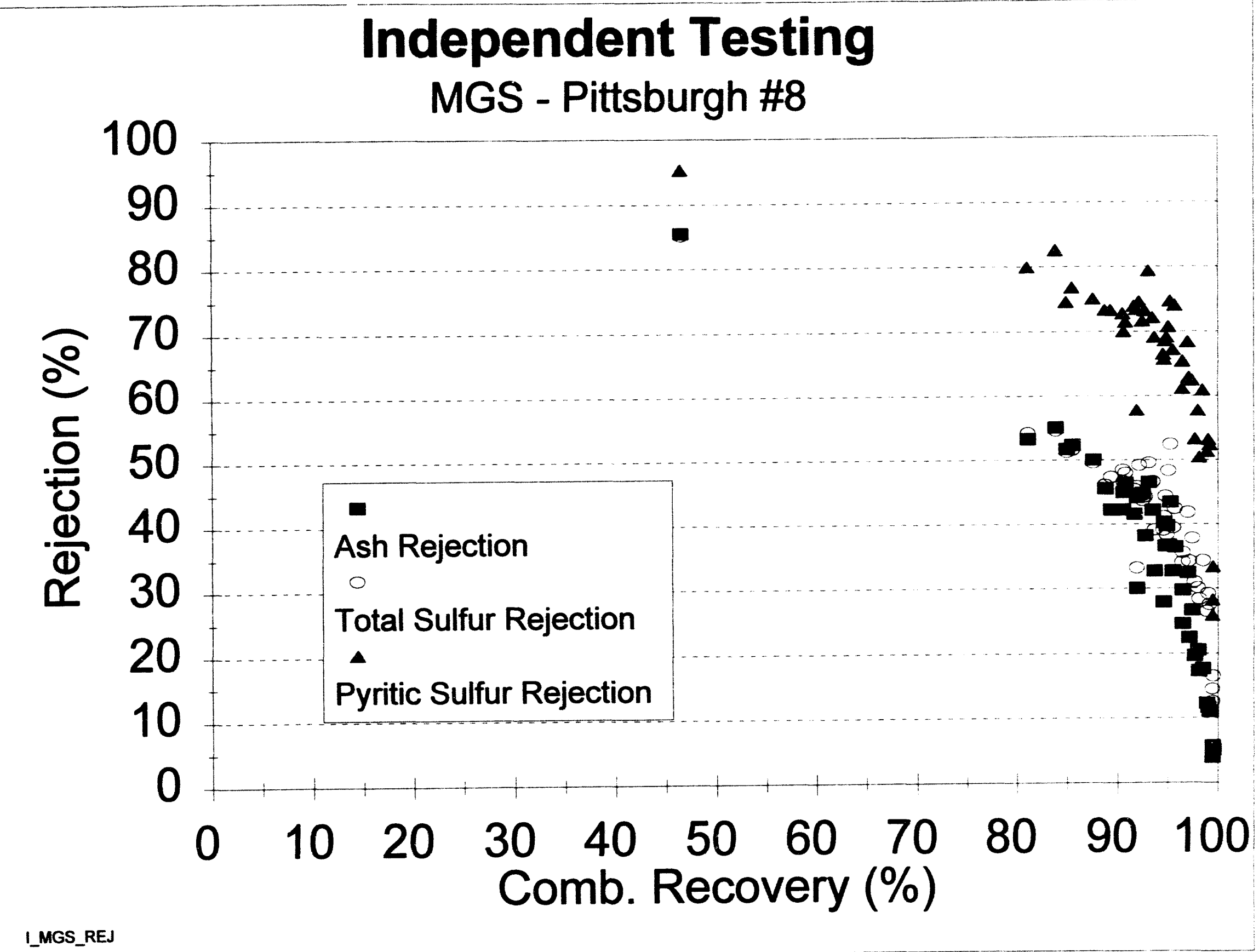

Figure PITT-2 


\section{Microcel/MgS Combined Testing - Pittsburgh \#8}

\begin{tabular}{lc}
\multicolumn{2}{c}{ Microcel } \\
Wash Water (Ipm): & 40.12 \\
Percent Solids (w/w): & $14.5-15.5$ \\
Grind Size (d80 (microns)): & 200 \\
Air Flow Rate (SCFM): & 8.89 \\
Diesel dosage (Ibs/ton): & 1 \\
Frother Dosage (ml/min): & 1
\end{tabular}

MGS

Percent Solids (w/w):

Shake Frequency (cps):

$18-22$

Shake Amplitude (mm):

Tilt Angle (degrees):

MGS Variables

\begin{tabular}{|c|c|c|c|c|}
\hline $\begin{array}{c}\text { Test } \\
\text { Number }\end{array}$ & $\begin{array}{l}\text { Column } \\
\text { Feod } \\
\text { (lbs/hr) }\end{array}$ & $\begin{array}{l}\text { Drum } \\
\text { Speed } \\
\text { rpm }\end{array}$ & $\begin{array}{l}\text { Wash } \\
\text { Water } \\
\text { Ipm }\end{array}$ & $\begin{array}{l}\text { MGS } \\
\text { Feed } \\
\text { lbs/hr }\end{array}$ \\
\hline & & & & \\
\hline 301 & 512 & 282 & 0.5 & 249 \\
\hline 302 & 527 & 283 & 1.02 & 378 \\
\hline 303 & 415 & 241 & 1.51 & 259 \\
\hline 304 & 392 & 242 & 0.98 & 373 \\
\hline 305 & 414 & 280 & 0.49 & 381 \\
\hline 306 & 427 & 282 & 1.06 & 260 \\
\hline 307 & 420 & 322 & 1.50 & 241 \\
\hline 308 & 414 & 242 & 0.98 & 124 \\
\hline 309 & 415 & 320 & 0.98 & 351 \\
\hline 310 & 391 & 280 & 0.49 & 135 \\
\hline 311 & 397 & 280 & 1.51 & 393 \\
\hline 312 & 410 & 280 & 1.55 & 134 \\
\hline 313 & 458 & 280 & 0.95 & 259 \\
\hline 314 & 302 & 280 & 0.98 & 160 \\
\hline 315 & 333 & 241 & 1.00 & 267 \\
\hline 316 & 529 & 281 & 0.98 & 141 \\
\hline 317 & 474 & 241 & 0.98 & 276 \\
\hline 318 & 491 & 320 & 0.98 & 267 \\
\hline 319 & 491 & 282 & 1.55 & 285 \\
\hline 320 & 444 & 242 & 0.49 & 326 \\
\hline 321 & 383 & 280 & 0.95 & 313 \\
\hline 322 & 427 & 320 & 0.51 & 297 \\
\hline 323 & 393 & 320 & 0.97 & 170 \\
\hline 324 & 311 & 281 & 0.51 & 268 \\
\hline 325 & 31 & 281 & 1.55 & 285 \\
\hline 326 & 29 & 280 & 0.95 & 474 \\
\hline 327 & 324 & 320 & 0.98 & $\overline{328}$ \\
\hline
\end{tabular}


Combined Microcel/MGS Tests Results - Pittsburgh \#8

Ash

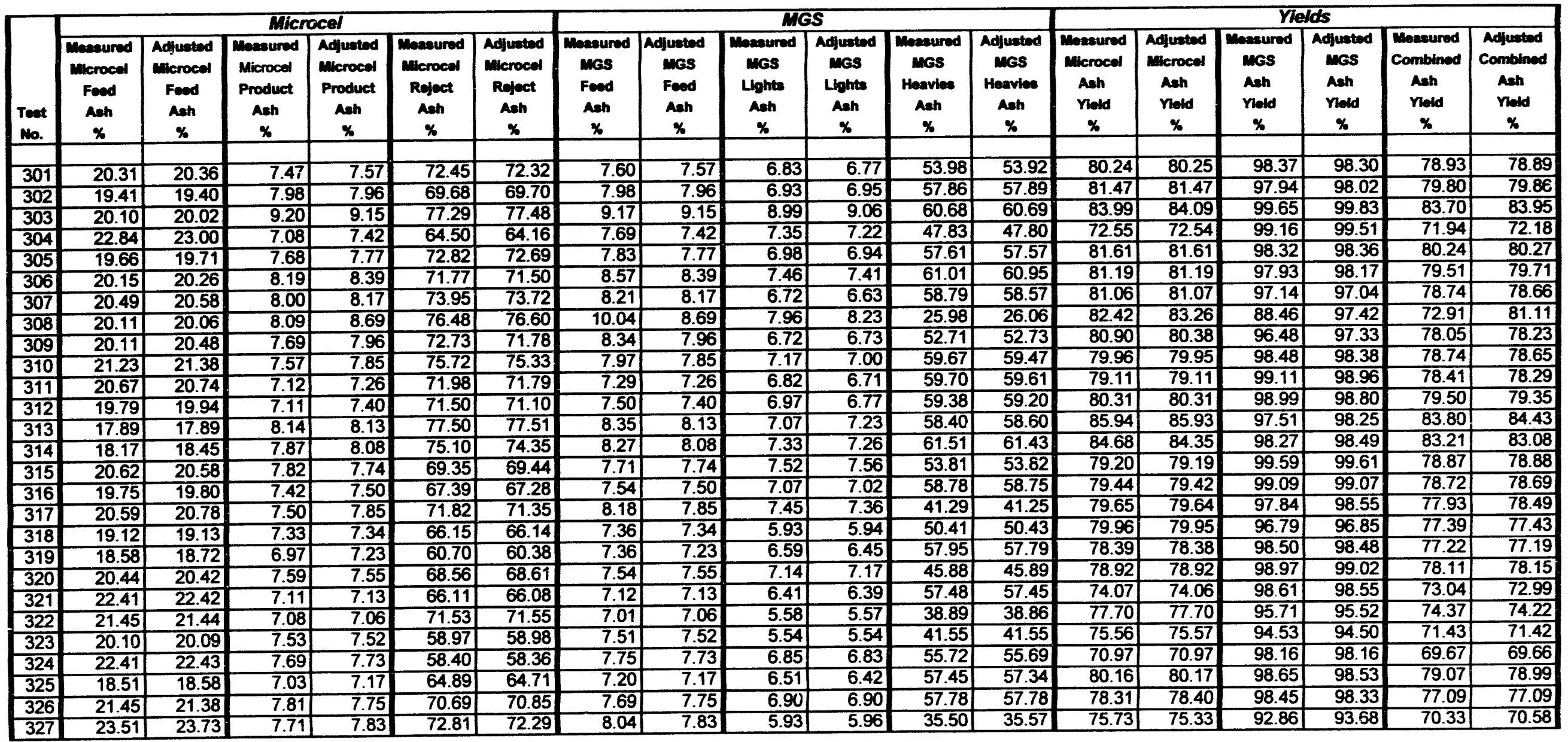

Table PITT-10 


\section{Combined Microcel/MGS Tests Results - Pittsburgh \#8}

\section{Total Sulfur}

\begin{tabular}{|c|c|c|c|c|c|c|c|c|c|c|c|c|}
\hline \multirow[b]{2}{*}{$\begin{array}{c}\text { Test } \\
\text { Number }\end{array}$} & \multicolumn{6}{|c|}{ Microcel } & \multicolumn{6}{|c|}{ MGS } \\
\hline & $\begin{array}{c}\text { Measured } \\
\text { Microcel } \\
\text { Feed } \\
\text { T. Sulfur } \\
\% \\
\end{array}$ & $\begin{array}{c}\text { Adjusted } \\
\text { Microcel } \\
\text { Feed } \\
\text { T. Sulfur } \\
\% \\
\end{array}$ & \begin{tabular}{|c|} 
Measured \\
Microcel \\
Product \\
T. Sulfur \\
$\%$ \\
\end{tabular} & $\begin{array}{c}\text { Adjusted } \\
\text { Microcel } \\
\text { Product } \\
\text { T. Sulfur } \\
\% \\
\end{array}$ & $\begin{array}{c}\text { Measured } \\
\text { Microcel } \\
\text { Reject } \\
\text { T. Sulfur } \\
\% \\
\end{array}$ & $\begin{array}{c}\text { Adjusted } \\
\text { Microcel } \\
\text { Reject } \\
\text { T. Sulfur } \\
\% \\
\end{array}$ & $\begin{array}{c}\text { Measured } \\
\text { MGS } \\
\text { Feed } \\
\text { T. Sulfur } \\
\% \\
\end{array}$ & $\begin{array}{c}\text { Adjusted } \\
\text { MGS } \\
\text { Feed } \\
\text { T. Sulfur } \\
\% \\
\end{array}$ & $\begin{array}{c}\text { Measured } \\
\text { MGS } \\
\text { Lights } \\
\text { T. Sulfur } \\
\% \\
\end{array}$ & $\begin{array}{l}\text { Adjusted } \\
\text { MGS } \\
\text { Lights } \\
\text { T. Sulfur } \\
\% \\
\end{array}$ & $\begin{array}{c}\text { Measured } \\
\text { MGS } \\
\text { Heavies } \\
\text { T. Sulfur } \\
\% \\
\end{array}$ & $\begin{array}{c}\text { Adjusted } \\
\text { MGs } \\
\text { Heavies } \\
\text { T. Sulfur } \\
\% \\
\end{array}$ \\
\hline & & & & & & & & & & & & \\
\hline 301 & 2.58 & 2.46 & 2.36 & 2.42 & 2.60 & 2.62 & 2.43 & 2.42 & 1.85 & 1.87 & 34.60 & 34.71 \\
\hline 302 & 2.60 & 2.57 & 2.46 & 2.47 & 3.03 & 3.04 & 2.43 & 2.47 & 1.82 & 1.80 & 35.50 & 35.37 \\
\hline 303 & 2.52 & 2.54 & 2.51 & 2.54 & 2.54 & 2.54 & 2.45 & 2.54 & 2.62 & 2.47 & 43.00 & 42.92 \\
\hline 304 & 2.50 & 2.57 & 2.32 & 2.47 & 2.86 & 2.84 & 2.49 & 2.47 & 2.51 & 2.31 & 35.90 & 35.70 \\
\hline 305 & 2.50 & 2.45 & 2.36 & 2.40 & 2.68 & 2.69 & 2.38 & 2.40 & 1.84 & 1.83 & 36.50 & 36.43 \\
\hline 306 & 2.62 & 2.53 & 2.61 & 2.48 & 2.71 & 2.73 & 2.47 & 2.48 & 1.79 & 1.87 & 34.70 & 35.28 \\
\hline 307 & 2.67 & 2.57 & 2.66 & 2.54 & 2.68 & 2.70 & 2.55 & 2.54 & 1.70 & 1.78 & 26.70 & 27.33 \\
\hline 308 & 2.67 & 2.79 & 2.58 & 2.62 & 3.62 & 3.58 & 2.56 & 2.62 & 2.37 & 2.21 & 19.00 & 18.73 \\
\hline 309 & 2.54 & 2.59 & 2.53 & 2.56 & 2.74 & 2.73 & 2.71 & 2.56 & 1.88 & 1.92 & 25.70 & 25.88 \\
\hline 310 & 2.70 & 2.66 & 2.56 & 2.60 & 2.90 & 2.91 & 2.65 & 2.60 & 1.92 & 1.94 & 42.13 & 42.29 \\
\hline 311 & 2.90 & 2.65 & 2.54 & 2.60 & 2.78 & 2.83 & 2.63 & 2.60 & 2.08 & 2.15 & 44.60 & 44.96 \\
\hline 312 & 2.71 & 2.72 & 2.52 & 2.50 & 3.59 & 3.59 & 2.57 & 2.50 & 1.92 & 1.96 & 46.70 & 47.01 \\
\hline 313 & 2.67 & 2.71 & 2.62 & 2.68 & 2.91 & 2.90 & 2.63 & 2.68 & 2.07 & 1.98 & 42.50 & 41.85 \\
\hline 314 & 2.67 & 2.52 & 2.55 & 2.45 & 2.91 & 2.94 & 2.55 & 2.45 & 1.72 & 1.86 & 38.80 & 39.94 \\
\hline 315 & 2.59 & 2.59 & 2.44 & 2.44 & 3.15 & 3.15 & 2.44 & 2.44 & 2.29 & 2.29 & 41.70 & 41.69 \\
\hline 316 & 2.33 & 2.34 & 2.14 & 2.19 & 2.38 & 2.40 & 2.20 & 2.19 & 1.85 & 1.86 & 37.40 & 37.44 \\
\hline 317 & 2.31 & 2.40 & 2.09 & 2.23 & 3.07 & 3.04 & 2.18 & 2.23 & 2.08 & 1.84 & 29.90 & 29.18 \\
\hline 318 & 2.45 & 2.23 & 2.08 & 2.16 & 2.46 & 2.50 & 2.10 & 2.16 & 1.52 & 1.52 & 22.00 & 21.97 \\
\hline 319 & 2.52 & 2.50 & 2.43 & 2.46 & 2.63 & 2.64 & 2.47 & 2.46 & 1.87 & 1.87 & 41.00 & 40.99 \\
\hline 320 & 2.70 & 2.61 & 2.49 & 2.54 & 2.82 & 2.84 & 2.52 & 2.54 & 2.25 & 2.24 & 33.04 & 33.02 \\
\hline 321 & 3.03 & 2.91 & 2.45 & 2.44 & 4.18 & 4.24 & 2.44 & 2.44 & 1.88 & 1.92 & 37.13 & 37.38 \\
\hline 322 & 3.06 & 2.74 & 2.41 & 2.44 & 3.70 & 3.80 & 2.39 & 2.44 & 1.71 & 1.75 & 17.12 & 17.28 \\
\hline 323 & 2.40 & 2.48 & 2.39 & 2.41 & 2.70 & 2.68 & 2.46 & 2.41 & 1.69 & 1.67 & 15.30 & 15.21 \\
\hline 324 & 2.83 & 2.65 & 2.44 & 2.47 & 3.02 & 3.08 & 2.45 & 2.47 & 1.89 & 1.92 & 31.10 & 31.26 \\
\hline 325 & 2.51 & 2.50 & 2.40 & 2.45 & 2.72 & 2.72 & 2.52 & 2.45 & 1.88 & 1.89 & 39.92 & 39.99 \\
\hline 326 & 2.76 & 2.64 & 2.54 & 2.50 & 3.11 & 3.14 & 2.44 & 2.50 & 1.89 & 1.92 & 36.65 & 36.81 \\
\hline 327 & 2.71 & 2.70 & 2.48 & 253 & 3.23 & 3.23 & 2.55 & 2.53 & \begin{tabular}{l|l|}
1.71 \\
\end{tabular} & 1.70 & 14.93 & 14.87 \\
\hline
\end{tabular}

Table PITT-11 


\section{Combined Microcel/MGS Tests Results - Pittsburgh \#8}

Pyritic Sulfur

\begin{tabular}{|c|c|c|c|c|c|c|c|c|c|c|c|c|}
\hline \multirow[b]{2}{*}{$\begin{array}{c}\text { Test } \\
\text { Number }\end{array}$} & \multicolumn{6}{|c|}{ Microcel } & \multicolumn{6}{|c|}{ MGS } \\
\hline & $\begin{array}{l}\text { Measured } \\
\text { Microcel } \\
\text { Foed } \\
\text { Pyritic S } \\
\% \\
\end{array}$ & $\begin{array}{c}\text { Adjusted } \\
\text { Microcel } \\
\text { Feed } \\
\text { Pyritic S } \\
\% \\
\end{array}$ & \begin{tabular}{|c|}
$\begin{array}{c}\text { Measured } \\
\text { Microcel } \\
\text { Product } \\
\text { Pyritic S } \\
\%\end{array}$ \\
\end{tabular} & $\begin{array}{c}\text { Adjusted } \\
\text { Microcel } \\
\text { Product } \\
\text { Pyritic S } \\
\% \\
\end{array}$ & $\begin{array}{c}\text { Measured } \\
\text { Microcel } \\
\text { Reject } \\
\text { Pyritic S } \\
\% \\
\end{array}$ & $\begin{array}{c}\text { Adjusted } \\
\text { Microcel } \\
\text { Reject } \\
\text { Pyritic S } \\
\% \\
\end{array}$ & $\begin{array}{c}\text { Measured } \\
\text { MGS } \\
\text { Feed } \\
\text { Pyritic S } \\
\% \\
\end{array}$ & $\begin{array}{c}\text { Adjusted } \\
\text { MGS } \\
\text { Feed } \\
\text { Pyritic S } \\
\% \\
\end{array}$ & \begin{tabular}{|c|} 
Measured \\
MGS \\
Lights \\
Pyritic S \\
$\%$
\end{tabular} & $\begin{array}{c}\text { Adjusted } \\
\text { MGS } \\
\text { Lights } \\
\text { Pyritic S } \\
\% \\
\end{array}$ & \begin{tabular}{|c|} 
Measured \\
MGS \\
Heavies \\
Pyritic S \\
$\%$ \\
\end{tabular} & $\begin{array}{c}\text { Adjusted } \\
\text { MGS } \\
\text { Heavies } \\
\text { Pyritic S } \\
\% \\
\end{array}$ \\
\hline & & & & & & & & & & & & \\
\hline 301 & 1.30 & 1.28 & 1.03 & 1.10 & 1.95 & 1.99 & 1.15 & 1.10 & 0.57 & 0.58 & 31.40 & 31.74 \\
\hline 302 & 1.34 & 1.35 & 1.07 & 1.16 & 2.18 & 2.18 & 1.13 & 1.16 & 0.58 & 0.54 & 34.18 & 31.65 \\
\hline 303 & 1.34 & 1.14 & 1.03 & 1.06 & 1.50 & 1.54 & 0.99 & 1.06 & 1.01 & 0.99 & 39.77 & 39.73 \\
\hline 304 & 1.19 & 1.21 & 0.89 & 0.93 & 1.96 & 1.94 & 1.03 & 0.93 & 0.84 & 0.86 & 17.09 & 17.12 \\
\hline 305 & 1.33 & 1.14 & 0.90 & 0.92 & 2.02 & 2.10 & 0.99 & 0.92 & 0.58 & 0.63 & 17.73 & 18.45 \\
\hline 306 & 1.30 & 1.34 & 1.27 & 1.19 & 2.03 & 2.01 & 1.12 & 1.19 & 0.58 & 0.57 & 34.89 & 34.34 \\
\hline 307 & 1.40 & 1.26 & 1.09 & 1.13 & 1.78 & 1.82 & 1.01 & 1.13 & 0.45 & 0.43 & 26.01 & 24.07 \\
\hline 308 & 1.21 & 1.42 & 1.06 & 1.16 & 2.93 & 2.72 & 1.13 & 1.16 & 1.07 & 0.81 & 15.89 & 14.43 \\
\hline 309 & 1.26 & 1.27 & 1.06 & 1.10 & 1.97 & 1.97 & 1.06 & 1.10 & 0.50 & 0.48 & 24.59 & 23.46 \\
\hline 310 & 1.37 & 1.34 & 1.00 & 1.15 & 2.10 & 2.11 & 1.13 & 1.15 & 0.61 & 0.55 & 41.05 & 36.91 \\
\hline 311 & 1.26 & 1.18 & 0.92 & 0.97 & 1.95 & 1.99 & 1.06 & 0.97 & 0.55 & 0.57 & 37.94 & 38.86 \\
\hline 312 & 1.07 & 1.23 & 0.83 & 0.94 & 2.62 & 2.43 & 0.96 & 0.94 & 0.59 & 0.51 & 41.28 & 36.36 \\
\hline 313 & 0.93 & 1.04 & 0.95 & 0.98 & 1.43 & 1.39 & 0.88 & 0.98 & 0.55 & 0.47 & 35.37 & 29.55 \\
\hline 314 & 0.96 & 1.14 & 0.94 & 1.03 & 1.82 & 1.72 & 1.13 & 1.03 & 0.57 & 0.51 & 38.45 & 34.35 \\
\hline 315 & 1.16 & 1.35 & 1.39 & 1.10 & 2.49 & 2.31 & 1.02 & 1.10 & 1.00 & 0.96 & 38.00 & 37.75 \\
\hline 316 & 1.15 & 1.15 & 0.88 & 0.99 & 1.77 & 1.77 & 1.02 & 0.99 & 0.69 & 0.64 & 40.28 & 38.56 \\
\hline 317 & 1.19 & 1.25 & 0.90 & 1.00 & 2.28 & 2.23 & 0.92 & 1.00 & 0.81 & 0.64 & 29.29 & 26.06 \\
\hline 318 & 1.15 & 1.10 & 0.90 & 1.00 & 1.50 & 1.52 & 0.90 & 1.00 & 0.49 & 0.44 & 21.40 & 18.25 \\
\hline 319 & 1.07 & 1.22 & 0.93 & 1.09 & 1.78 & 1.69 & 2.47 & 1.09 & 0.57 & 0.55 & 37.31 & 36.08 \\
\hline 320 & 1.49 & 1.40 & 1.17 & 1.20 & 2.12 & 2.16 & 1.11 & 1.20 & 0.97 & 0.91 & 32.28 & 31.57 \\
\hline 321 & 1.90 & 1.79 & 1.13 & 1.13 & 3.55 & 3.65 & 1.11 & 9.13 & 0.60 & 0.60 & 37.11 & 37.17 \\
\hline 322 & 1.91 & 1.64 & 1.05 & 1.12 & 3.28 & 3.46 & 1.05 & 1.12 & 0.42 & 0.41 & 17.08 & 16.25 \\
\hline 323 & 1.19 & 1.32 & 1.06 & 1.10 & 2.10 & 2.00 & 1.12 & 1.10 & 0.36 & 0.35 & 14.87 & 13.91 \\
\hline 324 & 1.40 & 1.37 & 1.07 & 1.07 & 2.07 & 2.09 & 1.04 & 1.07 & 0.53 & 0.53 & 29.94 & 29.70 \\
\hline 325 & 1.10 & 1.12 & 0.95 & 0.99 & 1.66 & 1.65 & 1.00 & 0.99 & 0.51 & 0.50 & 34.39 & 33.66 \\
\hline 326 & 1.39 & 1.37 & 1.03 & 1.08 & 2.39 & 2.40 & 1.06 & 1.08 & 0.57 & 0.55 & 33.06 & 31.99 \\
\hline 327 & 1.36 & 1.40 & 0.90 & 1.08 & 2.44 & 2.40 & 1.22 & 1.08 & 0.42 & 0.40 & 12.58 & 11.17 \\
\hline
\end{tabular}

Table PITT-12 
Combined Microcel/MGS Tests Results - Pittsburgh \#8

Ash Performance

\begin{tabular}{|c|c|c|c|c|c|c|c|c|c|}
\hline \multirow[b]{2}{*}{$\begin{array}{c}\text { Test } \\
\text { Number }\end{array}$} & \multicolumn{3}{|c|}{ Microcel } & \multicolumn{3}{|c|}{ MGS } & \multicolumn{3}{|c|}{ Combined Microcel/MGS } \\
\hline & $\begin{array}{c}\text { Comb. } \\
\text { Recovery } \\
\% \\
\end{array}$ & $\begin{array}{c}\begin{array}{c}\text { Ash } \\
\text { Rejection }\end{array} \\
\% \\
\end{array}$ & $\begin{array}{c}\text { Ash } \\
\text { Separation } \\
\text { Efficiency } \\
\% \\
\end{array}$ & $\begin{array}{c}\text { Comb. } \\
\text { Recovery } \\
\% \\
\end{array}$ & $\begin{array}{c}\text { Ash } \\
\text { Rejection } \\
\% \\
\end{array}$ & \begin{tabular}{c|} 
Ash \\
Separation \\
Efficiency \\
$\%$ \\
\end{tabular} & $\begin{array}{c}\text { Comb. } \\
\text { Recovery } \\
\% \\
\end{array}$ & $\begin{array}{c}\text { Ash } \\
\text { Rejection } \\
\% \\
\end{array}$ & \begin{tabular}{|c|} 
Ash \\
Separation \\
Efficiency \\
$\%$ \\
\end{tabular} \\
\hline & & & & & & & & & \\
\hline 301. & 93.13 & 70.16 & 63.30 & 99.15 & 12.09 & 11.24 & 92.35 & 73.77 & 66.12 \\
\hline 302. & 93.03 & 66.57 & 59.61 & 99.09 & 14.42 & 13.51 & 92.19 & 71.39 & 63.58 \\
\hline 303 & 95.52 & 61.57 & 57.09 & 99.92 & 1.16 & 1.08 & 95.45 & 62.01 & 57.46 \\
\hline 304 & 87.22 & 76.60 & 63.82 & 99.72 & 3.17 & 2.90 & 86.98 & 77.34 & 64.32 \\
\hline 305 & 93.74 & 67.83 & 61.57 & 99.25 & 12.15 & 11.39 & 93.04 & 71.74 & 64.77 \\
\hline 306 & 93.28 & 66.38 & 59.65 & 99.22 & 13.30 & 12.52 & 92.55 & 70.85 & 63.40 \\
\hline 307 & 93.74 & 67.82 & 61.55 & 98.66 & 21.26 & 19.92 & 92.48 & 74.66 & 67.14 \\
\hline 308 & 95.10 & 63.93 & 59.03 & 97.91 & 7.74 & 5.65 & 93.11 & 66.72 & 59.84 \\
\hline 309 & 93.04 & 68.76 & 61.80 & 98.63 & 17.71 & 16.34 & 91.76 & 74.29 & 66.05 \\
\hline 310 & 93.71 & 70.65 & 64.35 & 99.29 & 12.27 & 11.56 & 93.04 & 74.25 & 67.29 \\
\hline 311 & 92.57 & 72.31 & 64.87 & 99.55 & 8.54 & 8.08 & 92.15 & 74.67 & 66.82 \\
\hline 312 & 92.89 & 70.19 & 63.09 & 99.47 & 9.61 & 9.08 & 92.40 & 73.06 & 65.46 \\
\hline 313 & 96.15 & 60.95 & 57.10 & 99.21 & 12.63 & 11.84 & 95.39 & 65.88 & 61.27 \\
\hline 314 & 95.08 & 63.06 & 58.14 & 99.36 & 11.51 & 10.87 & 94.47 & 67.31 & 61.78 \\
\hline 315 & 91.99 & 70.22 & 62.21 & 99.81 & 2.71 & 2.51 & 91.81 & 71.02 & 62.84 \\
\hline 316 & \begin{tabular}{l|l}
91.61 \\
\end{tabular} & 69.91 & 61.52 & 99.59 & 7.27 & 6.85 & 91.23 & 72.10 & 63.33 \\
\hline 317 & 92.64 & 69.92 & 62.55 & 99.08 & 7.60 & 6.68 & 91.78 & 72.20 & 63.98 \\
\hline 318 & 91.60 & 69.32 & 60.93 & 98.32 & 21.62 & 19.94 & 90.06 & 75.96 & 66.02 \\
\hline 319 & 89.46 & 69.73 & 59.19 & 99.31 & 12.14 & 11.45 & 88.84 & 73.40 & 62.25 \\
\hline 320 & 91.69 & 70.82 & 62.51 & 99.43 & 5.97 & 5.39 & 91.16 & 72.56 & 63.72 \\
\hline 321 & 88.66 & 76.45 & 65.11 & 99.34 & 11.68 & 11.01 & 88.07 & 79.20 & 67.27 \\
\hline 322 & 91.92 & 74.41 & 66.34 & 97.06 & 24.64 & 21.69 & 89.22 & 80.72 & 69.94 \\
\hline 323 & 87.46 & 71.71 & 59.17 & 96.52 & 30.38 & 26.91 & 84.42 & 80.31 & 64.73 \\
\hline 324 & 84.41 & 75.54 & 59.96 & 99.12 & 13.27 & 12.39 & 83.67 & 78.79 & 62.46 \\
\hline 325 & 91.41 & 69.06 & 60.47 & 99.32 & 11.78 & 11.10 & 90.79 & 72.71 & 63.49 \\
\hline 326 & 91.99 & 71.58 & 63.57 & 99.24 & 12.46 & 11.69 & 91.29 & 75.12 & 66.41 \\
\hline 327 & 91.04 & 75.14 & 66.18 & 95.59 & 28.69 & 24.27 & 87.02 & 82.27 & 69.29 \\
\hline
\end{tabular}

Table PITT-13 


\section{Combined Microcel/MGS Tests Results - Pittsburgh \#8}

Total Sulfur Performance

\begin{tabular}{|c|c|c|c|c|c|c|c|c|c|}
\hline \multirow[b]{2}{*}{$\begin{array}{c}\text { Test } \\
\text { Number }\end{array}$} & \multicolumn{3}{|c|}{ Microcel } & \multicolumn{3}{|c|}{ MGS } & \multicolumn{3}{|c|}{ Combined Microcel/MGS } \\
\hline & 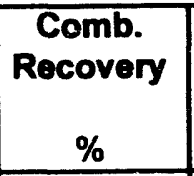 & $\begin{array}{c}\text { T. Sulfur } \\
\text { Rejection } \\
\% \\
\end{array}$ & \begin{tabular}{|c|} 
T. Sulfur \\
Separation \\
Efficiency \\
$\%$ \\
\end{tabular} & $\begin{array}{c}\text { Comb. } \\
\text { Recovery } \\
\% \\
\end{array}$ & $\begin{array}{c}\text { T. Sulfur } \\
\text { Rejection } \\
\% \\
\end{array}$ & \begin{tabular}{|c|} 
T. Sulfur \\
Separation \\
Efficiency \\
$\%$ \\
\end{tabular} & $\begin{array}{c}\text { Comb. } \\
\text { Recovery } \\
\% \\
\end{array}$ & $\begin{array}{c}\text { T. Sulfur } \\
\text { Rejection } \\
\% \\
\end{array}$ & \begin{tabular}{|c|} 
T. Sulfur \\
Separation \\
Efficiency \\
$\%$ \\
\end{tabular} \\
\hline & & & & & & & & & \\
\hline 301 & 93.13 & 21.06 & 14.19 & 99.15 & 24.04 & 23.19 & 92.35 & 40.03 & 32.38 \\
\hline 302 & 93.03 & 21.70 & 14.73 & 99.09 & 28.57 & 27.66 & 92.19 & 44.07 & 36.26 \\
\hline 303 & 95.52 & 15.91 & 11.43 & 99.92 & 2.93 & 2.85 & 95.45 & 18.37 & 13.82 \\
\hline 304 & 87.22 & 30.28 & 17.50 & 99.72 & 6.94 & 6.66 & 86.98 & 35.12 & 22.10 \\
\hline 305 & 93.74 & 20.06 & 13.80 & 99.25 & 25.00 & 24.25 & 93.04 & 40.04 & 33.08 \\
\hline 306 & 93.28 & 20.41 & 13.69 & 99.22 & 25.98 & 25.20 & 92.55 & 41.09 & 33.64 \\
\hline 307 & 93.74 & 19.88 & 13.61 & 98.66 & 32.00 & 30.66 & 92.48 & 45.52 & 38.00 \\
\hline 308 & 95.10 & 21.82 & 16.91 & 97.91 & 17.83 & 15.74 & 93.11 & 35.75 & 28.86 \\
\hline 309 & 93.04 & 20.55 & 13.59 & 98.63 & 27.01 & 25.63 & 91.76 & 42.00 & 33.77 \\
\hline 310 & 93.71 & 21.85 & 15.56 & 99.29 & 26.59 & 25.88 & 93.04 & 42.64 & 35.68 \\
\hline 311 & 92.57 & 22.38 & 14.95 & 99.55 & 18.17 & 17.71 & 92.15 & 36.48 & 28.63 \\
\hline 312 & 92.89 & 26.18 & 19.08 & 99.47 & 22.54 & 22.01 & 92.40 & 42.82 & 35.22 \\
\hline 313 & 96.15 & 15.02 & 11.17 & 99.21 & 27.41 & 26.62 & 95.39 & 38.32 & 33.70 \\
\hline 314 & 95.08 & 17.99 & 13.07 & 99.36 & 25.23 & 24.60 & 94.47 & 38.68 & 33.16 \\
\hline 315 & 91.99 & 25.40 & 17.39 & 99.81 & 6.51 & 6.32 & 91.81 & 30.26 & 22.07 \\
\hline 316 & 91.61 & 25.67 & 17.27 & 99.59 & 15.86 & 15.44 & 91.23 & 37.45 & 28.68 \\
\hline 317 & 92.64 & 26.00 & 18.64 & 99.08 & 18.68 & 17.76 & 91.78 & 39.83 & 31.61 \\
\hline 318 & 91.60 & 22.56 & 14.17 & 98.32 & 31.84 & 30.16 & 90.06 & 47.22 & 37.28 \\
\hline 319 & 89.46 & 22.87 & 12.33 & 99.31 & 25.14 & 24.45 & 88.84 & 42.26 & 31.10 \\
\hline 320 & 91.69 & 23.19 & 14.88 & 99.43 & 12.68 & 12.10 & 91.16 & 32.93 & 24.09 \\
\hline 321 & 88.66 & 37.90 & 26.56 & 99.34 & 22.45 & 21.79 & 88.07 & 51.84 & 39.91 \\
\hline 322 & 91.92 & 30.81 & 22.73 & 97.06 & 31.49 & 28.54 & 89.22 & 52.59 & 41.81 \\
\hline 323 & 87.46 & 26.56 & 14.02 & 96.52 & 34.52 & 31.04 & 84.42 & 51.91 & 36.33 \\
\hline 324 & 84.41 & 33.85 & 18.27 & 99.12 & 23.70 & 22.81 & 83.67 & 49.53 & 33.20 \\
\hline 325 & 91.41 & 21.43 & 12.84 & 99.32 & 23.99 & 23.32 & 90.79 & 40.28 & 31.07 \\
\hline 326 & 91.99 & 25.76 & 17.75 & 99.24 & 24.48 & 23.72 & 91.29 & 43.93 & 35.22 \\
\hline 327 & 91.04 & 29.41 & 20.45 & 95.59 & 37.05 & 32.64 & 87.02 & 55.56 & 42.58 \\
\hline
\end{tabular}

Table PITT-14 


\section{Combined Microcel/MGS Tests Results - Pittsburgh \#8}

Pyritic Sulfur Performance

\begin{tabular}{|c|c|c|c|c|c|c|c|c|c|}
\hline \multirow[b]{2}{*}{$\begin{array}{c}\text { Test } \\
\text { Number }\end{array}$} & \multicolumn{3}{|c|}{ Microcel } & \multicolumn{3}{|c|}{ MGS } & \multicolumn{3}{|c|}{ Combined Microcel/MGS } \\
\hline & $\begin{array}{c}\text { Comb. } \\
\text { Recovery } \\
\% \\
\end{array}$ & $\begin{array}{c}\text { Pyritic S } \\
\text { Rejection } \\
\%\end{array}$ & $\begin{array}{c}\text { Pyritic S } \\
\text { Separation } \\
\text { Efficiency } \\
\% \\
\end{array}$ & $\begin{array}{c}\text { Comb. } \\
\text { Recovery } \\
\% \\
\%\end{array}$ & $\begin{array}{c}\text { Pyritic S } \\
\text { Rejection } \\
\% \\
\end{array}$ & $\begin{array}{c}\text { Pyritic S } \\
\text { Separation } \\
\text { Efficiency } \\
\% \\
\end{array}$ & $\begin{array}{c}\text { Comb. } \\
\text { Recovery } \\
\% \\
\end{array}$ & $\begin{array}{c}\text { Pyritic } \mathbf{S} \\
\text { Rejection } \\
\% \\
\end{array}$ & $\begin{array}{c}\text { Pyritic S } \\
\text { Separation } \\
\text { Efficiency } \\
\% \\
\end{array}$ \\
\hline & & & & & & & & & \\
\hline 301 & 93.13 & 31.04 & 24.17 & 99.15 & 48.17 & 47.32 & 92.35 & 64.25 & 56.60 \\
\hline 302 & 93.03 & 30.00 & 23.03 & 99.09 & 54.37 & 53.46 & 92.19 & 68.06 & 60.25 \\
\hline 303 & 95.52 & 21.81 & 17.33 & 99.92 & 6.77 & 6.69 & 95.45 & 27.10 & 22.55 \\
\hline 304 & 87.22 & 44.25 & 31.46 & 99.72 & 7.98 & 7.70 & 86.98 & 48.70 & 35.67 \\
\hline 305 & 93.74 & 34.14 & 27.88 & 99.25 & 32.64 & 31.89 & 93.04 & 55.64 & 48.68 \\
\hline 306 & 93.28 & 27.90 & 21.17 & 99.22 & 52.98 & 52.20 & 92.55 & 66.10 & 58.65 \\
\hline 307 & 93.74 & 27.30 & 21.03 & 98.66 & 63.08 & 61.74 & 92.48 & 73.15 & 65.64 \\
\hline 308 & 95.10 & 31.99 & 27.09 & 97.91 & 31.97 & 29.88 & 93.11 & 53.73 & 46.85 \\
\hline 309 & 93.04 & 30.38 & 23.42 & 98.63 & 57.53 & 56.16 & 91.76 & 70.43 & 62.19 \\
\hline 310 & 93.71 & 31.39 & 25.09 & 99.29 & 52.95 & 52.24 & 93.04 & 67.72 & 60.76 \\
\hline 311 & 92.57 & 34.97 & 27.53 & 99.55 & 41.85 & 41.40 & 92.15 & 62.18 & 54.33 \\
\hline 312 & 92.89 & 38.62 & 31.52 & 99.47 & 46.40 & 45.87 & 92.40 & 67.10 & 59.50 \\
\hline 313 & 96.15 & 19.03 & 15.17 & 99.21 & 52.88 & 52.09 & 95.39 & 61.85 & 57.23 \\
\hline 314 & 95.08 & 23.79 & 18.87 & 99.36 & 51.23 & 50.60 & 94.47 & 62.83 & 57.31 \\
\hline 315 & 91.99 & 35.48 & 27.47 & 99.81 & 13.07 & 12.87 & 91.81 & 43.91 & 35.72 \\
\hline 316 & 91.61 & 31.63 & 23.23 & 99.59 & 35.95 & 35.54 & 91.23 & 56.21 & 47.44 \\
\hline 317 & 92.64 & 36.29 & 28.93 & 99.08 & 36.93 & 36.00 & 91.78 & 59.81 & 51.60 \\
\hline 318 & 91.60 & 27.32 & 18.92 & 98.32 & 57.38 & 55.70 & 90.06 & 69.03 & 59.09 \\
\hline 319 & 89.46 & 29.97 & 19.43 & 99.31 & 50.31 & 49.62 & 88.84 & 65.20 & 54.04 \\
\hline 320 & 91.69 & 32.35 & 24.04 & 99.43 & 24.91 & 24.34 & 91.16 & 49.20 & 40.36 \\
\hline 321 & 88.66 & 53.25 & 41.90 & 99.34 & 47.67 & 47.01 & 88.07 & 75.53 & 63.61 \\
\hline 322 & 91.92 & 46.94 & 38.86 & 97.06 & 65.03 & 62.09 & 89.22 & 81.44 & 70.66 \\
\hline 323 & 87.46 & 37.02 & 24.48 & 96.52 & 69.93 & 66.46 & 84.42 & 81.06 & 65.49 \\
\hline 324 & 84.41 & 44.57 & 28.99 & 99.12 & 51.38 & 50.50 & 83.67 & 73.05 & 56.72 \\
\hline 325 & 91.41 & 29.14 & 20.54 & 99.32 & 50.24 & 49.56 & 90.79 & 64.74 & 55.52 \\
\hline 326 & 91.99 & 38.20 & 30.19 & 99.24 & 49.92 & 49.16 & 91.29 & 69.05 & 60.34 \\
\hline 327 & 91.04 & 41.89 & 32.92 & 95.59 & 65.30 & 60.89 & 87.02 & 79.84 & 66.85 \\
\hline
\end{tabular}

Table PITT-15 


\section{(\%) Кләлоэәу 'qmoว}

$\begin{array}{lllllllllll}00 \vdash & 06 & 08 & 0 L & 09 & 0 S & 0 t & 0 \varepsilon & 0 Z & 0 L & 0\end{array}$

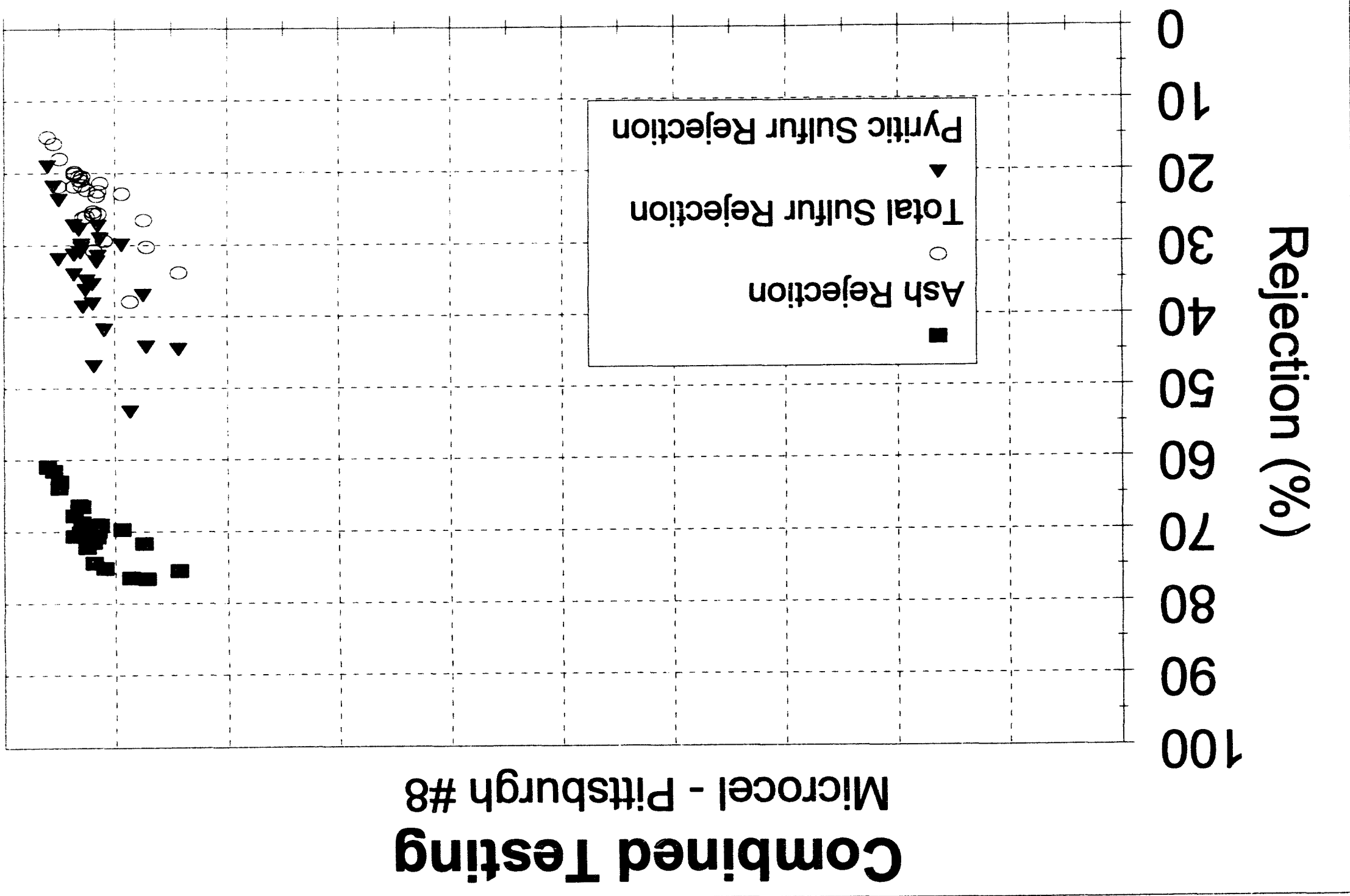




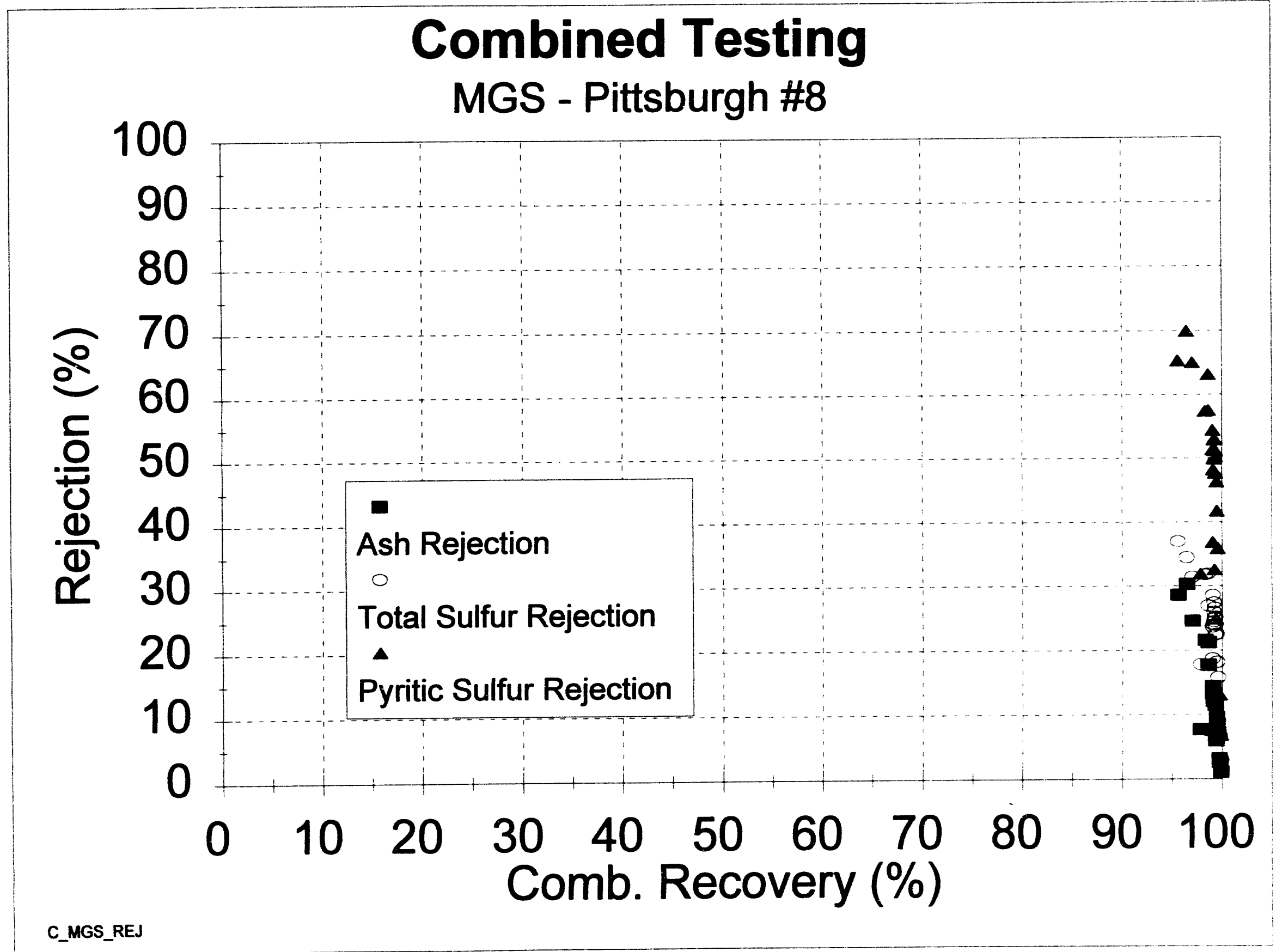

Figure PITT-4 


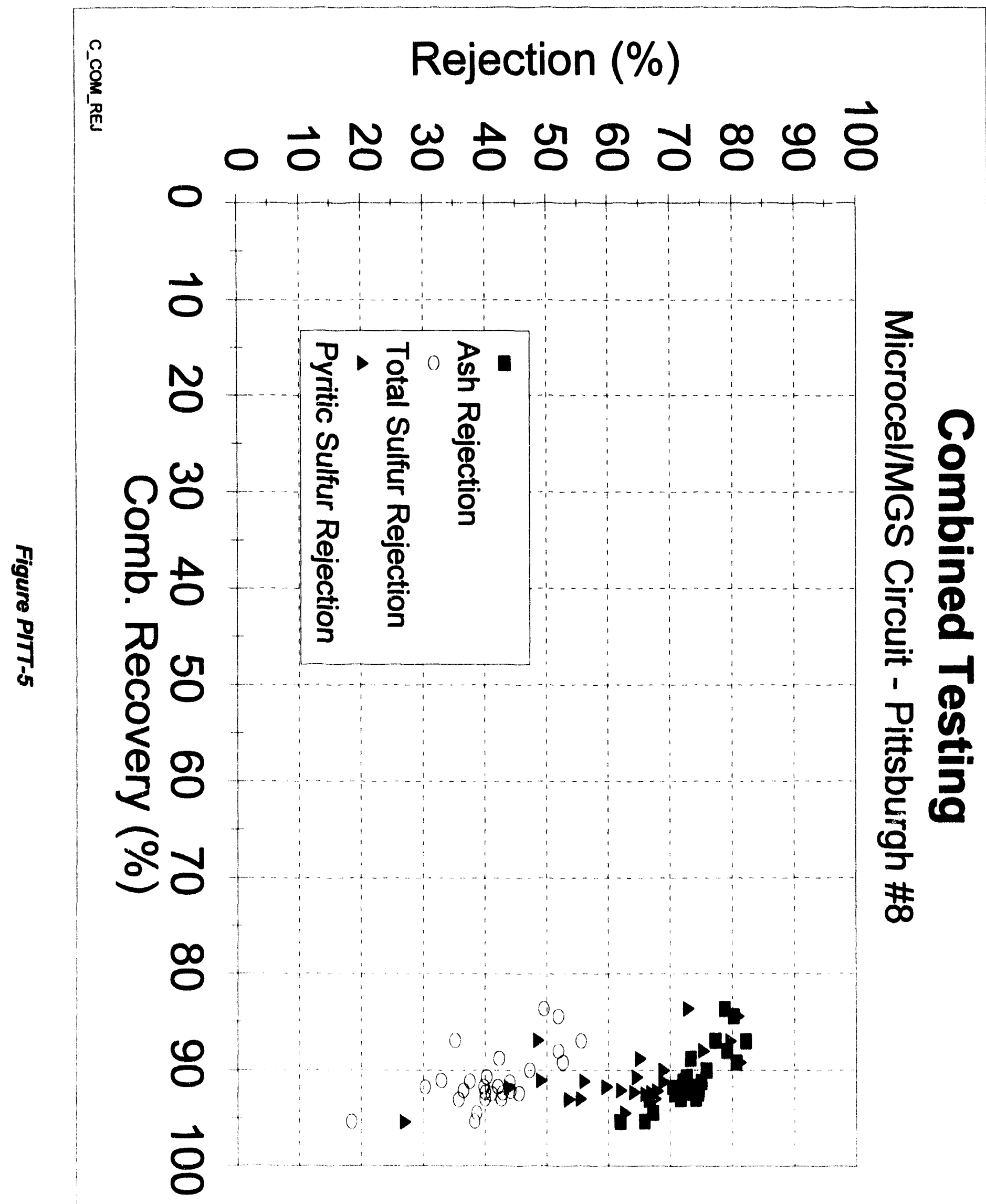




\section{Microcel/MGS Long Duration Testing - Pittsburgh \#8}

\begin{tabular}{lr}
\multicolumn{2}{c}{ Microcel } \\
Feed Rate (lbs/ton): & 500 \\
Wash Water (lpm): & 39 \\
Percent Solids (w/w): & $12-14$ \\
Grind Size (d80 (microns)): & 200 \\
Air Flow Rate (SCFM): & 8.89 \\
Diesel dosage (lbs/ton): & 1.00 \\
Frother Dosage (ml/min): & 0.80 \\
\multicolumn{1}{c}{ MGS } & \\
Feed Rate (lbs/hr): & 273 \\
Drum Speed (rpm): & 320 \\
Wash water (lpm): & $0.50-0.56$ \\
Percent Solids (w/w): & $19-20$ \\
Shake Amplitude (mm): & 15 \\
Shake Frequency (cps): & 5 \\
Tilt Angle (degrees): & 6
\end{tabular}




\section{Combined Microcel/MGS Tests Results - Pittsburgh $\$ 8$}

Long Duration Tests - Ash

\begin{tabular}{|c|c|c|c|c|c|c|c|c|c|c|c|c|}
\hline \multirow[b]{2}{*}{$\begin{array}{l}\text { Thime } \\
\text { hours }\end{array}$} & \multicolumn{6}{|c|}{ Microcel } & \multicolumn{6}{|c|}{ MGS } \\
\hline & $\begin{array}{l}\text { Chasured } \\
\text { Mcrocel } \\
\text { Feed } \\
\text { Ash } \\
\text { \% }\end{array}$ & $\begin{array}{l}\text { Adjusted } \\
\text { Microcel } \\
\text { Feed } \\
\text { Ash } \\
\%\end{array}$ & $\begin{array}{l}\text { Measured } \\
\text { Mlcrocel } \\
\text { Product } \\
\text { Ash } \\
\text { \% }\end{array}$ & $\begin{array}{c}\text { Adjusted } \\
\text { Mlcrocel } \\
\text { Product } \\
\text { Ash } \\
\text { \% }\end{array}$ & $\begin{array}{l}\text { Measured } \\
\text { Mllcrocel } \\
\text { Roject } \\
\text { Ash } \\
\text { \% }\end{array}$ & $\begin{array}{c}\text { Adjusted } \\
\text { Microcel } \\
\text { Reject } \\
\text { Ash } \\
\%\end{array}$ & $\begin{array}{l}\text { Mensured } \\
\text { Mos } \\
\text { Feed } \\
\text { Ash } \\
\text { \% }\end{array}$ & $\begin{array}{l}\text { Adjusted } \\
\text { mGs } \\
\text { Feed } \\
\text { Ash } \\
\%\end{array}$ & $\begin{array}{l}\text { Masured } \\
\text { Mos } \\
\text { Lights } \\
\text { Ash } \\
\text { \% }\end{array}$ & $\begin{array}{c}\text { Adjusted } \\
\text { ugs } \\
\text { Lights } \\
\text { Ash } \\
\%\end{array}$ & \begin{tabular}{|c|} 
Measured \\
ugs \\
Heavies \\
Ash \\
\%
\end{tabular} & $\begin{array}{l}\text { Adjusted } \\
\text { mGS } \\
\text { Heavies } \\
\text { Ash } \\
\% \\
\end{array}$ \\
\hline & & & & & & & & & & & & \\
\hline 1 & 19.84 & 19.78 & 7.01 & 6.98 & 69.04 & 69.20 & 6.92 & 6.98 & 5.56 & 5.54 & 34.03 & 33.99 \\
\hline 2 & 19.14 & 19.11 & 7.10 & 7.05 & 67.85 & 67.92 & 7.01 & 7.05 & 5.67 & 5.68 & 40.30 & 40.32 \\
\hline 3 & 19.11 & 19.21 & 7.12 & 7.16 & 70.63 & 70.37 & 7.26 & 7.16 & 5.75 & 5.78 & 35.81 & 35.86 \\
\hline 4 & 19.50 & 19.52 & 7.13 & 7.17 & 69.59 & 69.53 & 7.21 & 7.17 & 5.80 & 5.79 & 40.90 & 40.08 \\
\hline 5 & 19.33 & 19.14 & 7.59 & 7.39 & 67.52 & 67.98 & 7.25 & 7.39 & 6.00 & 6.05 & 42.34 & 42.43 \\
\hline 6 & 18.67 & 18.83 & 7.13 & 7.29 & 65.81 & 65.42 & 7.41 & 7.29 & 6.12 & 6.07 & 41.99 & 41.91 \\
\hline 7 & 19.11 & 19.39 & 6.99 & 7.17 & 66.26 & 65.56 & 7.49 & 7.17 & 5.67 & 5.71 & 40.33 & 40.43 \\
\hline 8 & 18.93 & 18.86 & 7.08 & 7.02 & 65.19 & 65.35 & 6.95 & 7.02 & 5.79 & 5.79 & 39.93 & 39.93 \\
\hline 9 & 18.69 & 18.64 & 7.43 & 7.35 & 67.75 & 67.86 & 7.32 & 7.35 & 5.88 & 5.92 & 40.73 & 40.80 \\
\hline
\end{tabular}

\begin{tabular}{|c|c|c|c|c|c|c|}
\hline \multirow[b]{2}{*}{$\begin{array}{l}\text { Time } \\
\text { hours }\end{array}$} & \multicolumn{6}{|c|}{ realds } \\
\hline & $\begin{array}{l}\text { Measured } \\
\text { Microcel } \\
\text { Ash } \\
\text { Yeld } \\
\text { \% }\end{array}$ & $\begin{array}{l}\text { Adjusted } \\
\text { Microcel } \\
\text { Ash } \\
\text { Yleld } \\
\%\end{array}$ & $\begin{array}{l}\text { Moasured } \\
\text { MGS } \\
\text { Ash } \\
\text { Yleld } \\
\%\end{array}$ & $\begin{array}{l}\text { Adjusted } \\
\text { MGs } \\
\text { Ash } \\
\text { Yleld } \\
\%\end{array}$ & $\begin{array}{c}\text { Coasured } \\
\text { Combined } \\
\text { Ash } \\
\text { Yield } \\
\text { \% }\end{array}$ & $\begin{array}{l}\text { Adjusted } \\
\text { Combined } \\
\text { Ash } \\
\text { Yield } \\
\%\end{array}$ \\
\hline & & & & & & \\
\hline 1 & 79.32 & 79.43 & 95.22 & 94.94 & 75.53 & 75.41 \\
\hline 2 & 80.18 & 80.19 & 96.13 & 96.05 & 77.08 & 77.02 \\
\hline 3 & 81.12 & 80.94 & 94.98 & 95.41 & 77.05 & 77.22 \\
\hline 4 & 80.20 & 80.20 & 95.98 & 95.98 & 76.97 & 76.97 \\
\hline 5 & 80.41 & 80.61 & 96.56 & 96.32 & 77.64 & 77.64 \\
\hline 6 & 80.33 & 80.15 & 96.40 & 96.60 & 77.44 & 77.42 \\
\hline 7 & 79.55 & 79.07 & 94.75 & 95.79 & 75.37 & 75.75 \\
\hline 8 & 79.61 & 79.70 & 96.60 & 96.40 & 76.90 & 76.83 \\
\hline 9 & 81.33 & 81.34 & 95.87 & 95.90 & 77.97 & 78.01 \\
\hline
\end{tabular}

Table PITT-17 
Combined Microcel/MGS Tests Results - Pittsburgh \#8

\section{Long Duration Tests - Total Sulfur}

\begin{tabular}{|c|c|c|c|c|c|c|c|c|c|c|c|c|}
\hline \multirow[b]{2}{*}{$\begin{array}{l}\text { Time } \\
\text { hours }\end{array}$} & \multicolumn{6}{|c|}{ Mlcroced } & \multicolumn{6}{|c|}{ MGS } \\
\hline & \begin{tabular}{c|} 
Measured \\
Microcel \\
Foed \\
T. Sulfur \\
$\%$
\end{tabular} & $\begin{array}{c}\text { Adjusted } \\
\text { microcel } \\
\text { Feed } \\
\text { T. Sulfur } \\
\%\end{array}$ & $\begin{array}{l}\text { Measured } \\
\text { Microcel } \\
\text { Product } \\
\text { T. Sulfur } \\
\%\end{array}$ & $\begin{array}{l}\text { Adjusted } \\
\text { Microcel } \\
\text { Product } \\
\text { T. Sulfur } \\
\%\end{array}$ & $\begin{array}{l}\text { Moasured } \\
\text { Microcel } \\
\text { Rejoct } \\
\text { T. Sulfur } \\
\text { \% }\end{array}$ & $\begin{array}{c}\text { Adjusted } \\
\text { Microcel } \\
\text { Reject } \\
\text { T. Sulfur } \\
\%\end{array}$ & \begin{tabular}{c|} 
mosasured \\
MGS \\
Feed \\
T. Sulfur \\
$\%$
\end{tabular} & $\begin{array}{l}\text { Adjusted } \\
\text { MGS } \\
\text { Feed } \\
\text { T. Sulfur } \\
\%\end{array}$ & $\begin{array}{c}\text { measured } \\
\text { mGS } \\
\text { Lights } \\
\text { T. Sulfur } \\
\%\end{array}$ & $\begin{array}{l}\text { Adjustied } \\
\text { MGS } \\
\text { Lights } \\
\text { T. Sulfur } \\
\text { \% }\end{array}$ & \begin{tabular}{c|} 
Measured \\
mGS \\
Heavies \\
T. Sulfur \\
$\%$
\end{tabular} & $\begin{array}{c}\text { Adjusted } \\
\text { mGS } \\
\text { Heavies } \\
\text { T. Sulfur } \\
\text { \% } \\
\end{array}$ \\
\hline & & & & & & & & & & & & \\
\hline 1 & 2.54 & 2.48 & 2.37 & 2.34 & 2.99 & 3.01 & 2.36 & 2.34 & 1.62 & 1.66 & 14.79 & 14.98 \\
\hline 2 & 2.56 & 2.49 & 2.29 & 2.34 & 3.06 & 3.08 & 2.38 & 2.34 & 1.71 & 1.73 & 17.32 & 17.38 \\
\hline 3 & 2.59 & 2.51 & 2.35 & 2.38 & 3.02 & 3.04 & 2.39 & 2.38 & 1.71 & 1.73 & 15.80 & 15.88 \\
\hline$\frac{4}{4}$ & 2.53 & 2.46 & 2.42 & 2.42 & 2.61 & 2.62 & 2.37 & 2.42 & 1.71 & 1.71 & \begin{tabular}{l|}
19.53 \\
\end{tabular} & 19.51 \\
\hline 5 & 2.47 & 2.44 & 2.40 & 2.36 & 2.77 & 2.78 & 2.32 & 2.36 & 1.68 & 1.69 & 20.03 & 20.06 \\
\hline$\frac{6}{6}$ & 2.44 & 2.38 & 2.34 & 2.32 & 2.59 & 2.60 & 2.33 & 2.32 & 1.68 & 1.72 & 19.30 & 19.47 \\
\hline 7 & 2.51 & 2.51 & 2.34 & 2.37 & 3.04 & 3.04 & 2.34 & 2.37 & 1.70 & 1.67 & 18.51 & 18.36 \\
\hline 8 & 2.39 & 2.42 & 2.38 & 2.30 & 2.90 & 2.89 & 2.31 & 2.30 & 1.68 & 1.72 & 17.70 & 17.85 \\
\hline$\frac{9}{9}$ & 2.42 & 2.42 & 2.36 & 2.41 & 2.46 & 2.46 & 2.41 & 2.41 & 1.72 & 1.70 & 18.95 & 18.85 \\
\hline
\end{tabular}


Combined MicrocelMGS Tests Results - Pittsburgh \#8

Long Duration Teats - Pyritic Sulfur

\begin{tabular}{|c|c|c|c|c|c|c|c|c|c|c|c|c|}
\hline \multirow[b]{2}{*}{$\begin{array}{l}\text { Time } \\
\text { hours }\end{array}$} & \multicolumn{6}{|c|}{ Microcel } & \multicolumn{6}{|c|}{ MGS } \\
\hline & \begin{tabular}{c|} 
Moasured \\
Microcel \\
Feed \\
Pyritic S \\
\%
\end{tabular} & $\begin{array}{l}\text { Adjusted } \\
\text { Microcel } \\
\text { Feed } \\
\text { Pyritic S } \\
\%\end{array}$ & $\begin{array}{l}\text { Deasured } \\
\text { Microcel } \\
\text { Product } \\
\text { Pyritic S } \\
\%\end{array}$ & $\begin{array}{c}\text { Adjustod } \\
\text { milcrocel } \\
\text { Product } \\
\text { Pyritic S } \\
\%\end{array}$ & \begin{tabular}{c|} 
Measured \\
Microcel \\
Reject \\
Pyritic S \\
$\%$
\end{tabular} & $\begin{array}{l}\text { Adjusted } \\
\text { microcel } \\
\text { Roject } \\
\text { Pyrtic S } \\
\% \\
\end{array}$ & $\begin{array}{c}\text { masured } \\
\text { mGS } \\
\text { Feed } \\
\text { Pyritic S } \\
\%\end{array}$ & $\begin{array}{l}\text { Adjusted } \\
\text { mGs } \\
\text { Foed } \\
\text { Pyritic S } \\
\%\end{array}$ & $\begin{array}{c}\text { mezaured } \\
\text { MGS } \\
\text { Lights } \\
\text { Pyritic S } \\
\%\end{array}$ & $\begin{array}{l}\text { Adjusted } \\
\text { mGS } \\
\text { Lights } \\
\text { Pyritic S } \\
\text { \% }\end{array}$ & $\begin{array}{l}\text { Measured } \\
\text { mGS } \\
\text { Heavies } \\
\text { Pyritic S } \\
\text { \% }\end{array}$ & $\begin{array}{l}\text { Adjusted } \\
\text { mGS } \\
\text { Heavies } \\
\text { Pyritic S } \\
\%\end{array}$ \\
\hline & & & & & & & & & & & & \\
\hline 1 & 1.33 & 1.26 & 1.03 & 1.01 & 2.16 & 2.20 & 0.96 & 1.01 & $\overline{0.41}$ & 0.41 & 12.30 & 12.27 \\
\hline 2 & 1.26 & 1.25 & 0.93 & 0.99 & 2.31 & 2.31 & 0.95 & 0.99 & 0.43 & 0.41 & 16.38 & 15.17 \\
\hline$\overline{3}$ & 1.27 & 1.38 & 0.94 & 1.02 & 3.00 & 2.89 & 1.02 & 1.02 & 0.42 & 0.40 & 15.70 & 14.02 \\
\hline$\overline{4}$ & 1.28 & 1.20 & 1.00 & 1.02 & 1.91 & 1.94 & 0.99 & 1.02 & 0.40 & 0.40 & 15.90 & 15.80 \\
\hline 5 & 1.22 & 1.21 & 1.04 & 1.01 & 2.04 & 2.04 & 0.91 & 1.01 & 0.43 & 0.41 & 17.68 & 16.62 \\
\hline$\frac{6}{6}$ & 1.18 & 1.16 & 0.95 & 0.98 & 1.87 & 1.88 & 1.06 & 0.98 & 0.41 & 0.42 & 16.70 & 17.09 \\
\hline$\frac{7}{7}$ & 1.34 & 1.26 & 0.94 & 1.00 & 2.19 & 2.24 & 0.88 & 1.00 & 0.44 & 0.41 & 16.57 & 14.51 \\
\hline 8 & 1.15 & 1.18 & 0.94 & 0.95 & 2.11 & 2.09 & 0.97 & 0.95 & 0.41 & 0.41 & 15.73 & 15.58 \\
\hline 9 & 1.13 & 1.17 & 1.03 & 1.03 & 1.78 & 1.76 & 0.97 & 1.03 & 0.42 & 0.40 & 16.94 & 15.78 \\
\hline
\end{tabular}




\section{Combined Microcel/MGS Tests Results - Pittsburgh \#8}

Long Duration Tests - Ash Performance

\begin{tabular}{|c|c|c|c|c|c|c|c|c|c|}
\hline \multirow[b]{2}{*}{$\begin{array}{l}\text { Time } \\
\text { hours }\end{array}$} & \multicolumn{3}{|c|}{ Dicrocel } & \multicolumn{3}{|c|}{ MGS } & \multicolumn{3}{|c|}{ Combined MicrocelligS } \\
\hline & $\begin{array}{c}\text { Comb. } \\
\text { Recovery } \\
\% \\
\end{array}$ & $\begin{array}{c}\text { Ash } \\
\text { Rejection } \\
\% \\
\end{array}$ & $\begin{array}{c}\text { Ash } \\
\text { Separation } \\
\text { Eficioncy } \\
\% \\
\end{array}$ & $\begin{array}{c}\text { Comb. } \\
\text { Recovery } \\
\% \\
\end{array}$ & $\begin{array}{c}\text { Ash } \\
\text { Rejection } \\
\% \\
\end{array}$ & \begin{tabular}{|c|} 
Ash \\
Separation \\
Efficiency \\
$\%$ \\
\end{tabular} & $\begin{array}{c}\text { Comb. } \\
\text { Recovery } \\
\% \\
\end{array}$ & $\begin{array}{c}\text { Ash } \\
\text { Rejection } \\
\% \\
\end{array}$ & \begin{tabular}{|c|} 
Ash \\
Separation \\
Efficiency \\
$\%$ \\
\end{tabular} \\
\hline & & & & & & & & & \\
\hline$\overline{1}$ & 92.10 & 71.97 & 64.07 & 96.41 & 24.65 & 21.06 & 88.79 & 78.88 & 67.67 \\
\hline 2 & 92.14 & 70.42 & 62.56 & 97.46 & 22.62 & 20.08 & 89.80 & 77.11 & 66.91 \\
\hline$\overline{3}$ & 93.01 & 69.83 & 62.84 & 96.83 & 22.98 & 19.81 & 90.06 & 76.76 & 66.83 \\
\hline$\overline{4}$ & 92.50 & 70.54 & 63.04 & 97.40 & 22.50 & 19.90 & 90.10 & 77.17 & 67.27 \\
\hline 5 & 92.32 & 68.88 & 61.20 & 97.71 & 21.15 & 18.86 & 90.21 & 75.46 & 65.67 \\
\hline$\overline{6}$ & 91.54 & 68.97 & 60.51 & 97.87 & 19.57 & 17.44 & 89.59 & 75.04 & 64.63 \\
\hline 7 & 91.06 & 70.76 & 61.82 & 97.30 & 23.71 & 21.01 & 88.60 & 77.69 & 66.30 \\
\hline$\overline{8}$ & 91.33 & 70.33 & 61.67 & 97.67 & 20.49 & 18.17 & 89.21 & 76.41 & 65.62 \\
\hline 9 & 92.63 & 67.93 & 60.56 & 97.38 & 22.76 & 20.14 & 90.20 & 75.23 & 65.43 \\
\hline
\end{tabular}




\section{Combined Microcel/MGS Tests Results - Pittsburgh \#8}

\section{Long Duration Tests - Total Sulfur Performance}

\begin{tabular}{|c|c|c|c|c|c|c|c|c|c|}
\hline \multirow[b]{2}{*}{$\begin{array}{l}\text { Time } \\
\text { hours }\end{array}$} & \multicolumn{3}{|c|}{ Microcel } & \multicolumn{3}{|c|}{ MGS } & \multicolumn{3}{|c|}{ Combined MicrocelhigS } \\
\hline & $\begin{array}{c}\text { Comb. } \\
\text { Recovery } \\
\%\end{array}$ & $\begin{array}{c}\text { T. Sulfur } \\
\text { Rejection } \\
\% \\
\end{array}$ & $\begin{array}{c}\text { T. Sulfur } \\
\text { Separation } \\
\text { Efficiency } \\
\%\end{array}$ & $\begin{array}{c}\text { Comb. } \\
\text { Recovery } \\
\% \\
\end{array}$ & $\begin{array}{c}\text { T. Suliur } \\
\text { Rejection } \\
\% \\
\end{array}$ & $\begin{array}{c}\text { T. Sulfur } \\
\text { Separation } \\
\text { Efficiency } \\
\%\end{array}$ & $\begin{array}{c}\text { Comb. } \\
\text { Recovery } \\
\% \\
\end{array}$ & $\begin{array}{c}\text { T. Sufiur } \\
\text { Rejection } \\
\% \\
\end{array}$ & $\begin{array}{c}\text { T. Sulfur } \\
\text { Separation } \\
\text { Efficiency } \\
\% \\
\end{array}$ \\
\hline & & & & & & & & & \\
\hline 1 & 92.10 & 25.06 & 17.16 & 96.41 & 32.65 & 29.06 & 88.79 & 49.53 & 38.32 \\
\hline 2 & 92.14 & 24.64 & 16.79 & 97.46 & 28.99 & 26.45 & 89.80 & 46.49 & 36.29 \\
\hline 3 & 93.01 & 23.26 & 16.26 & 96.83 & 30.65 & 27.48 & 90.06 & 46.77 & 36.83 \\
\hline 4 & 92.50 & 21.11 & 13.61 & 97.40 & 32.18 & 29.58 & 90.10 & 46.50 & 36.60 \\
\hline 5 & 92.32 & 22.04 & 14.36 & 97.71 & 31.03 & 28.74 & 90.21 & 46.23 & 36.43 \\
\hline 6 & 91.54 & 21.87 & 13.42 & 97.87 & 28.39 & 26.25 & 89.59 & 44.05 & 33.64 \\
\hline 7 & 91.06 & 25.34 & 16.40 & 97.30 & 32.50 & 29.80 & 88.60 & 49.60 & 38.20 \\
\hline 8 & 91.33 & 24.25 & 15.58 & 97.67 & 27.91 & 25.58 & 89.21 & 45.39 & 34.60 \\
\hline 9 & 92.63 & 18.99 & 11.62 & 97.38 & 32.35 & 29.73 & 90.20 & 45.20 & 35.40 \\
\hline
\end{tabular}




\section{Combined Microcel/MGS Tests Results - Pittsburgh \#8}

\section{Long Duration Tests - Pyritic Sulfur Performance}

\begin{tabular}{|r|c|c|c|c|c|c|c|c|c|}
\hline \multirow{2}{*}{$\begin{array}{c}\text { Time } \\
\text { hours }\end{array}$} & $\begin{array}{c}\text { Comb. } \\
\text { Recovery }\end{array}$ & $\begin{array}{c}\text { Pyritic S } \\
\text { Rejection }\end{array}$ & $\begin{array}{c}\text { Pyritic S } \\
\text { Separation } \\
\text { Efficiency } \\
\%\end{array}$ & $\begin{array}{c}\text { Comb. } \\
\text { Recovery }\end{array}$ & $\begin{array}{c}\text { Pyritic S } \\
\text { Rejection }\end{array}$ & $\begin{array}{c}\text { Pyritic S } \\
\text { Separation } \\
\text { Efficiency } \\
\%\end{array}$ & $\begin{array}{c}\text { Comb. } \\
\text { Recovery }\end{array}$ & $\begin{array}{c}\text { Pyritic S } \\
\text { Rejection }\end{array}$ & $\begin{array}{c}\text { Pyritic S } \\
\text { Separation } \\
\text { Efficiency } \\
\%\end{array}$ \\
\hline 1 & & & & & & & & & \\
\hline 2 & 92.10 & 36.33 & 28.43 & 96.41 & 61.46 & 57.87 & 88.79 & 75.46 & 64.26 \\
\hline 3 & 92.14 & 36.49 & 28.63 & 97.46 & 60.22 & 57.68 & 89.80 & 74.74 & 64.54 \\
\hline 4 & 93.01 & 40.18 & 33.19 & 96.83 & 62.58 & 59.41 & 90.06 & 77.62 & 67.68 \\
\hline 5 & 92.50 & 31.83 & 24.34 & 97.40 & 62.36 & 59.76 & 90.10 & 74.34 & 64.44 \\
\hline 6 & 92.32 & 32.72 & 25.04 & 97.71 & 60.90 & 58.61 & 90.21 & 73.69 & 63.90 \\
\hline 7 & 91.54 & 32.29 & 23.83 & 97.87 & 58.60 & 56.47 & 89.59 & 71.97 & 61.56 \\
\hline 8 & 91.06 & 37.24 & 28.30 & 97.30 & 60.72 & 58.03 & 88.60 & 75.35 & 63.95 \\
\hline 9 & 91.33 & 35.83 & 27.17 & 97.67 & 58.40 & 56.07 & 89.21 & 73.30 & 62.51 \\
\hline 92.63 & 28.39 & 21.02 & 97.38 & 62.76 & 60.14 & 90.20 & 73.33 & 63.53 \\
\hline
\end{tabular}




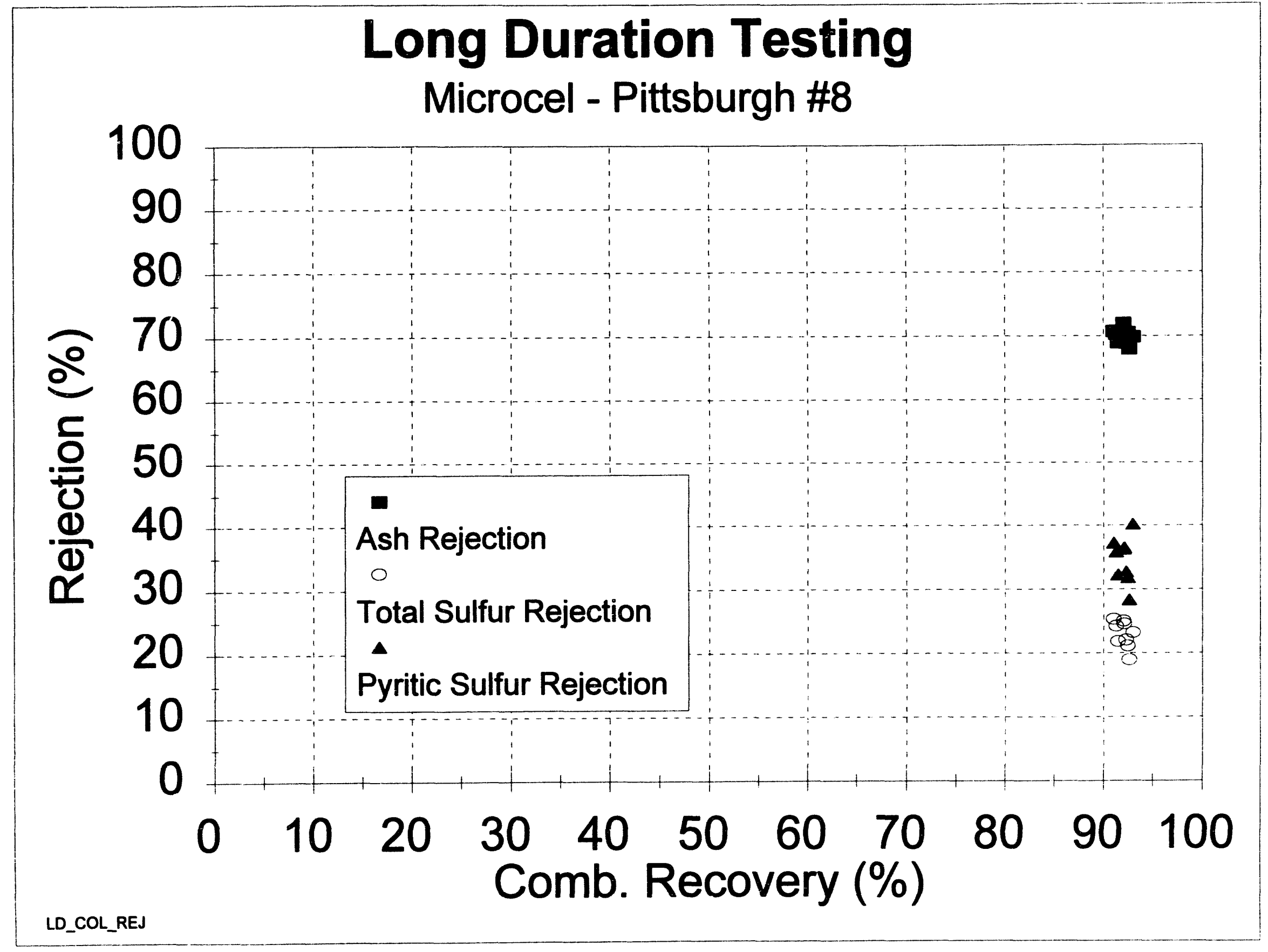

Figure PITT-6 


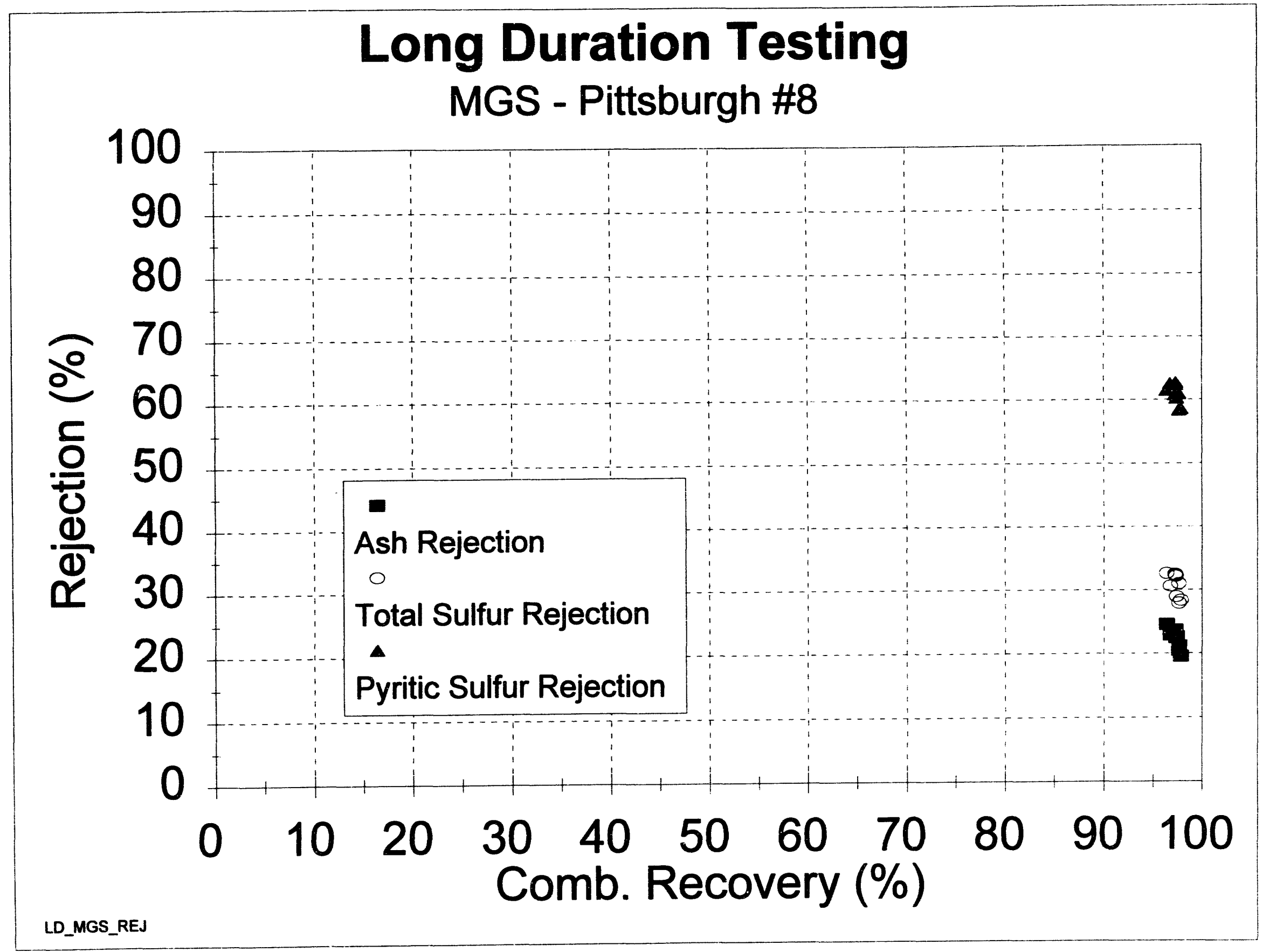

Figure PITT-7 


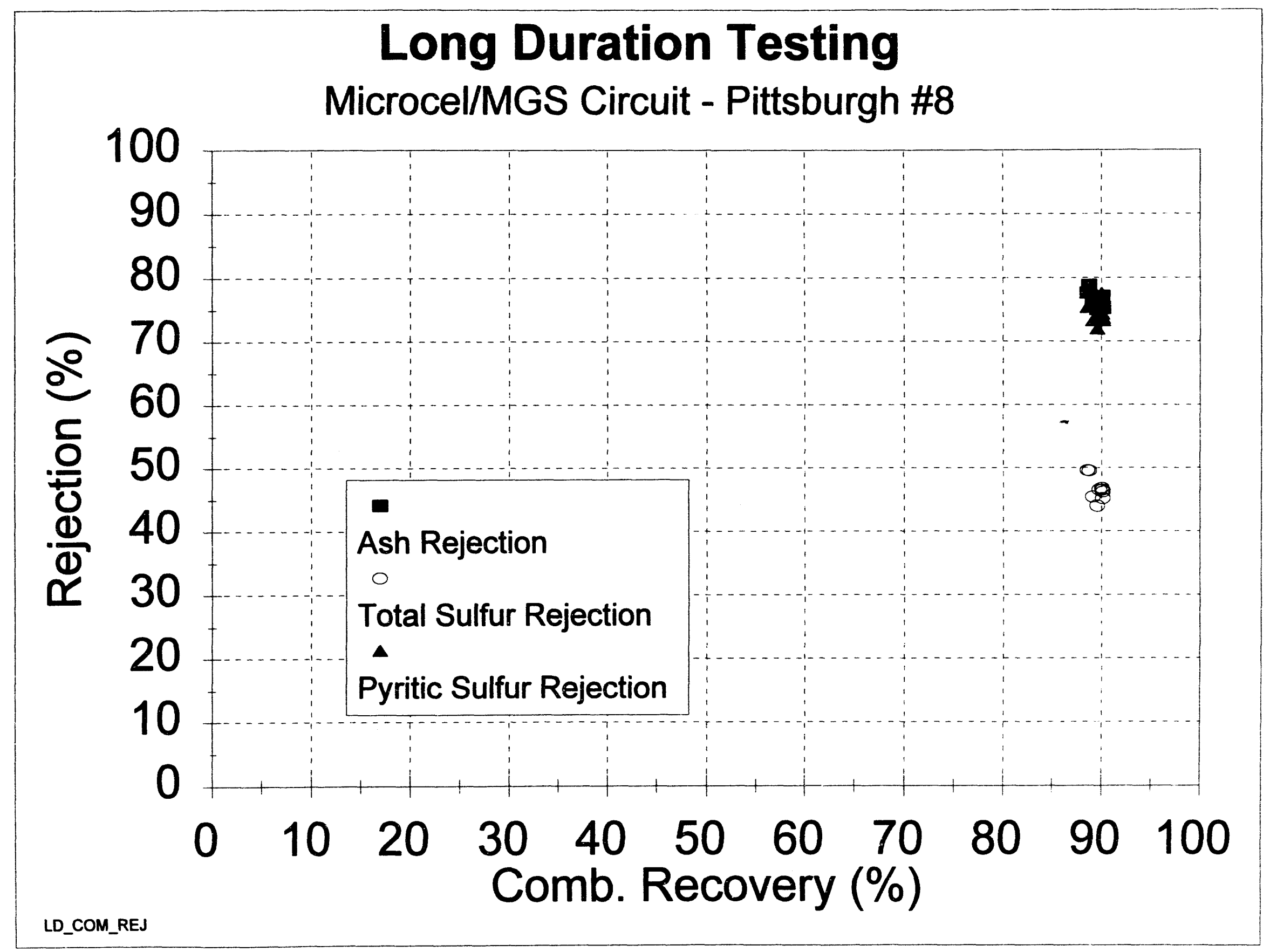

Figure PITT-8 
Microcel/MGS Independent Testing - Illinois \#6

Microcel Conditions Held Constant

$\begin{array}{lr}\text { Feed Rate (lbs/ton): } & 500 \\ \text { Wash Water (Ipm): } & 40.1 \\ \text { Percent Solids (w/w): } & 11-12 \\ \text { Grind Size (d80 (microns)): } & 200 \\ \text { Air Flow Rate (SCFM): } & 8.89\end{array}$

Microcel Collector and Frother Dosages

\begin{tabular}{||r|r||r|}
\hline $\begin{array}{c}\text { Test } \\
\text { Number }\end{array}$ & $\begin{array}{c}\text { Collector } \\
\text { Dosage } \\
\text { lbs/ton }\end{array}$ & $\begin{array}{c}\text { Frother } \\
\text { Dosage } \\
\text { ml/min }\end{array}$ \\
\hline 401 & 2.00 & 2.00 \\
\hline 402 & 2.00 & 1.00 \\
\hline 403 & 3.00 & 3.00 \\
\hline 404 & 2.00 & 3.00 \\
\hline 405 & 1.00 & 3.00 \\
\hline 406 & 1.00 & 2.00 \\
\hline 407 & 3.00 & 1.00 \\
\hline 408 & 3.00 & 2.00 \\
\hline 409 & 1.00 & 1.00 \\
\hline
\end{tabular}




\section{Microcel/MGS Independent Tests Results - Illinois \#6}

Microcel - Ash

\begin{tabular}{|c||c|c||c|r|r|r|r|r||}
\hline $\begin{array}{c}\text { Test } \\
\text { Number }\end{array}$ & $\begin{array}{c}\text { Measured } \\
\text { Column } \\
\text { Feed } \\
\text { Ash } \\
\%\end{array}$ & $\begin{array}{c}\text { Adjusted } \\
\text { Column } \\
\text { Feed } \\
\text { Ash } \\
\%\end{array}$ & $\begin{array}{c}\text { Measured } \\
\text { Column } \\
\text { Product } \\
\text { Ash } \\
\%\end{array}$ & $\begin{array}{c}\text { Adjusted } \\
\text { Column } \\
\text { Product } \\
\text { Ash } \\
\%\end{array}$ & $\begin{array}{c}\text { Measured } \\
\text { Column } \\
\text { Reject } \\
\text { Ash } \\
\%\end{array}$ & $\begin{array}{c}\text { Adjusted } \\
\text { Column } \\
\text { Reject } \\
\text { Ash } \\
\%\end{array}$ & $\begin{array}{c}\text { Measured } \\
\text { Column } \\
\text { Ash } \\
\text { Yield } \\
\%\end{array}$ & $\begin{array}{c}\text { Adjusted } \\
\text { Column } \\
\text { Ash } \\
\text { Yiold } \\
\%\end{array}$ \\
\hline 401 & 32.85 & 32.85 & 11.89 & 11.89 & 73.51 & 73.51 & 65.99 & 65.99 \\
\hline 402 & 39.52 & 39.56 & 9.53 & 9.53 & 74.00 & 73.94 & 53.48 & 53.38 \\
\hline 403 & 35.85 & 35.81 & 12.44 & 12.44 & 76.53 & 76.60 & 63.47 & 63.58 \\
\hline 404 & 34.26 & 34.19 & 12.17 & 12.18 & 75.20 & 75.32 & 64.95 & 65.14 \\
\hline 405 & 35.90 & 35.90 & 11.65 & 11.65 & 76.25 & 76.24 & 62.46 & 62.46 \\
\hline 406 & 32.23 & 32.38 & 12.40 & 12.38 & 76.37 & 76.10 & 69.00 & 68.61 \\
\hline 407 & 33.61 & 33.61 & 10.52 & 10.52 & 73.30 & 73.30 & 63.22 & 63.22 \\
\hline 408 & 34.37 & 34.32 & 10.20 & 10.20 & 78.12 & 78.22 & 64.41 & 64.54 \\
\hline 409 & 34.53 & 34.47 & 8.73 & 8.73 & 72.86 & 72.98 & 59.77 & 59.94 \\
\hline
\end{tabular}

\section{Microcel - Total Sulfur}

\begin{tabular}{|r|r|r|r|r|r|r|}
\hline $\begin{array}{c}\text { Test } \\
\text { Number }\end{array}$ & $\begin{array}{c}\text { Measured } \\
\text { Column } \\
\text { Feed } \\
\text { T. Sulfur } \\
\%\end{array}$ & $\begin{array}{c}\text { Adjusted } \\
\text { Column } \\
\text { Foed } \\
\text { T. Sulfur } \\
\%\end{array}$ & $\begin{array}{c}\text { Measured } \\
\text { Column } \\
\text { Product } \\
\text { T. Sulfur } \\
\%\end{array}$ & $\begin{array}{c}\text { Adjusted } \\
\text { Column } \\
\text { Product } \\
\text { T. Sulfur } \\
\%\end{array}$ & $\begin{array}{c}\text { Measured } \\
\text { Column } \\
\text { Reject } \\
\text { T. Sulfur } \\
\%\end{array}$ & $\begin{array}{c}\text { Adjusted } \\
\text { Column } \\
\text { Reject } \\
\text { T. Sulfur } \\
\%\end{array}$ \\
\hline 401 & 4.12 & 4.22 & 3.96 & 3.90 & 4.90 & 4.85 \\
\hline 402 & 4.77 & 4.36 & 3.88 & 4.02 & 4.58 & 4.75 \\
\hline 403 & 4.23 & 4.27 & 4.08 & 4.06 & 4.67 & 4.65 \\
\hline 404 & 4.17 & 4.60 & 4.42 & 4.11 & 5.81 & 5.52 \\
\hline 405 & 4.15 & 3.79 & 3.25 & 3.39 & 4.31 & 4.46 \\
\hline 406 & 4.13 & 4.29 & 4.24 & 4.12 & 4.74 & 4.67 \\
\hline 407 & 3.90 & 4.07 & 4.08 & 3.96 & 4.32 & 4.24 \\
\hline 408 & 4.07 & 4.30 & 3.70 & 3.58 & 5.76 & 5.60 \\
\hline 409 & 3.99 & 4.34 & 3.94 & 3.74 & 5.50 & 5.24 \\
\hline
\end{tabular}

Microcel - Pyritic Sulfur

\begin{tabular}{|r|r|r|r|r|r|r|}
\hline $\begin{array}{c}\text { Test } \\
\text { Number }\end{array}$ & $\begin{array}{c}\text { Measured } \\
\text { Column } \\
\text { Feed } \\
\text { Pyritic s } \\
\%\end{array}$ & $\begin{array}{c}\text { Adjusted } \\
\text { Column } \\
\text { Feed } \\
\text { Pyritic S } \\
\%\end{array}$ & $\begin{array}{c}\text { Measured } \\
\text { Column } \\
\text { Product } \\
\text { Pyritic 8 } \\
\%\end{array}$ & $\begin{array}{c}\text { Adjusted } \\
\text { Column } \\
\text { Product } \\
\text { Pyritic S } \\
\%\end{array}$ & $\begin{array}{c}\text { Measured } \\
\text { Column } \\
\text { Reject } \\
\text { Pyritic S } \\
\%\end{array}$ & $\begin{array}{c}\text { Adjusted } \\
\text { Column } \\
\text { Reject } \\
\text { Pyritic S } \\
\%\end{array}$ \\
\hline 401 & 2.58 & 2.62 & 1.91 & 1.90 & 4.06 & 4.03 \\
\hline 402 & 3.41 & 2.80 & 1.76 & 1.85 & 3.58 & 3.89 \\
\hline 403 & 2.40 & 2.58 & 2.01 & 1.93 & 3.87 & 3.70 \\
\hline 404 & 2.47 & 2.79 & 2.15 & 1.99 & 4.67 & 4.27 \\
\hline 405 & 2.65 & 2.61 & 1.93 & 1.94 & 3.69 & 3.72 \\
\hline 406 & 2.14 & 2.38 & 1.97 & 1.83 & 3.84 & 3.59 \\
\hline 407 & 2.10 & 2.32 & 1.93 & 1.82 & 3.38 & 3.18 \\
\hline 408 & 2.59 & 2.74 & 1.74 & 1.70 & 4.82 & 4.64 \\
\hline 409 & 2.31 & 2.62 & 1.72 & 1.62 & 4.64 & 4.13 \\
\hline
\end{tabular}




\section{Microcel/MGS Independent Tests Results - Illinois \#6}

Microcel Performance

\begin{tabular}{|r|r|r|r||r|r||r|r||}
\hline $\begin{array}{c}\text { Test } \\
\text { Number }\end{array}$ & $\begin{array}{c}\text { Comb. } \\
\text { Recovery }\end{array}$ & $\begin{array}{c}\text { Ash } \\
\text { Rejection }\end{array}$ & $\begin{array}{c}\text { Ash } \\
\text { Separation } \\
\text { Efficiency }\end{array}$ & $\begin{array}{c}\text { T. Sulfur } \\
\text { Rejection }\end{array}$ & $\begin{array}{c}\text { T. Sulfur } \\
\text { Separation } \\
\text { Efflciency }\end{array}$ & $\begin{array}{c}\text { Pyritic S } \\
\text { Rejection }\end{array}$ & $\begin{array}{c}\text { Pyritic S } \\
\text { Separation } \\
\text { Efficlency }\end{array}$ \\
\hline & $\%$ & $\%$ & $\%$ & $\%$ & $\%$ \\
\hline 401 & 86.58 & 76.12 & 62.70 & 39.02 & 25.60 & 52.15 & 38.73 \\
\hline 402 & 79.90 & 87.14 & 67.04 & 50.79 & 30.68 & 64.73 & 44.63 \\
\hline 403 & 86.72 & 77.91 & 64.64 & 39.55 & 26.27 & 52.44 & 39.16 \\
\hline 404 & 86.93 & 76.79 & 63.72 & 41.80 & 28.73 & 53.54 & 40.46 \\
\hline 405 & 86.08 & 79.73 & 65.82 & 44.14 & 30.22 & 53.58 & 39.66 \\
\hline 406 & 88.91 & 73.77 & 62.67 & 34.11 & 23.01 & 47.24 & 36.15 \\
\hline 407 & 85.21 & 80.21 & 65.42 & 38.49 & 23.70 & 50.40 & 35.61 \\
\hline 408 & 88.24 & 80.82 & 69.06 & 46.27 & 34.51 & 59.96 & 48.20 \\
\hline 409 & 83.48 & 84.82 & 68.30 & 48.35 & 31.83 & 62.94 & 46.42 \\
\hline
\end{tabular}




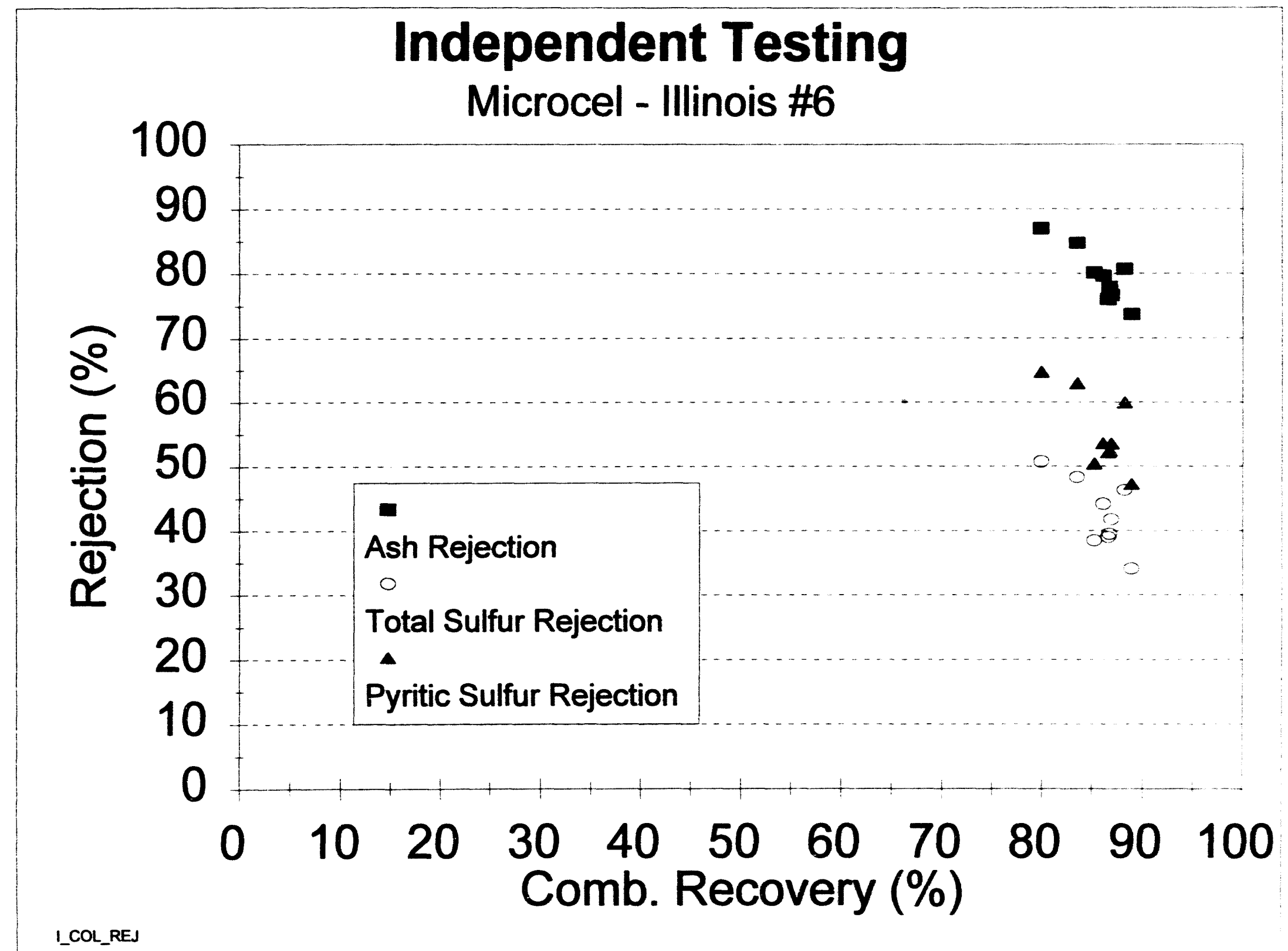

Figure ILL-1 


\title{
Microcel/MGS Independent Testing - Illinois \#6
}

\author{
MGS Conditions Held Constant \\ Shake Amplitude (mm): \\ Shake Frequency (cps): \\ 15 \\ Tilt Angle (degrees): \\ 5 \\ Grind Size (d80 (microns)): $\quad 200$
}

\section{MGS Variables}

\begin{tabular}{|c|c|c|c|c|}
\hline $\begin{array}{c}\text { Test } \\
\text { Number }\end{array}$ & $\begin{array}{c}\text { Drum } \\
\text { Speed } \\
\text { rpm } \\
\end{array}$ & $\begin{array}{l}\text { Wash } \\
\text { Water } \\
\text { Ipm }\end{array}$ & $\begin{array}{c}\text { Percent } \\
\text { Foed } \\
\text { Sollde } \\
\text { w/w } \\
\end{array}$ & $\begin{array}{l}\text { Feed } \\
\text { Rate } \\
\text { Ibe/hr }\end{array}$ \\
\hline & & & & \\
\hline 501 & $\frac{280}{n 00}$ & 0.76 & 30.71 & $\frac{345.21}{108.40}$ \\
\hline 502 & $\frac{280}{501}$ & 0.76 & 24.16 & 98.64 \\
\hline 503 & 281 & 1.63 & 34.63 & 199.60 \\
\hline 504 & 321 & 1.51 & 25.81 & 212.36 \\
\hline 505 & 280 & 0.00 & 18.95 & 234.40 \\
\hline 506 & 280 & 0.76 & 17.14 & 306.22 \\
\hline 507 & 281 & 0.80 & 29.60 & 241.91 \\
\hline 508 & 283 & 1.51 & 24.46 & 272.41 \\
\hline 509 & 280 & 0.00 & 26.32 & 293.41 \\
\hline 510 & 281 & 0.83 & 33.89 & 140.90 \\
\hline 511 & 283 & 0.68 & 25.56 & 210.43 \\
\hline 512 & 320 & 0.83 & 17.06 & 206.79 \\
\hline 513 & 241 & 1.55 & 26.40 & 216.30 \\
\hline 514 & 320 & 0.76 & 28.13 & 141.23 \\
\hline 515 & 320 & 0.80 & 37.77 & 229.75 \\
\hline 516 & 240 & 0.76 & 24.36 & 267.37 \\
\hline 517 & 280 & 0.00 & 30.11 & 154.62 \\
\hline 518 & 241 & 0.80 & 37.18 & 225.19 \\
\hline 519 & 320 & 0.76 & 25.38 & 279.12 \\
\hline 520 & 281 & 0.83 & 18.24 & 135.93 \\
\hline 521 & 280 & 1.59 & 28.63 & 148.15 \\
\hline 522 & 240 & 0.80 & 29.61 & 153.00 \\
\hline 523 & 241 & 0.83 & 17.22 & 209.50 \\
\hline 524 & 281 & 1.59 & 17.30 & 213.91 \\
\hline 52 & 322 & 0.00 & 25.72 & 216.83 \\
\hline 52 & 241 & 0.00 & 26.20 & 226.57 \\
\hline 52 & 28 & 0.00 & 37.75 & 260.0 \\
\hline
\end{tabular}




\section{Microcel/MGS Independent Tests Results - IIIInois \#6}

MGS - Ash

\begin{tabular}{|c|c|c|c|c|c|c|c|c|}
\hline $\begin{array}{c}\text { Teat } \\
\text { Number } \\
\end{array}$ & $\begin{array}{c}\text { Measured } \\
\text { Mos } \\
\text { Foed } \\
\text { Aah } \\
\% \\
\end{array}$ & $\begin{array}{c}\text { Adjusted } \\
\text { MGs } \\
\text { Feed } \\
\text { Ash } \\
\% \\
\end{array}$ & $\begin{array}{l}\text { Moasured } \\
\text { Mas } \\
\text { Lights } \\
\text { Ash } \\
\% \\
\end{array}$ & $\begin{array}{l}\text { Adjusted } \\
\text { Mos } \\
\text { Lights } \\
\text { Ash } \\
\% \\
\end{array}$ & $\begin{array}{c}\text { Measured } \\
\text { Mos } \\
\text { Heavies } \\
\text { Ash } \\
\% \\
\end{array}$ & $\begin{array}{c}\text { Adjusted } \\
\text { MGs } \\
\text { Heavies } \\
\text { Ash } \\
\% \\
\end{array}$ & $\begin{array}{c}\text { Measured } \\
\text { Mas } \\
\text { Ash } \\
\text { Ylold } \\
\% \\
\end{array}$ & $\begin{array}{c}\text { Adjueted } \\
\text { Mos } \\
\text { Ash } \\
\text { Ylold } \\
\%\end{array}$ \\
\hline & & & & & & & & \\
\hline 501 & 32.28 & 32.01 & 24.08 & 24.20 & 65.43 & 65.64 & 80.17 & 81.15 \\
\hline 602 & 35.28 & 35.54 & 24.07 & 23.98 & 68.64 & 68.39 & 74.85 & 73.97 \\
\hline 503 & 35.83 & 35.95 & 24.40 & 24.36 & 68.57 & 68.45 & 74.12 & 73.71 \\
\hline 504 & 32.61 & 32.93 & 22.13 & 22.02 & 65.79 & 65.46 & 76.00 & 74.88 \\
\hline 505 & 37.56 & 37.74 & 28.55 & 28.47 & 68.19 & 68.05 & 77.27 & 76.58 \\
\hline 506 & 36.14 & 35.17 & 29.76 & 30.34 & 69.59 & 70.03 & 83.98 & 87.83 \\
\hline 507 & 31.61 & 31.77 & 20.70 & 20.65 & 65.80 & 65.63 & 75.81 & 75.28 \\
\hline 508 & 32.18 & 32.24 & 23.05 & 23.02 & 68.55 & 68.49 & 78.93 & 79.72 \\
\hline 509 & 32.40 & 31.68 & 20.59 & 20.80 & 58.53 & 59.20 & 68.87 & 71.67 \\
\hline 510 & 33.53 & 33.92 & 24.56 & 24.40 & 65.36 & 65.01 & 78.01 & 76.56 \\
\hline 511 & 37.47 & 37.03 & 24.10 & 24.23 & 65.89 & 66.31 & 68.01 & 69.58 \\
\hline 512 & 36.43 & 36.09 & $27 . \overline{18}$ & 27.33 & 66.69 & 66.94 & 76.59 & 77.88 \\
\hline 513 & 33.33 & 33.33 & 26.24 & 26.24 & 75.33 & 75.33 & 85.56 & 85.56 \\
\hline 514 & 31.75 & 31.90 & 20.06 & 20.02 & 60.45 & 60.29 & 71.06 & 70.50 \\
\hline 515 & 34.55 & 35.00 & 23.86 & 23.71 & 61.98 & 61.55 & 71.96 & 70.16 \\
\hline 516 & 33.01 & 33.44 & 26.82 & 26.58 & 70.28 & 69.97 & 85.76 & 84.19 \\
\hline 517 & 34.65 & 34.69 & 20.79 & 20.78 & 58.39 & 58.35 & 63.14 & 62.98 \\
\hline 518 & 35.65 & 35.74 & 28.57 & 28.52 & 73.67 & 73.61 & 84.30 & 83.99 \\
\hline 519 & 31.47 & 31.51 & 20.76 & 20.75 & 60.49 & 60.45 & 73.04 & 72.90 \\
\hline 520 & 35.66 & 35.31 & 25.56 & 25.70 & 70.58 & 70.87 & 77.57 & 78.72 \\
\hline 521 & 36.83 & 37.02 & 24.80 & 24.74 & 70.34 & 70.15 & 73.58 & 72.96 \\
\hline 522 & 38.39 & 38.43 & 27.62 & 27.61 & 73.02 & 72.99 & 76.28 & 76.16 \\
\hline 523 & 39.76 & 39.70 & 36.26 & 37.03 & 72.02 & 72.04 & 90.21 & 92.37 \\
\hline 52 & 35.76 & 36.06 & 35.57 & 31.42 & 70.92 & 70.78 & 99.46 & 88.21 \\
\hline 52 & 36.78 & 36.23 & 24.29 & 24.45 & 58.76 & 59.23 & 63.77 & 66.13 \\
\hline 526 & 38.95 & 39.15 & 36.81 & 34.41 & 64.69 & 64.60 & 92.32 & 84.30 \\
\hline 5 & 8 & 13 & 25.27 & 5.29 & 61.08 & 61.12 & 73.72 & 73.93 \\
\hline
\end{tabular}




\section{Microcel/MGS Independent Tests Results - IIIInols \#6}

\section{MOS - Total Sulfur}

\begin{tabular}{|c|c|c|c|c|c|c|}
\hline $\begin{array}{c}\text { Test } \\
\text { Number }\end{array}$ & $\begin{array}{c}\text { Theasured } \\
\text { Mos } \\
\text { Foed } \\
\text { T. sulfur } \\
\% \\
\end{array}$ & $\begin{array}{l}\text { Adjuated } \\
\text { mos } \\
\text { Food } \\
\text { T. 8ulfur } \\
\% \\
\end{array}$ & $\begin{array}{c}\text { Mossured } \\
\text { Mos } \\
\text { Lights } \\
\text { T. Sulfur } \\
\% \\
\end{array}$ & $\begin{array}{l}\text { AdJunted } \\
\text { Mos } \\
\text { Lights } \\
\text { T. Sulfur } \\
\%\end{array}$ & $\begin{array}{c}\text { Theasured } \\
\text { MGs } \\
\text { Heavles } \\
\text { T. Sulfur } \\
\% \\
\end{array}$ & $\begin{array}{l}\text { Adjusted } \\
\text { Mos } \\
\text { Hoavies } \\
\text { T. 8ulfur } \\
\%\end{array}$ \\
\hline 501 & 3.65 & 3.53 & 2.43 & 2.471 & 797 & 80 \\
\hline 502 & 4.02 & 4.08 & 2.42 & 2.40 & 8.84 & $\frac{0.00}{8.86}$ \\
\hline 503 & 4.27 & 4.22 & 2.42 & 2.43 & 9.18 & 9.24 \\
\hline 504 & 4.01 & 3.92 & 2.43 & 2.46 & 8.19 & 8.29 \\
\hline 505 & 4.27 & 4.13 & 2.37 & 2.40 & 9.64 & 9.80 \\
\hline 506 & 3.87 & 3.60 & 2.48 & 2.59 & 10.70 & 10.95 \\
\hline 507 & 4.08 & 4.17 & 2.53 & 2.51 & 9.33 & 9.22 \\
\hline 508 & 4.00 & 3.99 & 2.56 & 2.56 & 8.60 & 9.61 \\
\hline 509 & 4.04 & 4.02 & 2.44 & 2.44 & 8.00 & 8.02 \\
\hline 510 & 3.68 & 3.76 & 2.30 & 2.28 & 8.69 & 8.59 \\
\hline 511 & 4.92 & 4.78 & 2.58 & 2.61 & 9.59 & 9.75 \\
\hline 512 & 4.27 & 4.08 & 2.45 & 2.50 & 9.45 & 9.65 \\
\hline 513 & 4.24 & 4.24 & 2.73 & 2.73 & 13.19 & 13.19 \\
\hline 514 & 4.19 & 4.16 & 2.53 & 2.54 & 8.03 & 8.06 \\
\hline 515 & 4.57 & 4.11 & 2.27 & 2.35 & 7.84 & 8.24 \\
\hline 516 & 4.21 & 4.20 & 2.66 & 2.66 & 12.35 & 12.37 \\
\hline 517 & 4.42 & 4.34 & 2.50 & 2.52 & 7.35 & 7.43 \\
\hline 518 & 4.38 & 4.34 & 2.54 & 2.55 & 13.67 & 13.73 \\
\hline 519 & 3.73 & 3.88 & 2.42 & 2.37 & 8.13 & \\
\hline 520 & 4.39 & 4.18 & 2.46 & 2.51 & 10.11 & 10. \\
\hline 521 & 4.78 & 4.73 & 2.56 & 2.57 & 10.48 & 10. \\
\hline 522 & 4.91 & 4.99 & 2.74 & 2.72 & 12.37 & 12.25 \\
\hline 523 & 4.26 & 3.89 & 2.67 & 2.81 & 16.54 & 16.97 \\
\hline 524 & 4.19 & 3.66 & 2.49 & 2.65 & 10.81 & 11.22 \\
\hline 525 & 3.85 & 3.8 & 2.29 & 2.27 & 7.25 & 7.16 \\
\hline 526 & 4.34 & 3.8 & 2.48 & 2. & 10.09 & 10.50 \\
\hline 527 & 4.28 & 4.02 & 2.44 & 2.50 & 8.06 & 8.3 \\
\hline
\end{tabular}


Microcel/MGs Independent Tests Results - IIIInols \#6

MOS - Pyritic Sulfur

\begin{tabular}{|c|c|c|c|c|c|c|}
\hline $\begin{array}{c}\text { Teat } \\
\text { Number }\end{array}$ & $\begin{array}{c}\text { Measurod } \\
\text { Mos } \\
\text { Foed } \\
\text { Pyritic } 8 \\
\% \\
\end{array}$ & $\begin{array}{l}\text { Adjuatod } \\
\text { Mos } \\
\text { Foed } \\
\text { Pyritle } 8 \\
\% \\
\end{array}$ & $\begin{array}{c}\text { Monsured } \\
\text { Mos } \\
\text { Llghts } \\
\text { Pyritic } 8 \\
\% \\
\end{array}$ & $\begin{array}{l}\text { Adjusted } \\
\text { Mos } \\
\text { Lights } \\
\text { Pyritic } 8 \\
\%\end{array}$ & $\begin{array}{c}\text { Moasured } \\
\text { MOs } \\
\text { Hoavios } \\
\text { Pyritic } 8 \\
\% \\
\end{array}$ & $\begin{array}{c}\text { Adjustod } \\
\text { Mas } \\
\text { Heavies } \\
\text { Pyritic } 8 \\
\% \\
\end{array}$ \\
\hline 501 & 488 & 137 & 0781 & 085 & 331 & \\
\hline 502 & 2.35 & 1.85 & $\frac{0.10}{0.85}$ & $\frac{0.00}{0.90}$ & $\frac{0.01}{4.14}$ & $\frac{5.01}{4.55}$ \\
\hline 503 & 2.58 & 1.78 & 0.76 & 0.81 & 4.00 & 4.50 \\
\hline 504 & 2.12 & 1.61 & 0.72 & 0.76 & 3.75 & 4.14 \\
\hline 505 & 2.34 & 1.76 & 0.86 & 0.92 & 4.08 & 4.48 \\
\hline 506 & 2.12 & 1.53 & 0.97 & 1.08 & 4.46 & 4.78 \\
\hline 507 & 2.19 & 1.50 & 0.74 & 0.80 & 3.27 & 3.65 \\
\hline 508 & 2.13 & 1.72 & 0.90 & 0.96 & 4.39 & 4.74 \\
\hline 509 & 7.90 & 1.52 & 0.73 & 0.77 & 3.14 & $3 . \overline{43}$ \\
\hline 510 & 1.95 & 7.56 & 0.74 & 0.78 & 3.76 & 4.10 \\
\hline 511 & 2.81 & 7.98 & 0.92 & 0.98 & 3.77 & 4.25 \\
\hline 512 & 2.61 & 1.73 & 0.89 & 0.97 & 3.98 & 4.43 \\
\hline 513 & 2.55 & 1.97 & 1.08 & 9.17 & 6.24 & 6.74 \\
\hline 514 & 2.09 & 1.48 & 0.64 & 0.68 & 3.05 & 3.43 \\
\hline 515 & 2.45 & 1.65 & 0.64 & 0.68 & 3.45 & 3.93 \\
\hline 516 & 2.32 & 1.85 & 1.01 & 1.08 & 5.53 & 5.95 \\
\hline 517 & 2.36 & 1.62 & 0.76 & 0.81 & 2.65 & 3.00 \\
\hline 518 & 2.43 & 1.88 & 1.00 & 1.08 & 5.60 & 6.07 \\
\hline 519 & 1.87 & 1.40 & 0.67 & 0.72 & 2.91 & 3.25 \\
\hline 520 & 2.62 & 1.73 & 0.80 & 0.87 & 4.39 & 4.92 \\
\hline 521 & 2.83 & 1.81 & 0.79 & 0.84 & 4.23 & 4.79 \\
\hline 522 & 2.78 & 2.11 & 0.92 & 0.98 & 5.18 & 5.73 \\
\hline 523 & 2.54 & 2.26 & 1.17 & 1.22 & 14.14 & 14.80 \\
\hline 524 & 2.61 & 2.27 & 1.13 & 1.19 & 9.79 & 10.36 \\
\hline 525 & 2.46 & 2.55 & 0.78 & 0.77 & 6.20 & 6.01 \\
\hline 52 & 2.98 & 2.59 & 1.16 & 1.21 & 9.41 & 10.02 \\
\hline & 2. & 2.37 & 0.72 & 0.7 & 6.73 & 7.0 \\
\hline
\end{tabular}




\section{Microcel/MGS Independent Tests Results - IIIinois \#6}

MGS Performance

\begin{tabular}{|c|c|c|c|c|c|c|c|}
\hline $\begin{array}{c}\text { Test } \\
\text { Number }\end{array}$ & $\begin{array}{l}\text { Comb. } \\
\text { Recovery }\end{array}$ & $\begin{array}{c}\begin{array}{c}\text { Ash } \\
\text { Rejection }\end{array} \\
\% \\
\end{array}$ & $\begin{array}{c}\text { Ash } \\
\text { Soparation } \\
\text { Efficioncy } \\
\% \\
\end{array}$ & $\begin{array}{c}\text { T.sulfur } \\
\text { Rejection } \\
\% \\
\end{array}$ & $\begin{array}{c}\text { T.suliur } \\
\text { Separation } \\
\text { Efficioncy } \\
\% \\
\end{array}$ & $\begin{array}{c}\text { Pyritic S } \\
\text { Rejection } \\
\%\end{array}$ & $\begin{array}{c}\text { Pyritic S } \\
\text { Soparation } \\
\text { Efficlency } \\
\% \\
\end{array}$ \\
\hline 501 & 90.48 & 38.65 & 29.12 & 43.22 & 33.69 & 49.65 & 40.12 \\
\hline$\frac{502}{502}$ & $\frac{8.40}{87.24}$ & 50.00 & 37.33 & $\frac{70.24}{56.49}$ & $\frac{30.00}{43.72}$ & $\frac{40.001}{64.01}$ & $\frac{40.12}{51.25}$ \\
\hline 503 & 87.05 & 50.05 & 37.10 & 57.55 & 44.61 & 66.46 & 53.51 \\
\hline 504 & 87.07 & 49.93 & 36.99 & 53.01 & 40.07 & 64.65 & 51.72 \\
\hline 505 & 87.98 & 42.23 & 30.21 & 55.50 & 43.48 & 59.97 & 47.95 \\
\hline 506 & 94.37 & 24.23 & 18.61 & 36.81 & 31.18 & 38.00 & 32.38 \\
\hline 507 & 87.55 & 51.07 & 38.62 & 54.69 & 42.24 & 59.85 & 47.40 \\
\hline 508 & 90.57 & 43.08 & 33.65 & 48.85 & 39.42 & 55.50 & 46.07 \\
\hline 509 & 83.08 & 52.95 & 36.03 & 56.50 & 39.58 & 63.70 & 46.77 \\
\hline 510 & 87.59 & 44.93 & 32.52 & 53.58 & 41.16 & 61.72 & 49.31 \\
\hline 511 & 83.73 & 54.47 & 38.20 & 62.01 & 45.73 & 65.56 & 49.29 \\
\hline 512 & 88.56 & 41.02 & 29.58 & 52.28 & 40.84 & 56.33 & 44.89 \\
\hline 513 & 94.66 & 32.64 & 27.30 & 44.91 & 39.57 & 49.19 & 43.84 \\
\hline 514 & 82.80 & 55.76 & 38.55 & 56.85 & 39.75 & 67.83 & 50.62 \\
\hline 515 & 82.35 & 52.47 & 34.82 & 59.88 & 42.23 & 71.08 & 53.43 \\
\hline 516 & 92.87 & 33.08 & 25.95 & 46.68 & 39.55 & 50.85 & 43.72 \\
\hline 517 & 76.39 & 62.28 & 38.66 & 63.43 & 39.82 & 68.51 & 44.90 \\
\hline 518 & 93.42 & 32.98 & 26.40 & 50.65 & 44.08 & 51.75 & 45.18 \\
\hline 519 & 84.35 & 52.00 & 36.35 & 55.47 & 39.82 & 62.51 & 46.86 \\
\hline 520 & 90.42 & 42.70 & 33.12 & 52.73 & 43.15 & 60.41 & 50.83 \\
\hline 521 & 87.18 & 51.24 & 38.43 & 60.36 & 47.54 & 67.91 & 55.10 \\
\hline 522 & 89.54 & 45.29 & 34.83 & 58.49 & 48.03 & 64.63 & 54.17 \\
\hline 523 & 96.46 & 13.84 & 10.30 & 33.27 & 29.74 & 50.13 & 46.60 \\
\hline 524 & 94.61 & 23.14 & 17.75 & 36.13 & 30.74 & 53.76 & 48.37 \\
\hline 525 & 78.35 & 55.37 & 33.72 & 61.80 & 40.15 & 80.03 & 58.38 \\
\hline 526 & 90.87 & 25.91 & 16.77 & 42.78 & 33.65 & 60.62 & 51.48 \\
\hline 527 & 84.50 & 46.01 & 30.50 & 54.02 & 38.52 & 77.23 & 61.72 \\
\hline
\end{tabular}




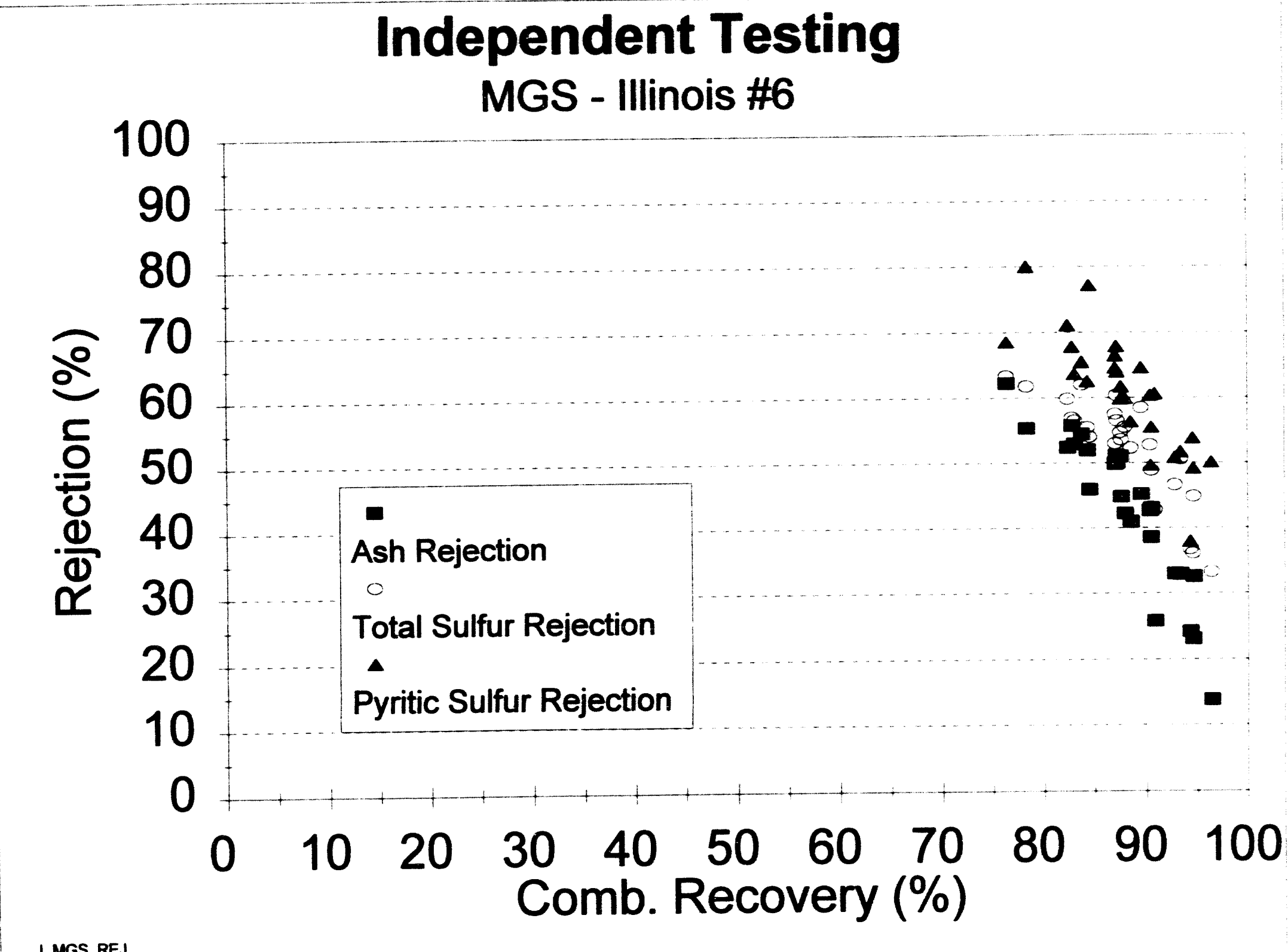

Figure ILL-2 


\section{MGS Conditions Held Constant}

$\begin{array}{lr}\text { Shake Amplitude (mm): } & 15 \\ \text { Shake Frequency (cps): } & 5 \\ \text { Tilt Angle (degrees): } & 6 \\ \text { Grind Size (d80 (microns) } & 200 \\ \text { Percent Solids (w/w): } & 21-22\end{array}$

MGS Variables

\begin{tabular}{|c|c|c|c|c|}
\hline $\begin{array}{c}\text { Test } \\
\text { Number }\end{array}$ & $\begin{array}{c}\text { Drum } \\
\text { Speed } \\
\text { rpm }\end{array}$ & $\begin{array}{l}\text { Wash } \\
\text { Water } \\
\text { Ipm }\end{array}$ & $\begin{array}{c}\text { Shako } \\
\text { Amplltude } \\
\text { mm }\end{array}$ & $\begin{array}{l}\text { Foed } \\
\text { Rate } \\
\text { lbs/hr }\end{array}$ \\
\hline & & & & \\
\hline 601 & 283 & 1.51 & 10 & 269.00 \\
\hline 602 & 283 & 0.95 & 15 & 269.00 \\
\hline 603 & 280 & 0.47 & 20 & 257.08 \\
\hline 604 & 239 & 1.08 & 15 & 341.50 \\
\hline 605 & 239 & 0.95 & 20 & 276.02 \\
\hline 606 & 280 & 0.95 & 15 & 267.90 \\
\hline 607 & 240 & 1.51 & 15 & 272.84 \\
\hline 608 & 280 & 1.51 & 15 & 160.99 \\
\hline 608 & 323 & 1.10 & 10 & 306.12 \\
\hline 610 & 239 & 0.45 & 15 & 259.45 \\
\hline 611 & 322 & 0.91 & 20 & 281.57 \\
\hline 612 & 322 & 0.98 & 15 & 148.13 \\
\hline 613 & 280 & 1.59 & 20 & 241.03 \\
\hline 614 & 282 & 0.95 & 15 & 299.91 \\
\hline 615 & 280 & 0.45 & 15 & 147.53 \\
\hline 616 & 321 & 1.06 & 15 & 342.89 \\
\hline 617 & 282 & 0.45 & 15 & 356.58 \\
\hline 618 & 282 & 0.93 & 20 & 377.79 \\
\hline 619 & 240 & 0.96 & 15 & 163.11 \\
\hline 620 & 282 & 1.51 & 15 & 352.70 \\
\hline 621 & 283 & 0.53 & 10 & 287.10 \\
\hline 622 & 241 & 1.06 & 10 & 219.17 \\
\hline 623 & 322 & 1.55 & 15 & 238.37 \\
\hline 624 & 281 & 1.10 & 10 & 160.41 \\
\hline 625 & 320 & 0.53 & 15 & 305.49 \\
\hline 626 & 280 & 0.98 & 20 & 134.70 \\
\hline 627 & 281 & 1.10 & 10 & 376.77 \\
\hline
\end{tabular}

\section{Microcel}

Feed Rate (Ibs/ton):

550.00

Wash Water (lpm):

40.1

Percent Solids (w/w):

Grind Size (d80 (microns)

$11-12$

Air Flow Rate (SCFM):

Diesel dosage (Ibs/ton):

Frother Dosage ( $\mathrm{ml} / \mathrm{min})$ : 
Combined Microcel/MGS Tests Results - Illinois \#6

Ash

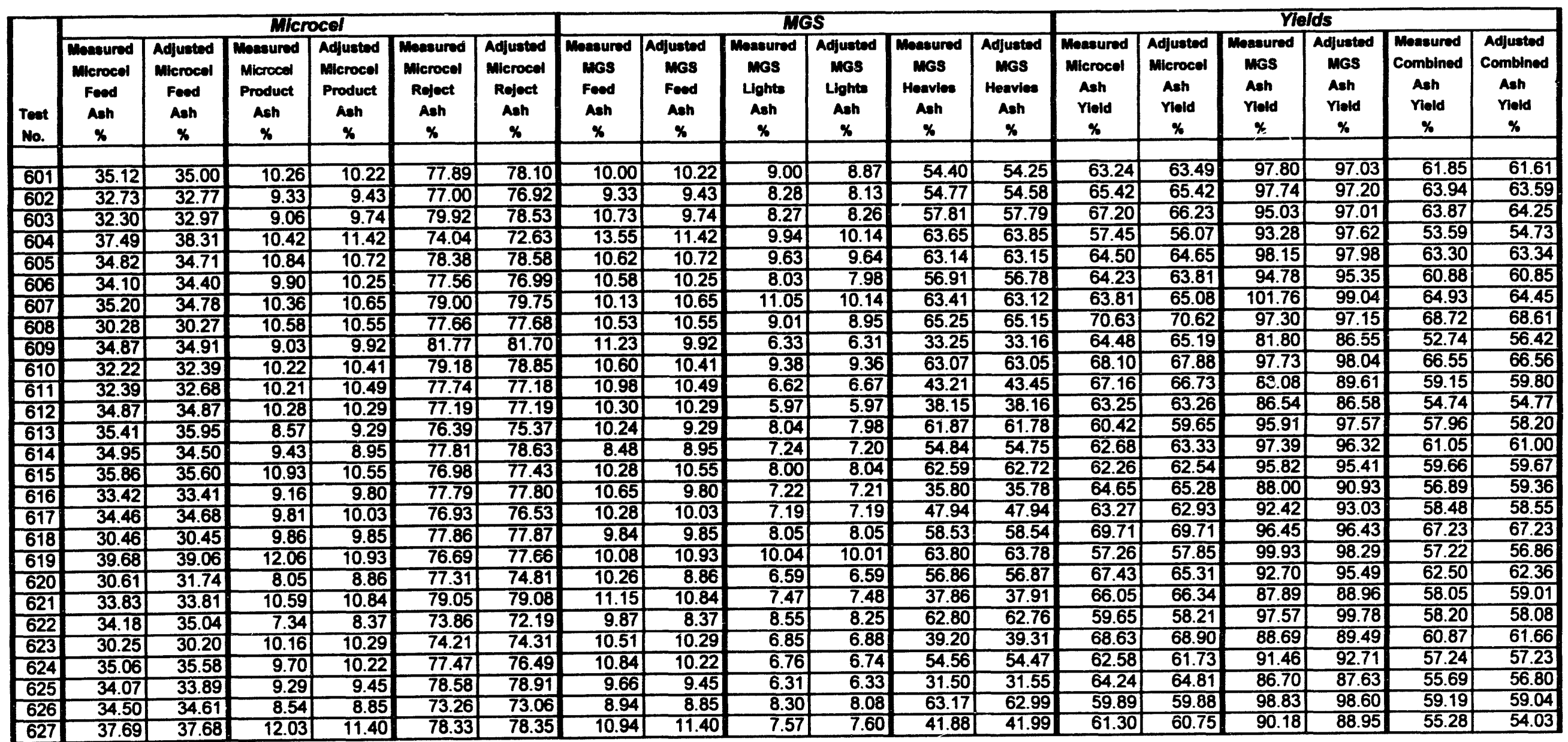




\section{Combined Microcel/MGS Tests Results - Illinois \#6 Total Sulfur}

\begin{tabular}{|c|c|c|c|c|c|c|c|c|c|c|c|c|}
\hline \multirow[b]{2}{*}{$\begin{array}{c}\text { Test } \\
\text { Number }\end{array}$} & \multicolumn{6}{|c|}{ Microcel } & \multicolumn{6}{|c|}{ MGS } \\
\hline & $\begin{array}{c}\text { Measured } \\
\text { Microcel } \\
\text { Feed } \\
\text { T. Sulfur } \\
\% \\
\end{array}$ & $\begin{array}{c}\text { Adjusted } \\
\text { Microcel } \\
\text { Feed } \\
\text { T. Sulfur } \\
\% \\
\end{array}$ & \begin{tabular}{|c|} 
Measured \\
Microcel \\
Product \\
T. Sulfur \\
$\%$ \\
\end{tabular} & $\begin{array}{c}\text { Adjusted } \\
\text { Microcel } \\
\text { Product } \\
\text { T. Sulfur } \\
\% \\
\end{array}$ & $\begin{array}{c}\text { Measured } \\
\text { Microcel } \\
\text { Reject } \\
\text { T. Sulfur } \\
\% \\
\end{array}$ & $\begin{array}{c}\text { Adjusted } \\
\text { Microcel } \\
\text { Reject } \\
\text { T. Sulfur } \\
\% \\
\end{array}$ & $\begin{array}{c}\text { Measured } \\
\text { MGS } \\
\text { Feed } \\
\text { T. Sulfur } \\
\% \\
\end{array}$ & $\begin{array}{c}\text { Adjusted } \\
\text { MGS } \\
\text { Feed } \\
\text { T. Sulfur } \\
\% \\
\end{array}$ & $\begin{array}{c}\text { Measured } \\
\text { MGS } \\
\text { Lights } \\
\text { T. Sulfur } \\
\% \\
\end{array}$ & $\begin{array}{c}\text { Adjusted } \\
\text { MGS } \\
\text { Lights } \\
\text { T. Sulfur } \\
\% \\
\end{array}$ & \begin{tabular}{|c|} 
Measured \\
MGS \\
Heavies \\
T. Sulfur \\
$\%$ \\
\end{tabular} & $\begin{array}{c}\text { Adjusted } \\
\text { MGS } \\
\text { Heavies } \\
\text { T. Sulfur } \\
\% \\
\end{array}$ \\
\hline & & & & & & & & & & & & \\
\hline 601 & 4.00 & 3.91 & 3.99 & 3.77 & 4.11 & 4.15 & 3.90 & 3.77 & 2.99 & 3.22 & 21.55 & 21.91 \\
\hline 602 & 4.06 & 3.88 & 3.88 & 3.73 & 4.11 & 4.17 & 3.88 & 3.73 & 2.85 & 3.07 & 26.29 & 26.82 \\
\hline 603 & 3.65 & 3.49 & 3.20 & 3.30 & 3.81 & 3.87 & 3.20 & 3.30 & 2.57 & 2.49 & 30.00 & 29.66 \\
\hline 604 & 4.30 & 4.62 & 4.05 & 4.20 & 5.37 & 5.15 & 4.01 & 4.20 & 3.64 & 3.24 & 45.15 & 43.64 \\
\hline 605 & 4.11 & 4.01 & 3.84 & 3.92 & 4.12 & 4.16 & 3.84 & 3.92 & 3.18 & 3.11 & 43.80 & 43.52 \\
\hline 606 & 3.82 & 3.85 & 3.55 & 3.66 & 4.22 & 4.20 & 3.84 & 3.66 & 2.46 & 2.48 & 27.70 & 27.80 \\
\hline 607 & 4.00 & 3.67 & 3.66 & 3.68 & 3.55 & 3.64 & 3.48 & 3.68 & 3.36 & 3.31 & 42.04 & 41.97 \\
\hline 608 & 3.29 & 3.54 & 3.67 & 3.60 & 3.47 & 3.39 & 3.76 & 3.60 & 2.68 & 2.69 & 34.50 & 34.54 \\
\hline 609 & 4.47 & 3.89 & 3.93 & 3.81 & 3.87 & 4.02 & 3.63 & 3.81 & 2.61 & 2.69 & 10.85 & 11.05 \\
\hline 610 & 3.76 & 3.66 & 3.68 & 3.74 & 3.47 & 3.50 & 3.80 & 3.74 & 2.95 & 2.99 & 41.10 & 41.26 \\
\hline 611 & 3.74 & 4.05 & 4.05 & 4.06 & 4.16 & 4.03 & 3.80 & 4.07 & 2.86 & 2.61 & 17.80 & 16.66 \\
\hline 612 & 4.09 & 4.12 & 4.00 & 3.83 & 4.64 & 4.62 & 3.76 & 3.83 & 2.23 & 2.25 & 14.00 & 14.10 \\
\hline 613 & 6.00 & 4.04 & 3.89 & 4.11 & 3.64 & 3.93 & 4.06 & 4.11 & 3.08 & 3.21 & 39.70 & 40.25 \\
\hline 614 & 4.11 & 3.40 & 3.91 & 3.52 & 3.06 & 3.20 & 3.19 & 3.52 & 2.51 & 2.63 & 26.40 & 26.90 \\
\hline 615 & 4.03 & 3.97 & 3.93 & 3.94 & 4.01 & 4.03 & 3.81 & 3.94 & 2.51 & 2.47 & 34.90 & 34.51 \\
\hline 616 & 3.85 & 4.04 & 3.77 & 3.83 & 4.54 & 4.45 & 4.12 & 3.83 & 2.73 & 2.76 & 14.48 & 14.56 \\
\hline 617 & 4.00 & 4.06 & 3.81 & 3.80 & 4.52 & 4.49 & 3.81 & 3.80 & 2.42 & 2.41 & 22.40 & 22.36 \\
\hline 618 & 3.80 & 3.78 & 3.75 & 3.71 & 3.91 & 3.92 & 3.70 & 3.71 & 2.64 & 2.66 & 32.20 & 32.30 \\
\hline 619 & 4.39 & 4.62 & 4.35 & 3.87 & 5.82 & 5.65 & 3.74 & 3.87 & 3.07 & 3.16 & 44.50 & 44.82 \\
\hline 620 & 3.59 & 3.81 & 3.63 & 3.90 & 3.70 & 3.62 & 4.85 & 3.90 & 2.83 & 2.90 & 25.00 & 25.24 \\
\hline 621 & 3.28 & 3.63 & 3.70 & 3.73 & 3.59 & 3.45 & 3.97 & 3.73 & 2.53 & 2.48 & 14.00 & 13.81 \\
\hline 622 & 3.80 & 3.70 & 3.32 & 3.39 & 4.03 & 4.09 & 3.88 & 3.39 & 3.02 & 3.31 & 38.96 & 39.07 \\
\hline 623 & \begin{tabular}{|l|l|}
3.73 \\
\end{tabular} & 4.18 & 4.04 & 3.95 & 4.92 & 4.68 & 4.07 & 3.95 & 2.67 & 2.61 & 15.59 & 15.35 \\
\hline 624 & 3.44 & 3.57 & 3.54 & 3.69 & 3.43 & 3.38 & 3.91 & 3.69 & 2.45 & 2.43 & 19.90 & 19.78 \\
\hline 625 & 3.40 & 4.07 & 3.48 & 3.47 & 5.87 & 5.17 & 3.66 & 3.47 & 2.43 & 2.31 & 12.10 & 11.68 \\
\hline 626 & 3.98 & 3.67 & 3.65 & 3.64 & 3.61 & 3.71 & 3.68 & 3.64 & 2.98 & 3.12 & 39.90 & 40.25 \\
\hline 627 & 3.99 & 4.11 & 3.80 & 3.94 & 4.41 & 4.35 & 3.80 & 3.94 & 2.59 & 2.44 & 16.80 & 16.04 \\
\hline
\end{tabular}




\section{Combined Microcel/MGS Tests Results - Illinois \#6 \\ Pyritic Sulfur}

\begin{tabular}{|c|c|c|c|c|c|c|c|c|c|c|c|c|}
\hline \multirow[b]{2}{*}{$\begin{array}{c}\text { Test } \\
\text { Number }\end{array}$} & \multicolumn{6}{|c|}{ Microcel } & \multicolumn{6}{|c|}{ MGS } \\
\hline & $\begin{array}{c}\text { Measured } \\
\text { Microcel } \\
\text { Feed } \\
\text { Pyritic S } \\
\% \\
\end{array}$ & $\begin{array}{c}\text { Adjusted } \\
\text { Microcel } \\
\text { Feed } \\
\text { Pyritic S } \\
\% \\
\end{array}$ & $\begin{array}{c}\text { Measured } \\
\text { Microcel } \\
\text { Product } \\
\text { Pyritic S } \\
\% \\
\end{array}$ & $\begin{array}{c}\text { Adjusted } \\
\text { Microcel } \\
\text { Product } \\
\text { Pyritic S } \\
\% \\
\end{array}$ & $\begin{array}{c}\text { Measured } \\
\text { Microcel } \\
\text { Reject } \\
\text { Pyritic S } \\
\%\end{array}$ & $\begin{array}{c}\text { Adjusted } \\
\text { Microcel } \\
\text { Reject } \\
\text { Pyritic S } \\
\%\end{array}$ & \begin{tabular}{|c|} 
Measured \\
MGS \\
Feed \\
Pyritic S \\
$\%$ \\
\end{tabular} & $\begin{array}{c}\text { Adjusted } \\
\text { MGS } \\
\text { Feed } \\
\text { Pyritic S } \\
\% \\
\end{array}$ & $\begin{array}{c}\text { Measured } \\
\text { MGS } \\
\text { Lights } \\
\text { Pyritic S } \\
\% \\
\end{array}$ & $\begin{array}{l}\text { Adjusted } \\
\text { MGS } \\
\text { Lights } \\
\text { Pyritic S } \\
\% \\
\end{array}$ & $\begin{array}{c}\text { Measured } \\
\text { MGS } \\
\text { Heavies } \\
\text { Pyritic S } \\
\% \\
\end{array}$ & $\begin{array}{c}\text { Adjusted } \\
\text { MGS } \\
\text { Heavies } \\
\text { Pyritic S } \\
\%\end{array}$ \\
\hline & & & & & & & & & & & & \\
\hline 601 & 1.96 & 1.98 & 1.65 & 1.43 & 2.97 & 2.95 & 1.65 & 1.43 & 0.75 & 0.84 & 18.91 & 20.62 \\
\hline 602 & 1.87 & 1.80 & 1.53 & 1.40 & 2.45 & 2.50 & 1.53 & 1.40 & 0.64 & 0.69 & 23.80 & 25.88 \\
\hline 603 & 2.33 & 2.16 & 1.58 & 1.69 & 3.00 & 3.09 & 1.88 & 1.69 & 0.82 & 0.84 & 28.52 & 29.25 \\
\hline 604 & 2.60 & 2.60 & 1.91 & 2.11 & 3.22 & 3.22 & 2.11 & 2.11 & 1.26 & 1.17 & 43.13 & 40.62 \\
\hline 605 & 2.82 & 2.67 & 2.13 & 2.09 & 3.63 & 3.72 & 1.97 & 2.09 & 1.29 & 1.27 & 42.39 & 42.00 \\
\hline 606 & 2.46 & 2.32 & 1.78 & 1.81 & 3.14 & 3.22 & 2.02 & 1.81 & 0.55 & 0.57 & 25.60 & 27.30 \\
\hline 607 & 2.33 & 2.17 & 1.49 & 1.64 & 3.05 & 3.15 & 1.67 & 1.64 & 1.33 & 1.26 & 41.80 & 41.15 \\
\hline 608 & 2.01 & 2.16 & 1.91 & 1.79 & 3.16 & 3.05 & 1.99 & 1.79 & 0.89 & 0.94 & 29.25 & 30.71 \\
\hline 609 & 2.85 & 2.36 & 1.70 & 1.77 & 3.25 & 3.47 & 2.00 & 1.77 & 0.58 & 0.60 & 8.57 & 9.29 \\
\hline 610 & 2.35 & 2.38 & 1.92 & 1.97 & 3.25 & 3.23 & 2.00 & 1.97 & 1.24 & 1.22 & 39.92 & 39.57 \\
\hline 611 & 2.06 & 2.37 & 1.87 & 2.01 & 3.35 & 3.08 & 2.08 & 2.01 & 0.68 & 0.65 & 15.50 & 13.72 \\
\hline 612 & 2.90 & 2.29 & 2.16 & 2.12 & 2.43 & 2.59 & 1.93 & 2.12 & 0.55 & 0.55 & 12.27 & 12.29 \\
\hline 613 & 4.18 & 2.37 & 1.68 & 1.88 & 2.78 & 3.10 & 1.96 & 1.88 & 0.97 & 0.98 & 37.60 & 38.01 \\
\hline 614 & 3.07 & 2.23 & 1.77 & 1.67 & 2.92 & 3.20 & 1.57 & 1.67 & 0.76 & 0.79 & 23.70 & 24.72 \\
\hline 615 & 2.62 & 2.73 & 2.28 & 2.17 & 3.75 & 3.66 & 1.99 & 2.17 & 0.85 & 0.83 & 31.90 & 30.22 \\
\hline 616 & 2.26 & 2.22 & 1.54 & 1.70 & 3.16 & 3.19 & 1.94 & 1.70 & 0.67 & 0.67 & 11.97 & 12.01 \\
\hline 617 & 2.75 & 2.75 & 1.88 & 1.97 & 4.08 & 4.08 & 1.96 & 1.97 & 0.77 & 0.75 & 18.90 & 18.20 \\
\hline 618 & 2.17 & 2.26 & 1.87 & 1.79 & 3.39 & 3.33 & 1.76 & 1.79 & 0.90 & 0.90 & 26.00 & 25.99 \\
\hline 619 & 3.11 & 3.19 & 2.36 & 2.14 & 4.71 & 4.63 & 1.99 & 2.14 & 1.44 & 1.43 & 42.80 & 42.71 \\
\hline 620 & 1.89 & 2.01 & 1.44 & 1.63 & 2.82 & 2.73 & 1.87 & 1.63 & 0.69 & 0.67 & 22.90 & 21.89 \\
\hline 621 & 1.84 & 2.20 & 2.01 & 1.90 & 3.15 & 2.80 & 2.01 & 1.90 & 0.65 & 0.64 & 12.24 & 12.00 \\
\hline 622 & 2.01 & 2.04 & 1.25 & 1.21 & 3.24 & 3.20 & 1.71 & 1.21 & 0.95 & 1.12 & 39.00 & 39.66 \\
\hline 623 & 1.82 & 2.21 & 1.80 & 1.73 & 3.79 & 3.26 & 1.64 & 1.73 & 0.64 & 0.61 & 13.03 & 11.33 \\
\hline 624 & 2.35 & 2.29 & 1.86 & 1.85 & 2.96 & 3.00 & 2.02 & 1.85 & 0.57 & 0.59 & 16.80 & 17.90 \\
\hline 625 & 2.20 & 2.62 & 1.76 & 1.70 & 5.13 & 4.32 & 1.92 & 1.70 & 0.57 & C.58 & 9.46 & 9.68 \\
\hline 626 & 2.18 & 1.98 & 1.47 & 1.49 & 2.61 & 2.72 & 1.43 & 1.49 & 0.99 & 0.98 & 38.25 & 37.95 \\
\hline 627 & 2.43 & 2.57 & 2.23 & 2.16 & 3.32 & 3.21 & 2.18 & 2.16 & 0.64 & 0.64 & 14.30 & 14.39 \\
\hline
\end{tabular}

Table ILL-12 


\section{Combined Microcel/MGS Tests Results - Illinois \#6 Ash Performance}

\begin{tabular}{|c|c|c|c|c|c|c|c|c|c|}
\hline $\begin{array}{c}\text { Test } \\
\text { Number }\end{array}$ & $\begin{array}{c}\text { Comb. } \\
\text { Recovery } \\
\% \\
\end{array}$ & $\begin{array}{c}\text { Microcel } \\
\text { Ash } \\
\text { Rejection } \\
\% \\
\end{array}$ & $\begin{array}{c}\text { Ash } \\
\text { Separation } \\
\text { Efficiency } \\
\% \\
\end{array}$ & \begin{tabular}{|c|} 
Comb. \\
Recovery \\
$\%$ \\
\end{tabular} & $\begin{array}{c}\text { MGS } \\
\text { Ash } \\
\text { Rejection } \\
\% \\
\end{array}$ & $\begin{array}{c}\text { Ash } \\
\text { Separation } \\
\text { Efficiency } \\
\% \\
\end{array}$ & \begin{tabular}{c|} 
Comb \\
Comb. \\
Recovery \\
$\%$ \\
\end{tabular} & \begin{tabular}{c|} 
ined Microc \\
Ash \\
Rejection \\
$\%$ \\
\end{tabular} & $\begin{array}{c}\text { MGGS } \\
\text { Ash } \\
\text { Separation } \\
\text { Efficiency } \\
\% \\
\end{array}$ \\
\hline & & & & & & & & & \\
\hline 601 & 87.70 & 81.46 & 69.16 & 98.48 & 15.79 & 14.28 & 86.37 & 84.39 & 70.76 \\
\hline 602 & 88.13 & 81.18 & 69.30 & 98.60 & 16.20 & 14.80 & 86.89 & 84.22 & 71.12 \\
\hline 603 & 89.18 & 80.43 & 69.62 & 98.60 & 17.73 & 16.33 & 87.94 & 83.90 & 71.84 \\
\hline 604 & 80.51 & 83.29 & 63.80 & 99.03 & 13.32 & 12.35 & 79.73 & 85.51 & 65.24 \\
\hline 605 & 88.40 & 80.03 & 68.44 & 99.17 & 11.89 & 11.06 & 87.67 & 82.41 & 70.07 \\
\hline 606 & 87.31 & 80.99 & 68.29 & 97.76 & 25.77 & 23.53 & 85.35 & 85.89 & 71.24 \\
\hline 607 & 89.16 & 80.07 & 69.23 & 99.60 & 5.71 & 5.31 & 88.80 & 81.21 & 70.01 \\
\hline 608 & 90.60 & 75.39 & 65.98 & 98.89 & 17.58 & 16.47 & 89.59 & 79.71 & 69.31 \\
\hline 609 & 90.21 & 81.48 & 71.69 & 90.02 & 44.94 & 34.97 & 81.21 & 89.80 & 71.01 \\
\hline 610 & 89.95 & 78.18 & 68.14 & 99.19 & 11.84 & 11.04 & 89.23 & 80.77 & 69.99 \\
\hline 611 & 88.72 & 78.58 & 67.30 & 93.44 & 43.02 & 36.46 & 82.90 & 87.80 & 70.70 \\
\hline 612 & 87.13 & 81.33 & 68.46 & 90.75 & 49.77 & 40.52 & 79.07 & 90.62 & 69.69 \\
\hline 613 & 84.49 & 84.58 & 69.07 & 98.97 & 16.19 & 15.17 & 83.62 & 87.08 & 70.70 \\
\hline 614 & 88.04 & 83.57 & 71.61 & 98.17 & 22.51 & 20.68 & 86.43 & 87.27 & 73.70 \\
\hline 615 & 86.87 & 81.46 & 68.34 & 98.09 & 27.29 & 25.38 & 85.21 & 86.52 & 74.73 \\
\hline 616 & 88.42 & 80.85 & 69.28 & 93.55 & 33.10 & 26.64 & 82.72 & 87.19 & 69.91 \\
\hline 617 & 86.68 & 81.80 & 68.48 & 95.97 & 33.31 & 29.28 & 83.19 & 87.86 & 71.05 \\
\hline 618 & 90.36 & 77.45 & 67.81 & 98.36 & 21.19 & 19.55 & 88.88 & 82.23 & 71.11 \\
\hline 619 & 84.55 & 83.81 & 68.36 & 99.30 & 9.98 & 9.29 & 83.96 & 85.43 & 69.39 \\
\hline 620 & 87.20 & 81.77 & 68.97 & 97.86 & 28.98 & 26.84 & 85.33 & 87.05 & 72.39 \\
\hline 621 & 89.36 & 78.73 & 68.09 & 92.31 & 38.62 & 30.93 & 82.49 & 86.94 & 69.43 \\
\hline 622 & 82.11 & 86.10 & 68.20 & 99.91 & 1.65 & 1.56 & 82.04 & 86.32 & 68.36 \\
\hline 623 & 88.55 & 76.52 & 65.08 & 92.89 & 40.17 & 33.06 & 82.25 & 85.95 & 68.21 \\
\hline 624 & 86.03 & 82.27 & 68.30 & 96.30 & 38.86 & 35.16 & 82.85 & 89.16 & 72.01 \\
\hline 625 & 88.78 & 81.93 & 70.70 & 90.65 & 41.30 & 31.95 & 80.47 & 89.39 & 69.86 \\
\hline 626 & 83.47 & 84.69 & 68.16 & 99.43 & 9.98 & 9.41 & 83.00 & 86.22 & 69.21 \\
\hline 627 & 86.36 & 81.62 & 67.98 & 92.77 & 40.70 & 33.47 & 80.12 & 89.10 & 69.22 \\
\hline
\end{tabular}

Table ILL-13 


\section{Combined Microcel/MGS Tests Results - Illinois \#6 \\ Total Sulfur Performance}

\begin{tabular}{|c|c|c|c|c|c|c|c|c|c|}
\hline $\begin{array}{l}\text { Test } \\
\text { Number }\end{array}$ & \begin{tabular}{|c|} 
Comb. \\
Recovery \\
$\%$ \\
\end{tabular} & $\begin{array}{c}\text { Microcel } \\
\text { T. Sulfur } \\
\text { Rejection } \\
\% \\
\end{array}$ & $\begin{array}{c}\text { T. Sulfur } \\
\text { Separation } \\
\text { Efficiency } \\
\% \\
\end{array}$ & $\begin{array}{c}\text { Comb. } \\
\text { Recovery } \\
\% \\
\end{array}$ & $\begin{array}{c}\text { MGS } \\
\text { T. Sulfur } \\
\text { Rejection } \\
\% \\
\end{array}$ & $\begin{array}{c}\text { T. Sulfur } \\
\text { Separation } \\
\text { Efficiency } \\
\% \\
\end{array}$ & \begin{tabular}{|c|} 
Combin \\
Comb. \\
Recovery \\
$\%$ \\
\end{tabular} & $\begin{array}{c}\text { Microcel/ } \\
\text { T. Sulfur } \\
\text { Rejection } \\
\% \\
\end{array}$ & $\begin{array}{c}\text { GS } \\
\text { T. Sulfur } \\
\text { Separation } \\
\text { Efficiency } \\
\% \\
\end{array}$ \\
\hline & & & & & & & & & \\
\hline 601 & 87.70 & 38.78 & 26.48 & 98.48 & 17.13 & 15.61 & 86.37 & 49.27 & 35.64 \\
\hline 602 & 88.13 & 37.11 & 25.24 & 98.60 & 20.00 & 18.59 & 86.89 & 49.69 & 36.58 \\
\hline 603 & 89.18 & 37.38 & 26.56 & 98.60 & 26.80 & 25.40 & 87.94 & 54.16 & 42.10 \\
\hline 604 & 80.51 & 49.03 & 29.54 & 99.03 & 24.70 & 23.72 & 79.73 & 61.62 & 41.34 \\
\hline 605 & 88.40 & 36.80 & 25.20 & 99.17 & 22.26 & 21.43 & 87.67 & 50.87 & 38.54 \\
\hline 606 & 87.31 & 39.33 & 26.64 & 97.76 & 35.39 & 33.15 & 85.35 & 60.81 & 46.16 \\
\hline 607 & 89.16 & 34.74 & 23.90 & 99.60 & 10.92 & 10.52 & 88.80 & 41.87 & 30.67 \\
\hline 608 & 90.60 & 28.18 & 18.78 & 98.89 & 27.41 & 26.30 & 89.59 & 47.86 & 37.45 \\
\hline 609 & 90.21 & 36.16 & 26.37 & 90.02 & 38.89 & 28.91 & 81.21 & 60.98 & 42.20 \\
\hline 610 & 89.95 & 30.63 & 20.59 & 99.19 & 21.62 & 20.81 & 89.23 & 45.63 & 34.86 \\
\hline 611 & 88.72 & 33.11 & 21.83 & 93.44 & 42.53 & 35.97 & 82.90 & 61.46 & 44.36 \\
\hline 612 & 87.13 & 41.19 & 28.33 & 90.75 & 49.14 & 39.89 & 79.07 & 70.09 & 49.16 \\
\hline 613 & 84.49 & 39.31 & 23.80 & 98.97 & 23.80 & 22.77 & 83.62 & 53.76 & 37.37 \\
\hline 614 & 88.04 & 34.43 & 22.47 & 98.17 & 28.03 & 26.20 & 86.43 & 52.81 & 39.24 \\
\hline 615 & 86.87 & 37.93 & 24.80 & 98.09 & 40.19 & 38.27 & 85.21 & 62.87 & 48.08 \\
\hline 616 & 88.42 & 38.11 & 26.54 & 93.55 & 34.47 & 28.02 & 82.72 & 59.45 & 42.16 \\
\hline 617 & 86.68 & 41.10 & 27.78 & 95.97 & 41.00 & 36.97 & 83.19 & 65.25 & 48.43 \\
\hline 618 & 90.36 & 31.58 & 21.94 & 98.36 & 30.86 & 29.22 & 88.88 & 52.69 & 41.57 \\
\hline 619 & 84.55 & 51.55 & 36.09 & 99.30 & 19.74 & 19.05 & 83.96 & 61.11 & 45.07 \\
\hline 620 & 87.20 & 33.15 & 20.35 & 97.86 & 29.00 & 26.86 & 85.33 & 52.54 & 37.87 \\
\hline 621 & 89.36 & 31.83 & 21.19 & 92.31 & 40.85 & 33.16 & 82.49 & 59.68 & 42.17 \\
\hline 622 & 82.11 & 46.67 & 28.78 & 99.91 & 2.57 & 2.49 & 82.04 & 48.04 & 30.08 \\
\hline 623 & 88.55 & 34.89 & 23.44 & 92.89 & 40.87 & 33.76 & 82.25 & 61.50 & 43.76 \\
\hline 624 & 86.03 & 36.19 & 22.23 & 96.30 & 38.95 & 35.25 & 82.85 & 61.04 & 43.90 \\
\hline 625 & 88.78 & 44.74 & 33.52 & 90.65 & 41.66 & 32.31 & 80.47 & 67.76 & 48.24 \\
\hline 626 & 83.47 & 40.61 & 24.08 & 99.43 & 15.49 & 14.92 & 83.00 & 49.81 & 32.80 \\
\hline 627 & 86.36 & 41.77 & 28.13 & 92.77 & 44.91 & 37.68 & 80.12 & 67.92 & 48.04 \\
\hline
\end{tabular}

Table ILL-14 


\section{Combined Microcel/MGS Tests Results - Illinois \#6 Pyritic Sulfur Performance}

\begin{tabular}{|c|c|c|c|c|c|c|c|c|c|}
\hline \multirow[b]{2}{*}{$\begin{array}{c}\text { Test } \\
\text { Number }\end{array}$} & \multicolumn{3}{|c|}{ Microcel } & \multicolumn{3}{|c|}{ MGS } & \multicolumn{3}{|c|}{ Combined Microcel/HGS } \\
\hline & $\begin{array}{c}\text { Comb. } \\
\text { Recovery } \\
\% \\
\end{array}$ & $\begin{array}{c}\text { Pyritic S } \\
\text { Rejection } \\
\% \\
\end{array}$ & $\begin{array}{c}\text { Pyritic S } \\
\text { Separation } \\
\text { Eficiciency } \\
\% \\
\end{array}$ & $\begin{array}{c}\text { Comb. } \\
\text { Recovery } \\
\% \\
\end{array}$ & $\begin{array}{c}\text { Pyritic S } \\
\text { Rejection } \\
\% \\
\end{array}$ & $\begin{array}{c}\text { Pyritic } \mathbf{S} \\
\text { Separation } \\
\text { Efficiency } \\
\% \\
\end{array}$ & $\begin{array}{c}\text { Comb. } \\
\text { Recovery } \\
\% \\
\end{array}$ & $\begin{array}{c}\text { Pyritic S } \\
\text { Rejection } \\
\% \\
\end{array}$ & $\begin{array}{c}\text { Pyritic S } \\
\text { Separation } \\
\text { Efficiency } \\
\% \\
\end{array}$ \\
\hline & & & & & & & & & \\
\hline 601 & 87.70 & 54.14 & 41.84 & 98.48 & 43.01 & 41.49 & 86.37 & 73.86 & 60.24 \\
\hline 602 & 88.13 & 49.12 & 37.25 & 98.60 & 52.09 & 50.69 & 86.89 & 75.63 & 62.52 \\
\hline 603 & 89.18 & 48.20 & 37.39 & 98.60 & 51.78 & 50.38 & 87.94 & 75.02 & 62.96 \\
\hline 604 & 80.51 & 54.50 & 35.01 & 99.03 & 45.87 & 44.90 & 79.73 & 75.37 & 55.10 \\
\hline 605 & 88.40 & 49.40 & 37.80 & 99.17 & 40.46 & 39.63 & 87.67 & 69.87 & 57.54 \\
\hline 606 & 87.31 & 50.21 & 37.52 & 97.76 & 69.97 & 67.73 & 85.35 & 85.05 & 70.40 \\
\hline 607 & 89.16 & 50.82 & 39.97 & 99.60 & 23.91 & 23.51 & 88.80 & 62.58 & 51.38 \\
\hline 608 & 90.60 & 41.47 & 32.07 & 98.89 & 48.98 & 47.87 & 89.59 & 70.14 & 59.73 \\
\hline 609 & 90.21 & 51.11 & 41.32 & 90.02 & 70.66 & 60.68 & 81.21 & 85.66 & 66.87 \\
\hline 610 & 89.95 & 43.81 & 33.76 & 99.19 & 39.28 & 38.48 & 89.23 & 65.88 & 55.11 \\
\hline 611 & 88.72 & 43.41 & 32.13 & 93.44 & 71.02 & 64.46 & 82.90 & 83.60 & 66.50 \\
\hline 612 & 87.13 & 41.44 & 28.57 & 90.75 & 77.54 & 68.29 & 79.07 & 86.85 & 65.92 \\
\hline 613 & 84.49 & 52.68 & 37.16 & 98.97 & 49.14 & 48.12 & 83.62 & 75.93 & 59.55 \\
\hline 614 & 88.04 & 52.57 & 40.61 & 98.17 & 54.44 & 52.61 & 86.43 & 78.39 & 64.82 \\
\hline 615 & 86.87 & 50.28 & 37.16 & 98.09 & 63.51 & 61.59 & 85.21 & 81.86 & 67.07 \\
\hline 616 & 88.42 & 50.01 & 38.44 & 93.55 & 64.16 & 57.71 & 82.72 & 82.08 & 64.80 \\
\hline 617 & 86.68 & 54.92 & 41.60 & 95.97 & 64.58 & 60.55 & 83.19 & 84.03 & 67.22 \\
\hline 618 & 90.36 & 44.78 & 35.15 & 98.36 & 51.51 & 49.87 & 88.88 & 73.23 & 62.11 \\
\hline 619 & 84.55 & 61.19 & 45.74 & 99.30 & 34.32 & 33.63 & 83.96 & 74.51 & 58.47 \\
\hline 620 & 87.20 & 47.04 & 34.24 & 97.86 & 60.75 & 58.61 & 85.33 & 79.21 & 64.55 \\
\hline 621 & 89.36 & 42.71 & 32.07 & 92.31 & 70.04 & 62.35 & 82.49 & 82.83 & 65.32 \\
\hline 622 & 82.11 & 65.47 & 47.58 & 99.91 & 7.64 & 7.55 & 82.04 & 68.11 & 50.15 \\
\hline 623 & 88.55 & 46.06 & 34.62 & 92.89 & 68.45 & 61.33 & 82.25 & 82.98 & 65.24 \\
\hline 624 & 86.03 & 50.13 & 36.16 & 96.30 & 70.43 & 66.74 & 82.85 & 85.25 & 68.11 \\
\hline 625 & 88.78 & 57.94 & 46.72 & 90.65 & 70.10 & 60.75 & 80.47 & 87.43 & 67.90 \\
\hline 626 & 83.47 & 54.94 & 38.41 & 99.43 & 35.15 & 34.58 & 83.00 & 70.78 & 53.77 \\
\hline 627 & 86.36 & 48.94 & 35.31 & 92.77 & 73.64 & 66.41 & 80.12 & 86.54 & 66.66 \\
\hline
\end{tabular}

Table ILL-15 


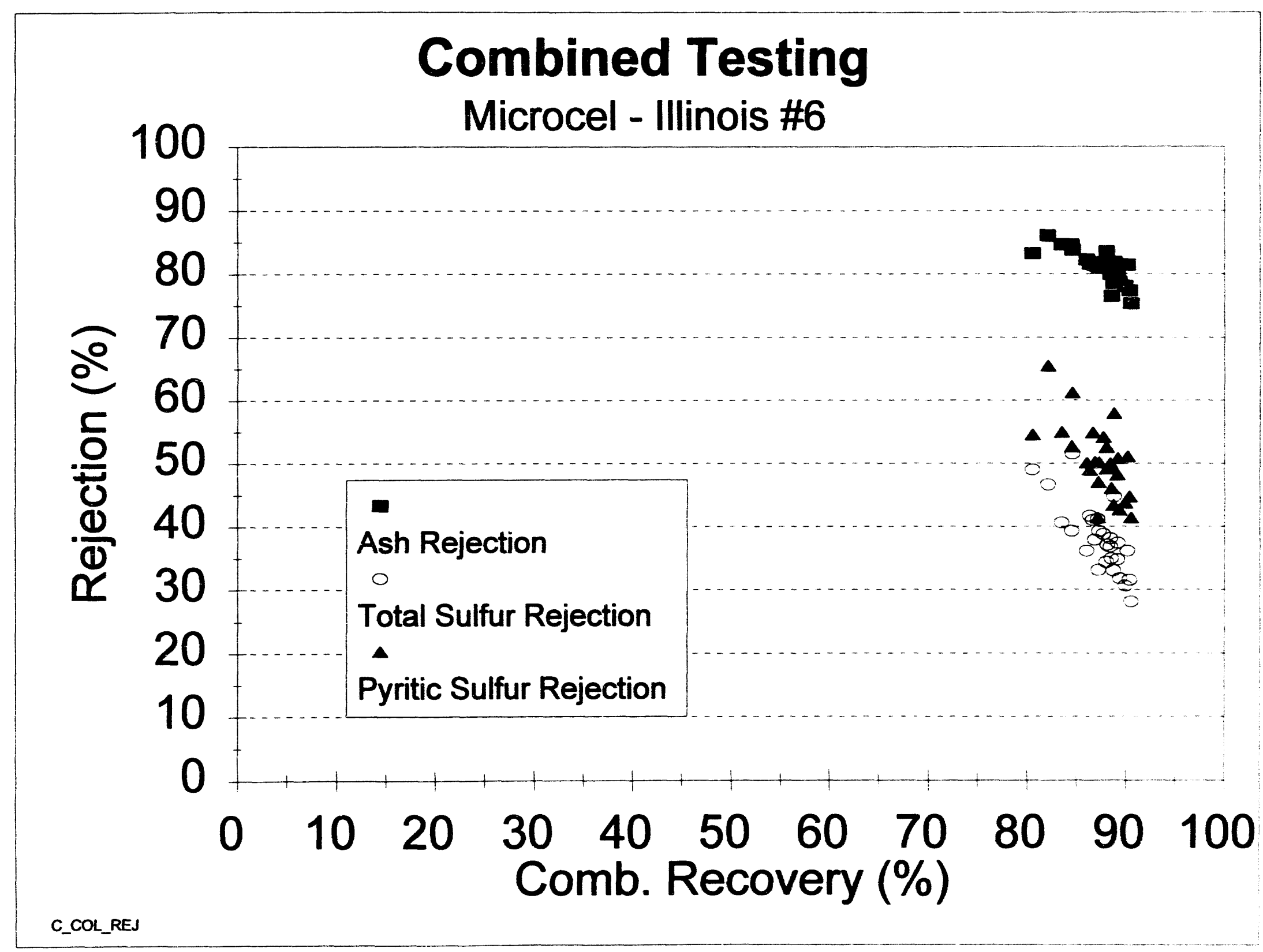

Figure ILL-3 
(\%) Кләлоэәу 'quо

$\begin{array}{lllllllllll}00 L & 06 & 08 & 0 L & 09 & 0 S & 0 t & 0 \varepsilon & 0 Z & \text { OL } & 0\end{array}$

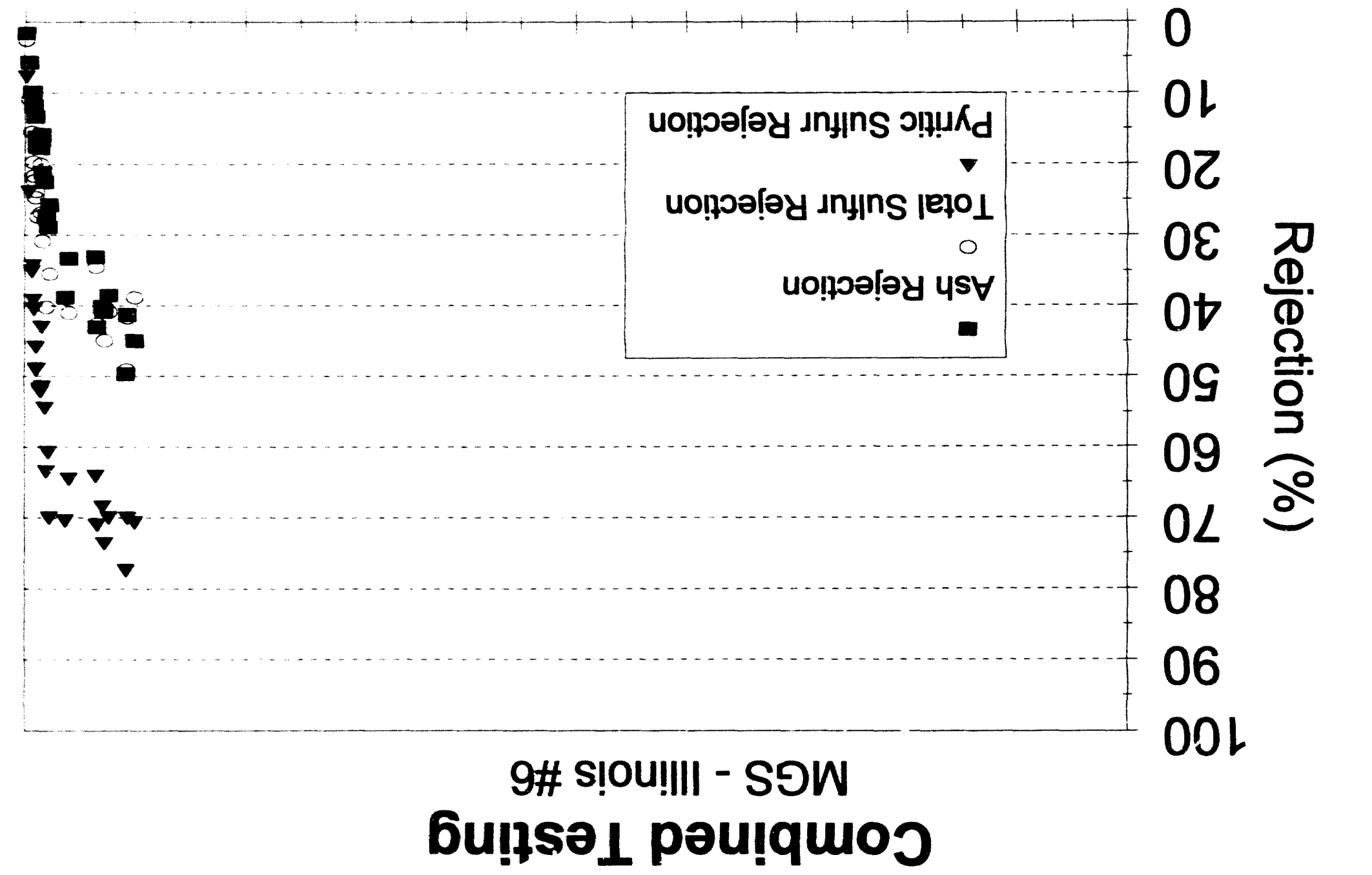




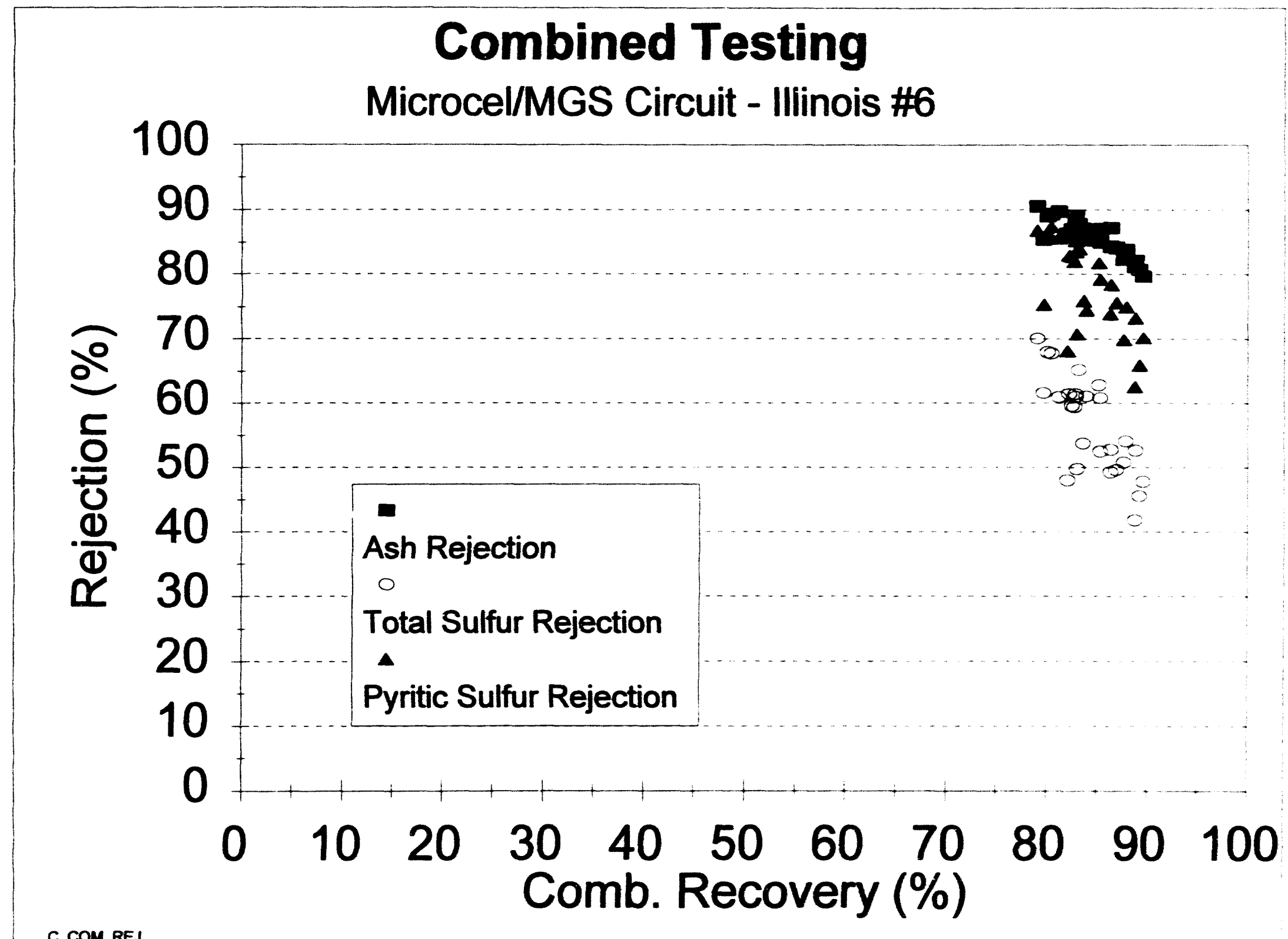

Figure ILL-5 


\section{Microcel/MGS Long Duration Testing - Illinois \#6}

\section{Microcel}

Feed Rate (lbs/ton):
Wash Water (Ipm):

600

40.1

Percent Sollds (w/w):

$11-12$

Grind Size (d80 (microns)):

200

Air Flow Rate (SCFM):

8.89

Diesel dosage (lbs/ton):

Frother Dosage $(\mathrm{ml} / \mathrm{min})$ :

\section{MGS}

Feed Rate (lbs/hr):

Drum Speed (rpm):

340

Wash water (lpm):

283

Percent Solids (w/w):

0.49

Shake Amplitude (mm):

Shake Frequency (cps):

$23-24$

Tilt Angle (degrees): 
Combined Microcel/mGS Tests Results - Illinois \#6

Long Duration Tests - Ash

\begin{tabular}{|c|c|c|c|c|c|c|c|c|c|c|c|c|}
\hline \multirow[b]{2}{*}{$\begin{array}{l}\text { Time } \\
\text { hours }\end{array}$} & \multicolumn{6}{|c|}{ Dicrocel } & \multicolumn{6}{|c|}{ MGS } \\
\hline & $\begin{array}{l}\text { Desoured } \\
\text { microcel } \\
\text { Feed } \\
\text { Ash } \\
\%\end{array}$ & $\begin{array}{l}\text { Adjusted } \\
\text { Microcel } \\
\text { Feed } \\
\text { Ash } \\
\%\end{array}$ & $\begin{array}{l}\text { Messured } \\
\text { microcel } \\
\text { Product } \\
\text { Ash } \\
\text { \% }\end{array}$ & $\begin{array}{l}\text { Adjuated } \\
\text { microcel } \\
\text { Product } \\
\text { Aah } \\
\%\end{array}$ & $\begin{array}{l}\text { Moasured } \\
\text { microcel } \\
\text { Rojoct } \\
\text { Ash } \\
\text { \% }\end{array}$ & $\begin{array}{l}\text { Adjustiod } \\
\text { microcel } \\
\text { Rojoct } \\
\text { Ash } \\
\%\end{array}$ & $\begin{array}{l}\text { mosecured } \\
\text { Hos } \\
\text { Feed } \\
\text { Ash } \\
\%\end{array}$ & $\begin{array}{l}\text { Adjusted } \\
\text { mos } \\
\text { Feed } \\
\text { Ash } \\
\text { \% }\end{array}$ & $\begin{array}{l}\text { Coacured } \\
\text { mos } \\
\text { Lights } \\
\text { Ash } \\
\text { \% }\end{array}$ & $\begin{array}{c}\text { Adjueted } \\
\text { wos } \\
\text { Lights } \\
\text { Ash } \\
\%\end{array}$ & $\begin{array}{l}\text { moasured } \\
\text { mos } \\
\text { Heavies } \\
\text { Ash } \\
\%\end{array}$ & $\begin{array}{l}\text { Adjusted } \\
\text { Mas } \\
\text { Heavies } \\
\text { Ash } \\
\text { \% }\end{array}$ \\
\hline & & & & & & & & & & & & \\
\hline$\overline{1}$ & 35.06 & 35.05 & 10.17 & 9.84 & 77.21 & 77.24 & 9.51 & 9.84 & 7.50 & 7.47 & 45.75 & 45.69 \\
\hline 2 & 27.66 & 27.59 & 8.31 & 8.79 & 75.67 & 75.81 & 9.51 & 8.79 & 7.18 & 7.23 & 46.22 & 46.31 \\
\hline$\frac{5}{3}$ & 29.49 & 29.57 & 8.33 & 8.38 & 72.54 & 72.38 & 8.41 & 8.38 & 6.97 & 6.96 & 49.42 & 49.40 \\
\hline$\overline{4}$ & 28.41 & 28.53 & 8.03 & 8.14 & 72.42 & 72.16 & 8.25 & 8.14 & 6.85 & 6.85 & 51.29 & 51.28 \\
\hline 5 & 25.53 & 25.74 & 7.79 & 7.94 & 71.45 & 70.99 & 8.18 & 7.94 & 6.69 & 6.72 & 54.51 & 54.57 \\
\hline
\end{tabular}

\begin{tabular}{|c|c|c|c|c|c|c|}
\hline $\begin{array}{l}\text { Thime } \\
\text { hours }\end{array}$ & $\begin{array}{l}\text { Masurnod } \\
\text { Meroced } \\
\text { Ash } \\
\text { Yoid } \\
\% \\
\end{array}$ & $\begin{array}{l}\text { Acipuedied } \\
\text { meroed } \\
\text { Ash } \\
\text { Yaid } \\
\% \\
\end{array}$ & $\begin{array}{c}\text { ass } \\
\text { Ash } \\
\text { Yoid } \\
\% \\
\end{array}$ & $\begin{array}{l}\text { Ach } \\
\text { Yold } \\
\text { \% }\end{array}$ & $\begin{array}{l}\text { Combined } \\
\text { Coh } \\
\text { Yoid } \\
\%\end{array}$ & 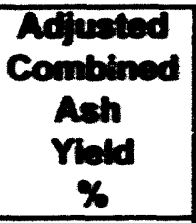 \\
\hline 1 & 62.87 & 62.60 & 94.75 & 93.80 & 59.57 & 58.71 \\
\hline 2 & 71.27 & 71.95 & 94.03 & 96.01 & 67.02 & 69.08 \\
\hline 3 & 67.05 & 66.89 & 96.61 & 96.65 & 64.77 & 64.65 \\
\hline 4 & 68.35 & 68.15 & 96.85 & 97.10 & 66.20 & 66.17 \\
\hline 5 & 72.13 & 71.77 & 96.88 & 97.45 & 69.89 & 69.94 \\
\hline
\end{tabular}




\section{Combined MicrocelMMGS Tests Results - IIlinois \#6}

\section{Long Duration Tests - Total Sulfur}

\begin{tabular}{|c|c|c|c|c|c|c|c|c|c|c|c|c|}
\hline \multirow[b]{2}{*}{$\begin{array}{l}\text { Time } \\
\text { hours }\end{array}$} & \multicolumn{6}{|c|}{ Dicrocel } & \multicolumn{6}{|c|}{ MGS } \\
\hline & $\begin{array}{l}\text { Claseured } \\
\text { Microcel } \\
\text { Feed } \\
\text { T. Sulfur } \\
\%\end{array}$ & $\begin{array}{l}\text { Adjusted } \\
\text { Microcel } \\
\text { Feed } \\
\text { T. Sulfur } \\
\text { \% }\end{array}$ & $\begin{array}{l}\text { Maseured } \\
\text { merocel } \\
\text { Product } \\
\text { T. Sulfur } \\
\%\end{array}$ & $\begin{array}{l}\text { Adjueted } \\
\text { merocel } \\
\text { Product } \\
\text { T. Sulfur } \\
\text { \% }\end{array}$ & $\begin{array}{l}\text { Tassured } \\
\text { emcroced } \\
\text { Rejoct } \\
\text { T. Sulfur } \\
\text { \% }\end{array}$ & $\begin{array}{l}\text { Adjucted } \\
\text { Merocel } \\
\text { Reject } \\
\text { T. Sulfur } \\
\text { \% }\end{array}$ & $\begin{array}{l}\text { Cosened } \\
\text { mos } \\
\text { Feed } \\
\text { T. Sulfur } \\
\text { x } \\
\end{array}$ & $\begin{array}{l}\text { Acjuetod } \\
\text { nes } \\
\text { Feed } \\
\text { T. Sulfur } \\
\% \\
\end{array}$ & \begin{tabular}{c|} 
Crasured \\
mos \\
Lights \\
T. Sulfur \\
\% \\
\end{tabular} & $\begin{array}{c}\text { Adjusted } \\
\text { mes } \\
\text { Lights } \\
\text { T. Suthur } \\
\text { \% }\end{array}$ & $\begin{array}{c}\text { masured } \\
\text { nos } \\
\text { Heavies } \\
\text { T. Sulfur } \\
\% \\
\end{array}$ & 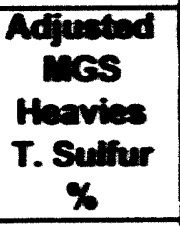 \\
\hline & & & & & & & & & & & & \\
\hline 1 & 3.98 & 4.30 & 3.87 & 4.15 & 4.73 & 4.56 & 3.78 & 4.15 & 2.81 & 3.02 & 20.60 & 21.33 \\
\hline 2 & 3.14 & 3.56 & 3.16 & 3.31 & 4.42 & 4.19 & 3.78 & 3.31 & 2.65 & 2.56 & 21.70 & 21.45 \\
\hline 3 & 3.33 & 3.40 & 3.12 & 3.22 & 3.79 & 3.76 & 3.40 & 3.22 & 2.48 & 2.49 & 24.10 & 24.14 \\
\hline$\overline{4}$ & 3.29 & 3.42 & 3.14 & 3.15 & 4.04 & 3.98 & 3.26 & 3.15 & 2.42 & 2.43 & 27.30 & 27.33 \\
\hline & 3.00 & 3.38 & 2.76 & 2.81 & 5.12 & 4.81 & 2.98 & 2.81 & 2.23 & 2.15 & 28.60 & 28.24 \\
\hline
\end{tabular}




\section{Combined Microcel/MGS Tests Results - Illinois \#6}

Long Duration Teats - Pyritic Sulfur

\begin{tabular}{|c|c|c|c|c|c|c|c|c|c|c|c|c|}
\hline \multirow[b]{2}{*}{$\begin{array}{l}\text { Time } \\
\text { hours }\end{array}$} & \multicolumn{6}{|c|}{ Dicrocel } & \multicolumn{6}{|c|}{ MGS } \\
\hline & $\begin{array}{c}\text { Thassured } \\
\text { Microcel } \\
\text { Feed } \\
\text { Pyrtic S } \\
\text { \% }\end{array}$ & $\begin{array}{c}\text { Adjusted } \\
\text { Mcrocel } \\
\text { Feed } \\
\text { Pyritic } \mathbf{5} \\
\text { \% }\end{array}$ & $\begin{array}{l}\text { Measured } \\
\text { Microcel } \\
\text { Product } \\
\text { Pyritic S } \\
\%\end{array}$ & $\begin{array}{c}\text { Adjustod } \\
\text { Microcel } \\
\text { Product } \\
\text { Pyritic S } \\
\%\end{array}$ & \begin{tabular}{c|} 
Mosasured \\
Microcel \\
Reject \\
Pyritic S \\
$\%$
\end{tabular} & $\begin{array}{c}\text { Adjueted } \\
\text { Menocel } \\
\text { Reject } \\
\text { Pyritic } \mathbf{S} \\
\text { \% }\end{array}$ & $\begin{array}{l}\text { Coasured } \\
\text { mGS } \\
\text { Feed } \\
\text { Pyritic S } \\
\%\end{array}$ & $\begin{array}{l}\text { Adjuested } \\
\text { mos } \\
\text { Feed } \\
\text { Pyritic S } \\
\%\end{array}$ & $\begin{array}{l}\text { messured } \\
\text { mos } \\
\text { Lights } \\
\text { Pyritic S } \\
\text { \% }\end{array}$ & $\begin{array}{l}\text { Adjusted } \\
\text { mas } \\
\text { Lights } \\
\text { Pyritic } 5 \\
\%\end{array}$ & $\begin{array}{l}\text { moasured } \\
\text { mos } \\
\text { Heavies } \\
\text { Pyritic S } \\
\text { \% }\end{array}$ & $\begin{array}{c}\text { Adjusted } \\
\text { mos } \\
\text { Heavies } \\
\text { Pyritic S } \\
\%\end{array}$ \\
\hline & & & & & & & & & & & & \\
\hline 1 & 2.28 & 2.29 & 1.66 & 1.67 & 3.12 & 3.16 & 1.52 & 1.67 & 0.69 & 0.66 & 18.41 & 17.02 \\
\hline 2 & 1.56 & 1.85 & 1.25 & 1.37 & 3.53 & 3.11 & 1.52 & 1.37 & 0.69 & 0.65 & 20.20 & 18.67 \\
\hline & 1.96 & 1.89 & 1.38 & 1.37 & 2.89 & 2.94 & 1.37 & 1.37 & 0.67 & 0.68 & 21.16 & 21.41 \\
\hline & 1.58 & 1.70 & 1.63 & 1.30 & 2.66 & 2.55 & 1.16 & 1.3 & 0.64 & 0.63 & 23.90 & 23.64 \\
\hline & 1.40 & 1.73 & 1.13 & 1.15 & 3.92 & 3.19 & 1.21 & 1.15 & 0.61 & 0.57 & 24.73 & 23.22 \\
\hline
\end{tabular}


Combined Microcel/MGS Tests Results - Illinois \#6

Long Duration Tests - Ash Performance

\begin{tabular}{|c|c|c|c|c|c|c|c|c|c|}
\hline \multirow[b]{2}{*}{$\begin{array}{l}\text { Time } \\
\text { hours }\end{array}$} & \multicolumn{3}{|c|}{ Microcel } & \multicolumn{3}{|c|}{ MGS } & \multicolumn{3}{|c|}{ Combined NicrocelWGS } \\
\hline & $\begin{array}{c}\text { Comb. } \\
\text { Recovery } \\
\% \\
\end{array}$ & $\begin{array}{c}\text { Ash } \\
\text { Rejection } \\
\%\end{array}$ & $\begin{array}{c}\text { Ash } \\
\text { Separation } \\
\text { Eficiency } \\
\%\end{array}$ & $\begin{array}{c}\text { Comb. } \\
\text { Recovery } \\
\%\end{array}$ & $\begin{array}{c}\text { Ash } \\
\text { Rejection } \\
\% \\
\end{array}$ & $\begin{array}{c}\text { Ash } \\
\text { Separation } \\
\text { Eficiency } \\
\%\end{array}$ & $\begin{array}{c}\text { Comb. } \\
\text { Recovery } \\
\% \\
\end{array}$ & $\begin{array}{c}\text { Ash } \\
\text { Rejection } \\
\% \\
\end{array}$ & $\begin{array}{c}\text { Ash } \\
\text { Separation } \\
\text { Eficiency } \\
\% \\
\end{array}$ \\
\hline & & & & & & & & & \\
\hline 1 & 86.89 & 82.43 & 69.32 & 96.26 & 28.79 & 25.06 & 83.65 & 87.49 & 71.13 \\
\hline$\overline{2}$ & 90.63 & 77.08 & 67.71 & 97.65 & 21.03 & 18.68 & 88.50 & 81.90 & 70.40 \\
\hline$\overline{3}$ & 87.02 & 81.04 & 68.06 & 98.15 & 19.72 & 17.88 & 85.41 & 84.78 & 70.19 \\
\hline$\overline{4}$ & 87.59 & 80.56 & 68.15 & 98.46 & 18.29 & 16.75 & 86.24 & 84.11 & 70.36 \\
\hline 5 & 88.97 & 77.86 & 66.83 & 98.74 & 17.52 & 16.26 & 87.85 & 81.74 & 69.59 \\
\hline
\end{tabular}




\section{Combined Microcel/MGS Tests Results - Illinois \#6}

\section{Long Duration Tests - Total Sulfur Performance}

\begin{tabular}{|r||c|c|c|r|r|r|r|r|r|}
\hline \multirow{2}{*}{$\begin{array}{c}\text { Time } \\
\text { hours }\end{array}$} & \multicolumn{3}{|c|}{ Microcel } & \multicolumn{3}{c|}{ MGS } & \multicolumn{3}{c|}{ Combined Microcel/MGS } \\
\hline Recovery & $\begin{array}{c}\text { T. Sulfur } \\
\text { Rejection }\end{array}$ & $\begin{array}{c}\text { T. Sulfur } \\
\text { Separation } \\
\text { Efficiency } \\
\%\end{array}$ & $\begin{array}{c}\text { Comb. } \\
\text { Recovery }\end{array}$ & $\begin{array}{c}\text { T. Sulfur } \\
\text { Rejection }\end{array}$ & $\begin{array}{c}\text { T. Sulfur } \\
\text { Separation } \\
\text { Efficiency } \\
\%\end{array}$ & $\begin{array}{c}\text { Comb. } \\
\text { Recovery }\end{array}$ & $\begin{array}{c}\text { T. Sulfur } \\
\text { Rejection } \\
\%\end{array}$ & $\begin{array}{c}\text { T. Sulfur } \\
\text { Separation } \\
\text { Efficiency } \\
\%\end{array}$ \\
\hline 1 & & & & & & & & & \\
\hline 2 & 86.89 & 39.59 & 26.48 & 96.26 & 31.74 & 28.01 & 83.65 & 58.76 & 42.41 \\
\hline 3 & 80.63 & 33.10 & 23.73 & 97.65 & 25.75 & 23.40 & 88.50 & 50.33 & 38.83 \\
\hline 4 & 87.02 & 36.65 & 23.67 & 98.15 & 25.26 & 23.41 & 85.41 & 52.65 & 38.06 \\
\hline 5 & 88.97 & 37.23 & 24.82 & 98.46 & 25.10 & 23.56 & 86.24 & 52.98 & 39.23 \\
\hline
\end{tabular}


Combined Microcel/MGS Tests Results - Illinois \#6

Long Duration Tests - Pyritic Sulfur Performance

\begin{tabular}{|c|c|c|c|c|c|c|c|c|c|}
\hline \multirow[b]{2}{*}{$\begin{array}{l}\text { Time } \\
\text { hours }\end{array}$} & \multicolumn{3}{|c|}{ Microcel } & \multicolumn{3}{|c|}{ MGS } & \multicolumn{3}{|c|}{ Combined Microcel/MGS } \\
\hline & $\begin{array}{c}\text { Comb. } \\
\text { Recovery } \\
\% \\
\end{array}$ & $\begin{array}{c}\text { Pyritic S } \\
\text { Rejection } \\
\% \\
\end{array}$ & $\begin{array}{c}\text { Pyritic S } \\
\text { Separation } \\
\text { Efficiency } \\
\% \\
\end{array}$ & $\begin{array}{c}\text { Comb. } \\
\text { Recovery } \\
\% \\
\end{array}$ & $\begin{array}{c}\text { Pyritic S } \\
\text { Rejection } \\
\% \\
\end{array}$ & $\begin{array}{c}\text { Pyritic S } \\
\text { Separation } \\
\text { Efficiency } \\
\% \\
\end{array}$ & $\begin{array}{c}\text { Comb. } \\
\text { Recovery } \\
\% \\
\end{array}$ & $\begin{array}{c}\text { Pyritic S } \\
\text { Rejection } \\
\% \\
\end{array}$ & $\begin{array}{c}\text { Pyritic S } \\
\text { Separation } \\
\text { Efficiency } \\
\% \\
\end{array}$ \\
\hline & & & & & & & & & \\
\hline 1 & 86.89 & 54.35 & 41.24 & 96.26 & 62.93 & 59.19 & 83.65 & 83.08 & 66.73 \\
\hline 2 & 90.63 & 46.72 & 37.35 & 97.65 & 54.45 & 52.10 & 88.50 & 75.73 & 64.23 \\
\hline 3 & 87.02 & 51.51 & 38.53 & 98.15 & 52.03 & 50.18 & 85.41 & 76.74 & 62.15 \\
\hline 4 & 87.59 & 47.88 & 35.48 & 98.46 & 52.95 & 51.41 & 86.24 & 75.48 & 61.72 \\
\hline & 88.97 & 52.29 & 41.26 & 98.74 & 51.70 & 50.44 & 87.85 & 76.96 & 64.81 \\
\hline
\end{tabular}




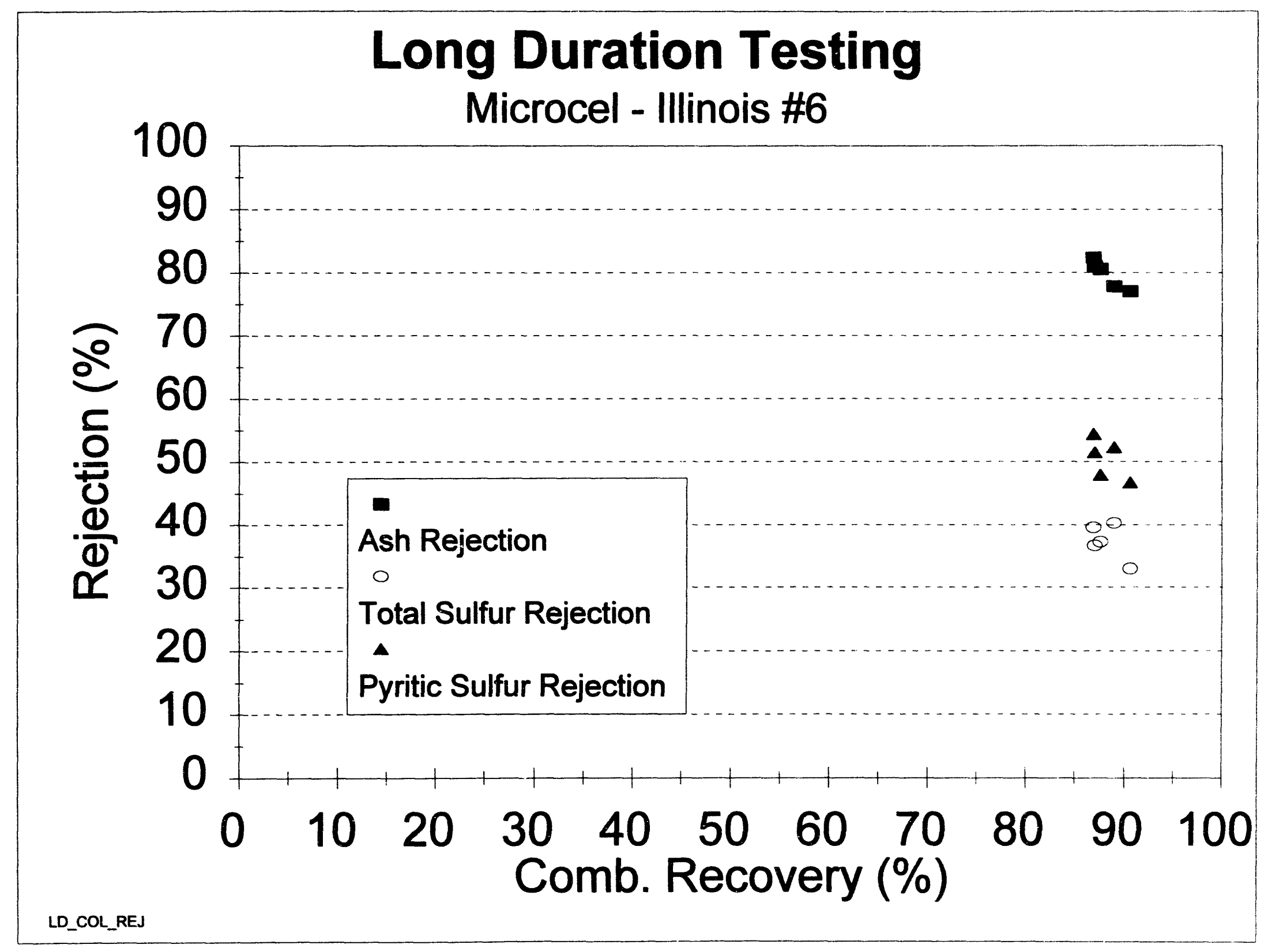

Figure ILL-6 


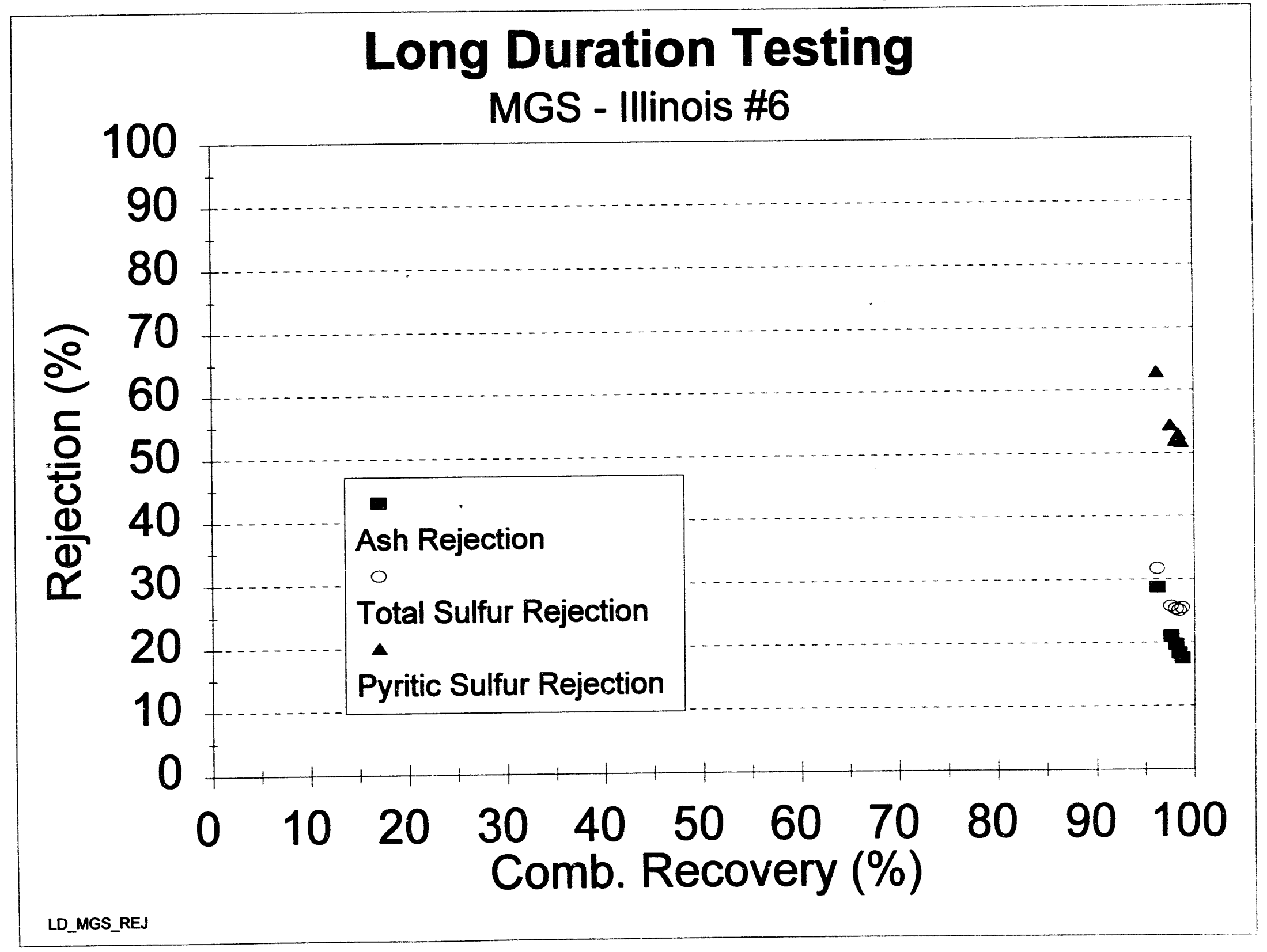

Figure ILL-7 


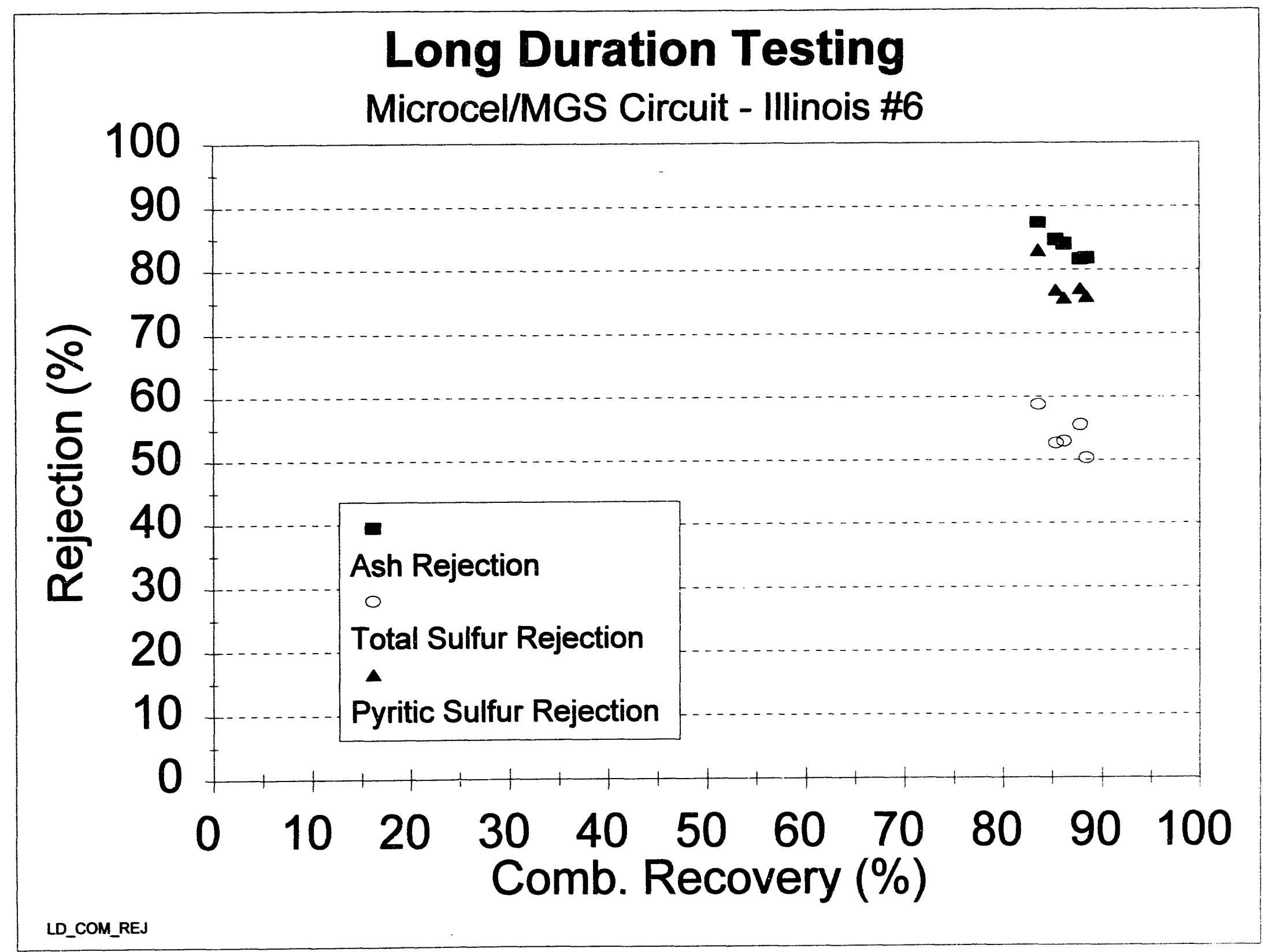

Figure ILL-8 

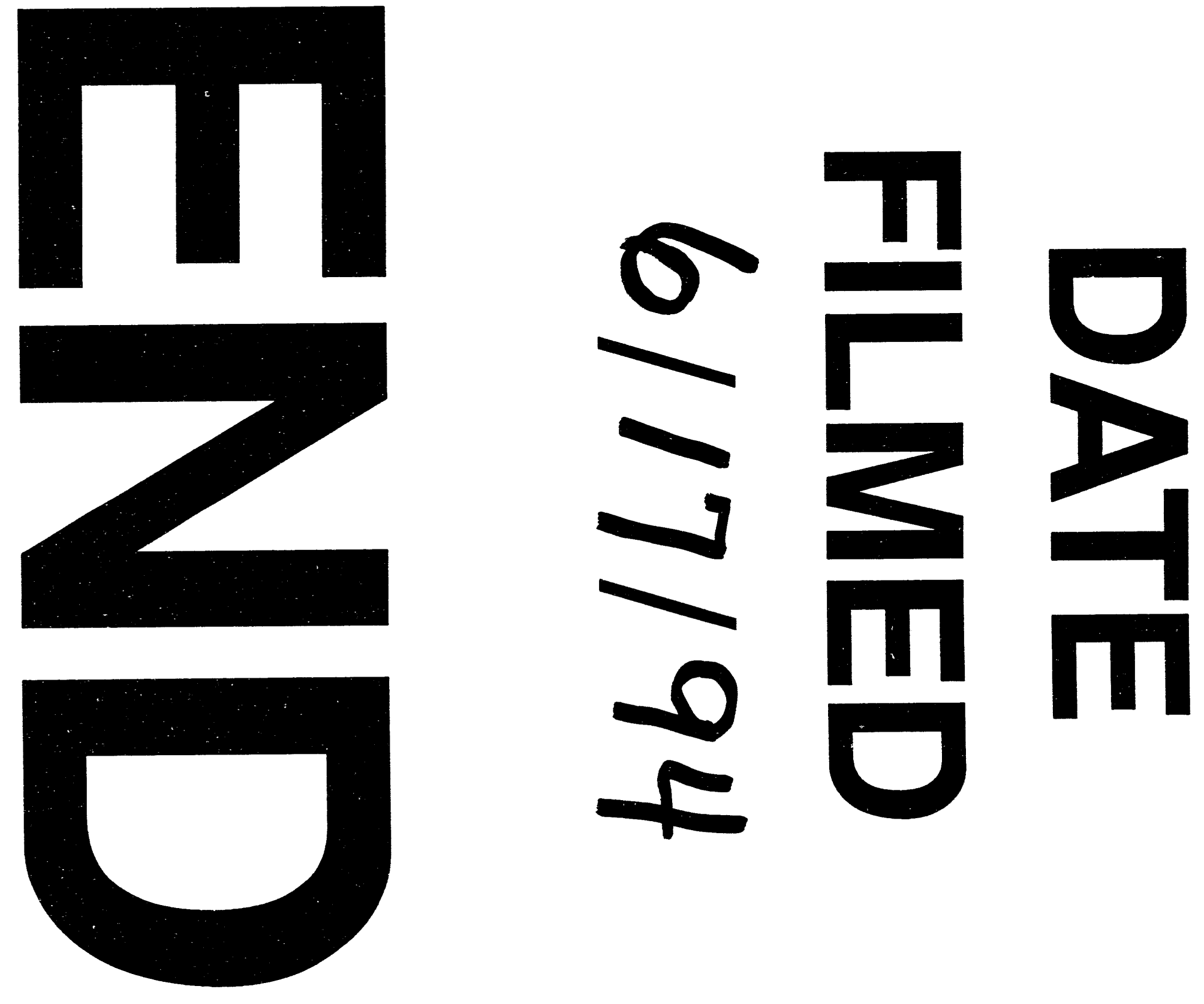

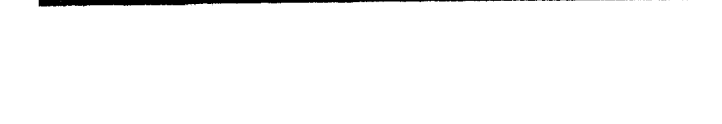
-...

$$
\underline{-12}
$$

